TECHNICAL NOTE R-213

BOEING DOCUMENT DI-82-0568

\title{
DIRECTIONAL CHARACTERISTICS OF LUNAR THERMAL EMISSION
}

\author{
By \\ C. G. Montgomery \\ J. M. Saari* \\ R. W. Shorthill* \\ N. F. Six, Jr. \\ Prepared For
}

SPACE THERMODYNAMICS BRANCH

RESEARCH PROJECTS LABORATORY

GEORGE C. MARSHALL SPACE FLIGHT CENTER

\author{
Prepared By \\ RESEARCH LABORATORIES \\ BROWN ENGINEERING COMPANY, INC. \\ HUNTSVILLE, ALABAMA 35805 \\ Contract No. NAS8-20166 \\ in cooperation with \\ * BOEING SCIENTIFIC RESEARCH LABORATORIES \\ GEO-ASTROPHYSICS LABORATORY \\ SEATTLE, WASHINGTON 98124
}




\section{ABSTRACT}

Preliminary studies of the directional characteristics of lunar thermal emission have been completed using existing infrared lunation data. Brightness temperatures were obtained at every $10^{\circ}$ interval of a thermal latitude and longitude grid system where the subsolar point was defined as the thermal north pole, the antisolar point as the the rmal south pole, and the thermal prime meridian as passing through the apparent disk center. The results are presented in the form of a family of plots for:

1. Brightness temperature versus thermal longitude at constant phase for different thermal latitudes.

2. Brightness temperature versus thermal longitude at constant thermal latitude for different phases.

3. Brightness temperature versus phase at constant latitude for different the rmal longitudes.

For brightness temperature along the thermal meridian the Lambert diffuse surface law $T=T_{0} \cos ^{\frac{1}{4}} \theta$ seems to hold for large phase angles. As the phase decreases a $\cos ^{\frac{1}{6}} \theta$ variation is followed. At all phases, however, there is a deviation from these laws at large distances from the subsolar point.

For phase angles less than about $50^{\circ}$, i.e., when the observer is within $50^{\circ}$ of the Sun line, the brightness temperature is greater than the Lambert temperature. However, the maximum brightness temperature is not generally observed exactly in the direction of the Sun, but rather at an elevation angle less than the Sun. This effect can be qualitatively explained in terms of a flat surface interspersed with hemispherical craters. 
TABLE OF CONTENTS

Page

I. INTRODUCTION . . . . . . . . . . . . . . . . . I

II. PREVIOUS DIRECTIONAL MEASUREMENTS . . . . . . . 1

III. THE INFRARED SCAN DATA OF THE BOEING SCIENTIFIC RESEARCH LABORATORIES . . . . . . . . 3

IV. EXAMPLES OF DATA ALONG THE THERMAL MERIDIAN . 4

V. DEFINITION OF THERMAL COORDINATES. . . . . . . . 5

VI. THERMAL GRID OVERLAYS AND BRIGHTNESS

TEMPERATURE VERSUS THERMAL LONGITUDE -

PHASE CONSTANT . . . . . . . . . . . . . . . . . 5

VII. BRIGHTNESS TEMPERATURE VERSUS THERMAL LONGITUDE - LATITUDE CONSTANT . . . . . . . . . 6

VIII. BRIGHTNESS TEMPERATURE VERSUS PHASE LATITU DE CONSTANT . . . . . . . . . . . . . . . . . 7

IX. FACTORS AFFECTING THE BRIGHT NESS TEMPERATURE VALUES . . . . . . . . . . . . . . . . . . . . 8

X. DISCUSSION . . . . . . . . . . . . . . . . . . . 9

XI. REFERENCES . . . . . . . . . . . . . . . . . 13

TABLES AND FIGURES . . . . . . . . . . . . . . . . . . 15

$\mathrm{v} / \mathrm{vi}$ 


\section{LIST OF FIGURES}

Figure

Page

1 Distribution of Lunar Heat with Angular Distance from the Subsolar Point . . . . . . . . . . . . . . . . . . 19

2 View Looking Down on the North Pole of the Moon. The Sun's radiation is incident from the right, and the observer is on the Sun-Moon line . . . . . . . . . .

3 Progression of the Subsolar Point Around the Earthside Hemisphere of the Moon. The Sun's radiation is incident from the right; $\alpha$ is the phase angle, and the observer is at $0 . \ldots . \ldots$

4 Polar Plot of Lunar Heat from the Subsolar Point as a Function of Phase Angle $\alpha$. . . . . . . . . . . . . . 21

5 Isothermal Contours for $-2^{\circ} 16^{\prime}$ Phase Angle with a Standard Orthographic Grid Overlay . . . . . . . . . .

6 Scan Paths Examined to Determine the Angular Distribution of Brightness Temperature . . . . . . . .

7 Variation of Brightness Temperature $\mathrm{T}$ with $\theta$, Angular Distance from the Subsolar Point. Data for the the rmal meridian.

8 USAF Lunar Earthside Mosaic (LEM-1) Produced by the Aeronautical Chart and Information Center, St. Louis, Missouri . . . . . . . . . . . . . . . . .

9 Thermal Coordinate Grid for Phase Angle $-113^{\circ} 20^{\prime}$. . .

10 Brightness Temperature Versus Thermal Longitude at Phase $-113^{\circ} 20^{\prime}$. . . . . . . . . . . . . .

11 Thermal Coordinate Grid for Phase Angle -102 $15^{\prime}$. . .

12 Brightness Temperature Versus Thermal Longitude at Phase $-102^{\circ} 15^{\prime}$. . . . . . . . . . . . . . . . . .

13 Thermal Coordinate Grid for Phase Angle - $91^{\circ} 52^{\prime}$. . . 30

14 Brightness Temperature Versus Thermal Longitude at Phase $-91^{\circ} 52^{\prime}$. 
LIST OF FIGURES (Continued)

Figure

Page

15 Thermal Coordinate Grid for Phase Angle $-88^{\circ} 41^{\prime}$. . . . 32

16 Brightness Temperature Versus Thermal Longitude at Phase $-88^{\circ} 41^{\prime}$. . . . . . . . . . . . . . . 33

17 Thermal Coordinate Grid for Phase Angle $-65^{\circ} 29^{\prime}$. . . . 34

18 Brightness Temperature Versus Thermal Longitude at $-65^{\circ} 29^{\prime}$. . . . . . . . . . . . . . . . 35

19 Thermal Coordinate Grid for Phase Angle - $39^{\circ} 52^{\prime}$. . . . 36

20 Brightness Temperature Versus Thermal Longitude at Phase $-39^{\circ} 52^{\prime}$. . . . . . . . . . . . . . 37

21 Thermal Coordinate Grid for Phase Angle -29 $15^{\prime}$. . . . 38

22 Brightness Temperature Versus Thermal Longitude at Phase $-29^{\circ} 15^{\circ}$. . . . . . . . . . . . . . 39

23 Thermal Coordinate Grid for Phase Angle - $15^{\circ} 28^{\prime}$. . . . 40

24 Brightness Temperature Versus Thermal Longitude at Phase $-15^{\circ} 28^{\prime}$. . . . . . . . . . . . . . . 41

25 Thermal Coordinate Grid for Phase Angle $-2^{\circ} 16^{\prime}$. . . . 42

26 Brightness Temperature Versus Thermal Longitude at Phase $-2^{\circ} 16^{\prime}$. . . . . . . . . . . . . . . . 43

27 Thermal Coordinate Grid for Phase Angle $+11^{\circ} 42^{\prime}$. . . . 44

28 Brightness Temperature Versus Thermal Longitude at Phase $+11^{\circ} 42^{\prime}$. . . . . . . . . . . . . . . . . 45

29 Thermal Coordinate Grid for Phase Angle $+25^{\circ} 37^{\prime}$. . . . 46

30 Brightness Temperature Versus Thermal Longitude at Phase $+25^{\circ} 37^{\prime}$. . . . . . . . . . . . . . 47

31 Thermal Coordinate Grid for Phase Angle $+39^{\circ} 51^{\prime}$. . . . 48 


\section{LIST OF FIGURES (Continued)}

Figure

32 Brightness Temperature Versus Thermal Longitude at $+39^{\circ} 51^{\prime}$. . . . . . . . . . . . . . . . . . . . 49

33 Thermal Coordinate Grid for Phase Angle $+49^{\circ} 12^{\prime}$. . . . 50

34 Brightness Temperature Versus Thermal Longitude at Phase $+49^{\circ} 12^{\prime}$. . . . . . . . . . . . . . . 51

35 Thermal Coordinate Grid for Phase Angle $+63^{\circ} 23^{\prime}$. . . . 52

36 Brightness Temperature Versus Thermal Longitude at Phase $+63^{\circ} 23^{\prime}$. . . . . . . . . . . . . . 53

37 Thermal Coordinate Grid for Phase Angle $+76^{\circ} 42^{\prime}$. . . . 54

38 Brightness Temperature Versus Thermal Longitude at Phase $+76^{\circ} 42^{\prime}$. . . . . . . . . . . . . . . . 55

39 Thermal Coordinate Grid for Phase Angle $+90^{\circ} 16^{\prime}$. . . . 56

40 Brightness Temperature Versus Thermal Longitude at Phase $+90^{\circ} 16^{\prime}$. . . . . . . . . . . . . . . 57

41 Thermal Coordinate Grid for Phase Angle $+98^{\circ} 40^{\prime}$. . . . 58

42 Brightness Temperature Versus Thermal Longitude at Phase $+98^{\circ} 40^{\prime}$. . . . . . . . . . . . . . 59

43 Thermal Coordinate Grid for Phase Angle $+123^{\circ} 51^{\prime}$. . . 60

44 Brightness Temperature Versus Thermal Longitude at Phase $+123^{\circ} 51^{\prime}$. . . . . . . . . . . . . . . 61

45 Thermal Coordinate Grid for Phase Angle $+135^{\circ} 40^{\prime}$. . . 62

46 Brightness Temperature Versus Thermal Longitude at Phase $+135^{\circ} 40^{\prime}$. . . . . . . . . . . . . . . . 63

47 Brightness Temperature Versus Thermal Longitude for Negative Phases at Thermal Latitude $80^{\circ}$. . . . . . . . 64

48 Brightness Temperature Versus Thermal Longitude for Negative Phases at Thermal Latitude $70^{\circ}$. . . . . . . 65 


\section{LIST OF FIGURES (Continued)}

Figure

Page

49 Brightness Temperature Versus Thermal Longitude for Negative Phases at Thermal Latitude $60^{\circ}$. . . . . . . . 66

50 Brightness Temperature Versus Thermal Longitude for Negative Phases at Thermal Latitude $50^{\circ}$. . . . . . . . 67

51 Brightness Temperature Versus Thermal Longitude for Negative Phases at Thermal Latitude $40^{\circ}$. . . . . . . . 68

52 Brightness Temperature Versus Thermal Longitude for Negative Phases at Thermal Latitude $30^{\circ}$. . . . . . . . 69

53 Brightness Temperature Versus Thermal Longitude for Negative Phases at Thermal Latitude $20^{\circ}$. . . . . . . 70

54 Brightness Temperature Versus Thermal Longitude for Negative Phases at Thermal Latitude $10^{\circ}$. . . . . . . . 71

55 Brightness Temperature Versus Thermal Longitude for Positive Phases at Thermal Latitude $80^{\circ}$. . . . . . . . 72

56 Brightness Temperature Versus Thermal Longitude for Positive Phases at Thermal Latitude $70^{\circ}$. . . . . . . . 73

57 Brightness Temperature Versus Thermal Longitude for Positive Phases at Thermal Latitude $60^{\circ}$. . . . . . . . 74

58 Brightness Temperature Versus Thermal Longitude for Positive Phases at Thermal Latitude $50^{\circ}$. . . . . . . . 75

59 Brightness Temperature Versus Thermal Longitude for Positive Phases at Thermal Latitude $40^{\circ}$. . . . . . . . 76

60 Brightness Temperature Versus Thermal Longitude for Positive Phases at Thermal Latitude $30^{\circ}$. . . . . . . . 77

61 Brightness Temperature Versus Thermal Longitude for Positive Phases at Thermal Latitude $20^{\circ}$. . . . . . . . 78

62 Brightness Temperature Versus Thermal Longitude for Positive Phases at Thermal Latitude $10^{\circ}$. . . . . . . . 79

63 Brightness Temperature Versus Phase at Thermal Latitude $80^{\circ}$. . . . . . . . . . . . . . . . . . . . . 80 
LIST OF FIGURES (Continued)

Figure

Page

64 Brightness Temperature Versus Phase at Thermal

Latitude $70^{\circ}$. . . . . . . . . . . . . . . . . . 81

65 Brightness Temperature Versus Phase at Thermal

Latitude $60^{\circ}$. . . . . . . . . . . . . . . . . 82

66 Brightness Temperature Versus Phase at Thermal

Latitude $50^{\circ}$. . . . . . . . . . . . . . . . . . . . . 83

67 Brightness Temperature Versus Phase at Thermal Latitude $40^{\circ}$. . . . . . . . . . . . . . . . . . . . 84

68 Brightness Temperature Versus Phase at Thermal

Latitude $30^{\circ}$. . . . . . . . . . . . . . . . . . . . . 85

69 Brightness Temperature Versus Phase at Thermal

Latitude $20^{\circ}$. . . . . . . . . . . . . . . . . . . . . 86

70 Brightness Temperature Versus Phase at Thermal

Latitude $10^{\circ}$. . . . . . . . . . . . . . . . . . 87

71 Variation in Ratio of Shadow to Illuminated Area

Between Two Craters Equal Angular Distances from

the Subsolar Point, as Seen by an Earth-Sensor . . . . . 88

72 Brightness Temperature Versus Phase for $30^{\circ}$

Thermal Longitude Before Full Moon and for $-150^{\circ}$

Thermal Longitude After Full Moon at Several

Thermal Latitudes . . . . . . . . . . . . . . . 89

73 Crater Model of the Lunar Surface . . . . . . . . . 90 


\section{INTRODUCTION}

Extensive measurements have been made of the directional characteristics of the light reflected from the Moon. The observed strong backscattering has been compared with photometric studies in the laboratory of natural and contrived surfaces and with theoretical models, and has contributed significantly to our understanding of the nature of the lunar surface. Directional properties have been noted in the infrared emission from the Moon as well. However, the measurements have been far less extensive so that the interpretation of the results has been limited to qualitative explanations in terms of surface roughness.

The purpose of the work reported here was to obtain from existing data a new set of measurements relating to the directional characteristics of lunar infrared emission. The data are based on measurements resulting from a program at the Boeing Scientific Research Laboratories which consisted of scanning the illuminated lunar disk in the far-infrared and visible wavelengths through a lunation. The effort here has consisted mainly of a reduction of 5000 data points from 19 separate phases in terms of "thermal coordinates" appropriate to the measurements.

The data are applicable to engineering calculations of the heat balance of spacecraft on the lunar surface. Further, theoretical models of surface roughness can be formulated with respect to these data, and compared with measurements made at other wavelengths.

\section{PREVIOUS DIRECTIONAL MEASUREMENTS}

The directionality of infrared emission from the lunar surface has been observed by two methods previously. The first consisted of telescope scans across the lunar disk near Full Moon, i.e., when the phase angle $\alpha$ (Sun-Moon-observer) is close to $0^{\circ}$. Figure 1 shows the results of Pettit and Nicholson (1930) of such a measurement of the lunar heat as 
a function of angular distance $\theta$ from the subsolar point. If the Moon behaved like a Lambert surface, the planetary heat should vary as $\cos \theta$, since the energy received by a unit element depends in this manner on the local zenith angle of the sun (see Fig. 2). This function is shown by the dashed curve in Fig. 1. Actually, the measurements show that values higher than this are observed near the limb. Pettit and Nicholson suggested that the observed variation with $\theta$ is more like $\cos ^{\frac{2}{3}} \theta$, although there is no theoretical justification for expecting the variation should be a power of $\cos \theta$.

The second method consisted of measuring the lunar heat from the subsolar point as it sweeps across the surface through a lunation. Figure 3 shows the geometry relevant to this measurement. The subsolar point is visible from first-quarter to third-quarter Moon; the phase angle is designated by $\alpha$. The results of these measurements by Pettit and Nicholson (1930) and Sinton (1962) are shown in Fig. 4, where the dashed curve represents the constant value expected for a Lambert surface. The measured values can be seen to be higher than the Lambert curve for $|\alpha|<\sim 60^{\circ}$, and lower for $|\alpha|>\sim 60^{\circ}$.

Both the above types of observation can be understood qualitatively in terms of surface roughness. In the case of the Full Moon observations, the observer will see no shadows. (1) Near the limbs the observer will see surfaces (mountains, crater walls, rills) which are oriented toward him. These same surfaces will also be favorably oriented toward the Sun and will be at a higher temperature than the average level surface. The observer will see a larger percentage of illuminated, elevated surfaces at a higher temperature than the level surface; thus, the measured temperatures will be higher than the Lambert temperature. (2) Near the center of the disk at Full Moon, the observer looking normal to the surface will see the bottoms of "valleys" which will be warmer than the level surface because of reradiation. Thus, the measured temperature of the subsolar point will be higher than the Lambert temperature. 
In the case of subsolar point observations at various phases, again surface geometry is the key. When the subsolar point is observed at low elevation angles (e.g. , a few days before third quarter Moon) less of the warmer valleys and more of the cooler slopes (mountains, crater walls, rills) will be seen. The result is then a measured temperature lower than the Lambert temperature.

\section{THE INFRARED SCAN DATA OF THE BOEING SCIENTIFIC RESEARCH LABORATORIES}

The data studied in this report were obtained from infrared scans made by J. M. Saari and R. W. Shorthill of the Boeing Scientific Research Laboratories. Since the instrumental details have been described in various publications [Shorthill and Saari (1955); Saari and Shorthill (1966)], only a brief description will be presented here.

The scanning of the lunar image was accomplished using a focalplane scanner which mechanically moved the detectors in a rectangular raster. Measurements were made simultaneously of the reflected light

at $4450 \AA$ and of the infrared radiation in the 10-12 micron region to a resolution of 8 " arc. The fast response of the mercury-doped germanium infrared detector used allowed rapid scanning so that the lunar disk could be covered in 30 minutes. The measurements were made using the 60" telescope at Mt. Wilson except for the scan at $-2^{\circ}$ phase angle for which the 74" Kottamia telescope of the Helwan Observatory in Egypt was used (resolution of $10^{\prime \prime}$ of arc).

The analog signals were recorded on magnetic tape and digitized into a format suitable for reduction on an IBM-7094 computer. Certain selected signal levels were assigned coded symbols so that the output of the computer consisted of a printout of these symbols positioned as they were encountered in the scan raster. These levels were either contoured by hand or machine resulting in thermal contour maps (and associated 
photometric maps) which were used in the present work. Figure 5 is an example of such a the rmal contour map for a phase angle of $-2^{\circ} 16^{\prime}$; a librated standard orthographic grid has been overlaid to provide position information.

The calibration of the infrared detector [Saari and Shorthill (1966)] was done with respect to a calculated value of the temperature of the subsolar point, which included corrections for the Sun-Moon distance, the local albedo, and the directional effects shown in Fig. 4. Planck's blackbody function was integrated over the response of the filter-detector combination and the atmospheric window. When the subsolar point was not visible, the calibration was determined by the detector calibration made each night and optical losses in the system determined from the observations made when the subsolar point was visible.

\section{EXAMPLES OF DATA ALONG THE THERMAL MERIDIAN}

Preliminary studies in the initial phases of this work were concerned with the variation of lunar temperature from the subsolar point (SSP) along a line through the apparent center of the disk, the thermal meridian [Montgomery (1966)]. Five phases were studied $\left(-2^{\circ}, \pm 39^{\circ}\right.$, $\pm 90^{\circ}$ ). The data at Full Moon showed a reasonable agreement with the work of Pettit and Nicholson (1930). At the larger phase angles the Lambert surface law $\mathrm{T}=\mathrm{T}_{0} \cos ^{\frac{1}{4}} \theta$ seemed to be obeyed.

We show here data at four phase angles $\left(\alpha=-2^{\circ},-15^{\circ},-29^{\circ}\right.$, and $-65^{\circ}$ ) which reveal the transition from the $\cos ^{\frac{1}{6}} \theta$ law near Full Moon to $\cos ^{\frac{1}{4}} \theta$ at larger phase angles. Figure 6 shows the paths along which measurements of brightness temperature were analyzed. The phase angle $\alpha$ is negative before Full Moon.

The variation in brightness temperature along these paths is plotted in Fig. 7. The intersection of the curves with the ordinate gives the subsolar point temperature. For comparison, the $\cos ^{\frac{1}{6}} \theta$ and $\cos ^{\frac{1}{4}} \theta$ 
variations are also shown on the graph. Notice that the data for $-2^{\circ}$ phase angle more or less follow the $\cos ^{\frac{1}{6}} \theta$ law; the data actually show a slower variation toward the limbs than indicated by this law. As the phase angle increases, the data more closely approximate the $\cos ^{\frac{1}{4}} \theta$ law, although even for the larger phase angles there is a slight but noticeable upturning at large angular distances from the subsolar point.

\section{DEFINITION OF THERMAL COORDINATES}

The thermal coordinate system used in this analysis was chosen to show the variation in brightness temperature of points on the lunar surface with respect to their angular distance from the subsolar point. The data are exhibited by considering the subsolar point as the thermal north pole and the antisolar point as the south pole and then constructing circles of the rmal longitude and latitude. The thermal prime meridian is defined as the line of thermal longitude passing through the apparent disk center at the time the data were taken. Positive thermal longitude is determined according to the right-hand rule, i.e., if the thumb of the right hand points along the thermal axis and out of the subsolar point, the fingers curl in the direction of positive thermal longitude. Thus positive thermal longitude is measured counterclockwise looking down upon the subsolar point. In this system the terminator is the thermal equator, i. e., zero-degree thermal latitude.

\section{THERMAL GRID OVERLAYS AND BRIGHTNESS TEMPERATURE VERSUS THERMAL LONGITUDE - PHASE CONSTANT}

Thermal coordinate grids for every $10^{\circ}$ interval of latitude and longitude were drawn for each particular phase analyzed at the topocentric libration for the time of the measurement. These grids were projected on the thermal contour maps, and the brightness temperatures read off 
at the above grid intersections of latitude and longitude. Scan programs for nineteen separate lunar phases were analyzed, and are listed in Table I, together with the positions of the subsolar point and apparent disk center.

For reference purposes, the thermal grids for each phase (which can be thought of as being attached to the lunar surface) have been redrawn as they would appear at mean libration $(0,0)$ and laid over the ACIC LEM lunar chart. Figure 8 shows this lunar chart without the thermal grid lines; the sensor resolution is indicated by a small circle 1/240 of the lunar diameter.

The thermal grids for each phase together with the corresponding plots of brightness temperature versus thermal longitude at constant phase angle for each circle of thermal latitude are shown in Figs. 9 through 46. Progressing across a given curve corresponds to traveling around a circle of the rmal latitude concentric with the subsolar point. The circled values indicate measurements taken in upland (rough) regions; the values with vertical bars in smooth regions such as maria. The arrow on each grid system indicates the direction of positive thermal longitude and the symbol $\uparrow$ indicates the apparent disk center.

\section{BRIGHTNESS TEMPERATURE VERSUS THERMAL LONGITUDE - LATITUDE CONSTANT}

A second method for presenting the data is shown in the next 15 figures. In this system the results for a particular thermal latitude are plotted against the thermal longitude for the different phases. The data for the negative phases are shown in Figs. 47 through 54, and for the positive phases in Figs. 55 through 62 . 


\section{BRIGHTNESS TEMPERATURE VERSUS PHASE - LATITUDE CONSTANT}

The final method of data presentation is shown in Figs. 63 through 70. Here the brightness temperatures for particular thermal latitudes are plotted as a function of the phase angle $\alpha$, with points shown for $30^{\circ}$ increments of thermal longitude. The data are presented with reference to the thermal coordinate system described above. Because of the convention chosen, a peculiarity arises when a given the rmal coordinate is observed through a lunation. Consider the point at thermal latitude $40^{\circ}$ and thermal longitude $30^{\circ}$ when $\alpha<0^{\circ}$, i.e., before Full Moon. In Fig. 13, where $\alpha=-91^{\circ} 52^{\prime}$, this point falls south of the crater Santbech. The point moves west to an area on the southeast rim of Mare Nectaris at a phase angle $\alpha=-88^{\circ} 41^{\prime}$ as seen in Fig. 15. In Fig. 17, at $\alpha=-65^{\circ} 29^{\prime}$, the point has moved to a region northwest of Sacrobasco and just before Full Moon; at $\alpha=-2^{\circ} 16^{\prime}$, the point is on the western rim of Mare Humorum (see Fig. 25). The brightness temperature up to this point has been increasing toward Full Moon and is plotted on Fig. 67 with a $\square$ symbol.

At a phase angle $\alpha=+11^{\circ} 42^{\prime}$, however, in this grid system the point of thermal latitude $40^{\circ}$ and thermal longitude $30^{\circ}$ has shifted to a region near Maraldi (see Fig. 27). It is clear that the point on the thermal grid we are actually interested in following now has a thermal longitude of $-150^{\circ}$ and is plotted on Fig. 67 with a $\Delta$ symbol which places it near the crater Mersenius. This effect is shown in Fig. 71 where the brightness temperature versus phase angle has been plotted for a family of thermal latitudes for $30^{\circ}$ thermal longitude before Full Moon $\left(\alpha=0^{\circ}\right)$ and for $-150^{\circ}$ thermal longitude after Full Moon. It is interesting to note that under these conditions the brightness temperature continues to increase, reaching its maximum somewhat after Full Moon. This effect, a consequence of the directional properties of the lunar thermal emission, will be discussed later. In general then, in order to follow a certain grid point, which moves across the lunar disk from east to west along a band 
of selenographic latitude, as the phase angle increases, one subtracts $180^{\circ}$ from the thermal longitude at minus phase angles to obtain the proper the rmal longitude to follow for positive phase angles.

\section{FACTORS AFFECTING THE BRIGHTNESS TEMPERATURE VALUES}

This report is concerned with the directional characteristics of lunar infrared emission. There are two factors which affect the measured brightness temperatures and contribute to the scatter in the data, namely variations of local albedo and surface topography.

Consider first the effect of albedo. On the Moon the albedo ranges from 0.05 to 0.20 ; typical maria have a value of 0.06 , and typical uplands a value of 0.12 . A determination was made on the scan program for phase $-2^{\circ} 16^{\prime}$ of the change in brightness temperature in traversing from mare to upland regions. It was found to amount to approximately 2 percent; thus at $400^{\circ} \mathrm{K}$, for example, an $8^{\circ} \mathrm{K}$ difference in temperature can be expected between mare and uplands.

With regard to the effect of topography, consider the change in temperature caused by $a 5^{\circ}$ change in the angle between the Sun and surface normal. Using the $\cos ^{\frac{1}{4}} \theta$ law, one calculates for $\theta=30^{\circ} \pm 5^{\circ}$ that the temperature will be $376^{\circ}+4^{\circ}$; likewise for $\theta=60^{\circ} \pm 5^{\circ}$, the temperature will be $318^{\circ}+11^{\circ}$. Topography will have its greatest effect near the terminator, and least effect near the subsolar point.

The above examples serve to point out the variation in the measured brightness temperature to be expected from these effects. Examination of the temperature plots shows that the scatter of the points is, in fact, in this range. 


\section{DISCUSSION}

In the first set of figures (Figs. 10, 12, . 44, and 46), since each curve on each graph is at constant latitude (i.e., a fixed Sun elevation angle to the surface normal), the brightness temperature should be constant for a Lambert surface. The main feature evident on these plots is the concave upward shape of the curves, which is particularly noticeable for the smaller values of thermal latitude and for $|\alpha|$ between $11^{\circ}$ and $76^{\circ}$. Perhaps the best example of this effect is shown in Fig. $24\left(\alpha=-15^{\circ} 28^{\prime}\right)$ for thermal latitude of $10^{\circ}$.

The figures in the second set (Figs. 47 through 62) are each plotted for a particular thermal latitude as a function of phase angle and thermal longitude. Since the the rmal latitude is constant for each figure, the Sun angle to the surface is constant and a Lambert surface would have a constant brightness temperature. On each figure a Lambert temperature has been indicated, which is 3 percent less (to correct for an albedo midway between mare and uplands) than the theoretical blackbody temperature. For $|\alpha|$ less than about $50^{\circ}$, the measured brightness temperature is greater than the Lambert temperature. This can be understood as implying that surfaces oriented toward the Sun, and hence receiving more energy per unit area than level surfaces, are contributing more to the integrated brightness temperature than are surfaces oriented away from the Sun.

The concave upward shape of the curves in the first set of figures (Figs. 10, 12, ., 44, and 46) and in the second set (Figs. 47-62) at small the rmal latitudes appears to be due to the changing ratio of illuminated area to shadowed area seen by the sensor. The curves for high thermal latitudes are quite flat. This is to be expected since closer to the subsolar point there are fewer shadows. Figures 63 through 70 emphasize this effect. As the phase angle increases, the ratio of illuminated to shadow region within the scan zone decreases, and the integrated brightness 
temperature declines. In Fig. 71 a crater is shown near the terminator on the thermal meridian; most of the crater interior is in shadow. If the crate $r$ is moved in the rmal longitude (at the same thermal latitude), the percentage of the crater observed to be in shadow will drop; therefore, the brightness temperature will increase.

Thus, two effects, both caused by surface geometry, explain the data. At small phase angles there are few shadows and surface orientation toward or away from the Sun is the dominant effect. At larger phase angles, there are more shadows within the scan zone and the integration over shadowed and illuminated surfaces is the dominant effect.

A closer examination of the data reveals that the maximum brightness temperature is not necessarily observed exactly in the direction of the Sun (see Fig. 72). This is in contrast to the photometric scattering function, for which all points on the Moon, regardless of position, show a sharp maximum in brightness at Full Moon. The result for the infrared can be understood qualitatively in terms of a simplified model consisting of a flat surface interspersed with many craters in the field of view. The craters are assumed to be portions of a hemisphere with no rims (see Fig. 73). Consider the Sun illuminating the surface at an elevation angle as shown. At point $C$, where the Sun's rays are perpendicular to the crater interior, the temperature will have a maximum value designated as $T_{3}$. Just inside the crater rim at $D$ the temperature will be less, at a value $T_{2}$. There will be a corresponding point at $B$ at the same temperature. The shadow edge at $A$ will be at a temperature $T_{1}$, which is lower than $T_{2}$. Let us now consider the variation in brightness temperature as the observer's elevation angle changes from that of the Sun, downward until he is looking parallel to the surface. Since the crater occupies a constant percentage of the field of view regardless of the observer's elevation angle, we can ignore the effect of the flat surface outside the crater and consider only the variation in brightness temperature from the crater itself. Now, when looking down the Sun line, the observer sees all 
of the illuminated portion of the crater from $A$ to $D$ at a certain average brightness temperature. If the observer's elevation angle is reduced so he sees only the portion from $B$ to $D$, the brightness temperature will increase, since he no longer can see $A B$, all of which is at a temperature lower than $T_{2}$. If now the observation angle is further reduced so only $C D$ is visible, the same brightness temperature will be noted as when BD was visible, a value somewhere between $T_{2}$ and $T_{3}$. When the surface is observed just at the grazing angle, only the region near $D$ is visible, so the brightness temperature will be $T_{2}$. Clearly a maximum is observed when the region of visibility extends from $D$ to somewhere between $B$ and $C$, when the elevation angle of the observer is lower than that of the Sun. Strictly speaking, the above argument applies to a cylindrical trench, but similar considerations would apply to a crater so that the same conclusion would be reached.

Qualitatively, therefore, the directional characteristics of the lunar infrared emission can be understood in terms of surface roughness, without invoking explanations such as directional emissivity. It would be possible to perform theoretical studies on various models of surface roughness to determine whether the experimentally observed data can be fitted with simple and realistic geometries. Calculations have been performed for a V-shaped trench model covering the entire surface and it was determined that, if the sides were inclined $30^{\circ}$ from the horizontal, a reasonable agreement was obtained with the brightness temperature variation observed on the Full Moon and along the thermal meridian for the quarter phases. This model, however, is not realistic, and it is suggested that a surface consisting of craters would be a better one to study. Such calculations would determine whether the observed directional effects are consistent with the geometry observed in the Ranger and Lunar Orbiter photographs. If the data could be reasonably represented by such a theoretical model, it would allow the determination of directional effects 
for wavelengths other than the 10-12 micron band employed here, and for the total energy as well, which is of interest for heat-balance calculations for spacecraft on or near the lunar surface. 


\section{REFERENCES}

Montgomery, C. G. (1966). "Empirical Determination of an Expression for Predicting the Lunar Thermal Emission at Various Angles of Observation". Interim Progress Report RPL-13870-1, Research Laboratories, Brown Engineering Company, Inc., Huntsville, Alabama.

Pettit, E., and Nicholson, S. B. (1940). "Lunar Radiation and Temperatures". Astrophys. J. 71, 102-135.

Saari, J. M., and Shorthill, R. W. (1966). "Isothermal and Isophotic Contours for the Lunar Disk through a Lunation." Boeing Document No. Dl $-82-0552-1$.

Shorthill, R. W., and Saari, J. M. (1965). "Radiometric and Photometric Mapping of the Moon through a Lunation." Ann. Y. Y. Acad. Sci. 123, Art. 2, 772-739.

Sinton, W. M. (1962). "Temperatures of the Lunar Surface." Physics and Astronomy of the Moon (Z. Kopal, ed.), Chap. 11, Academic Press, New York. 


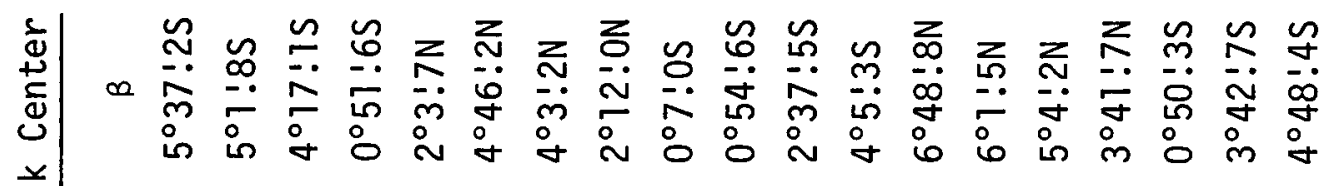

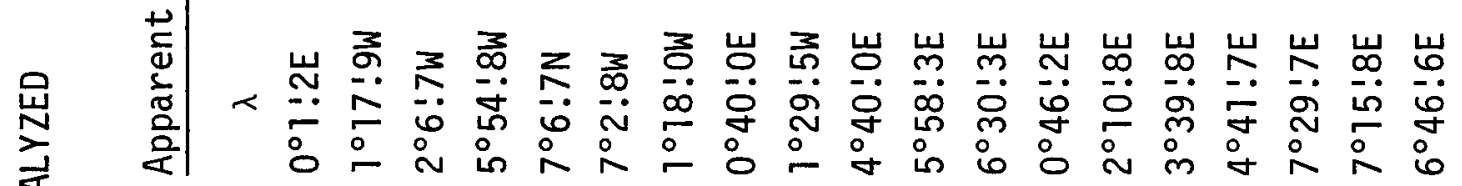

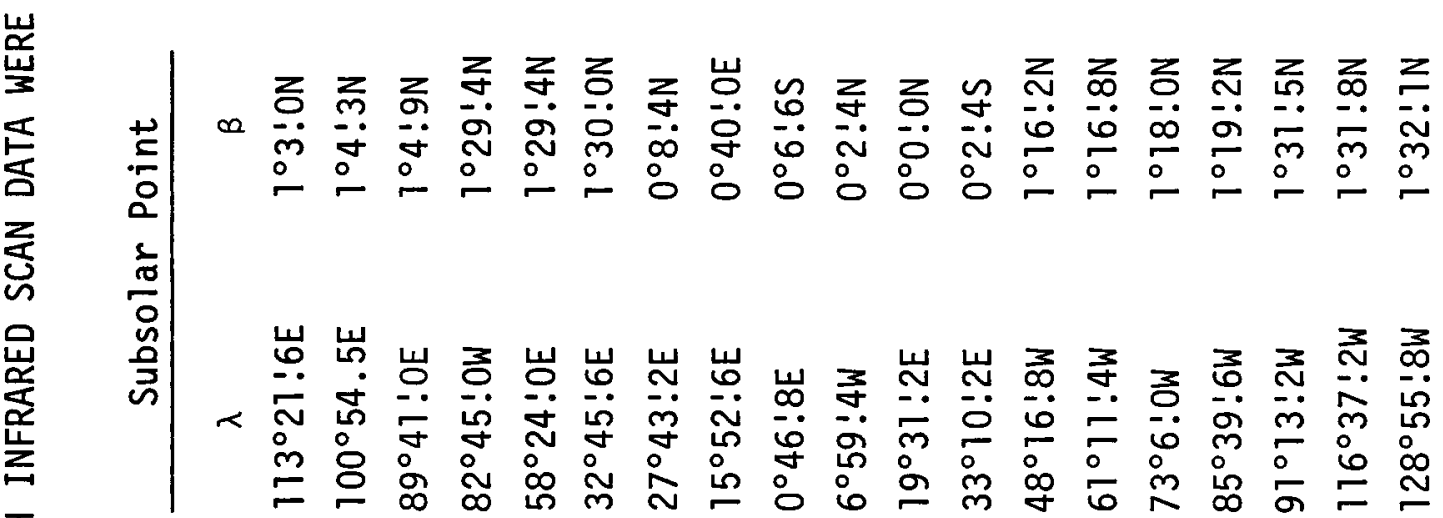

$$
\begin{aligned}
& \text { 곱 }
\end{aligned}
$$

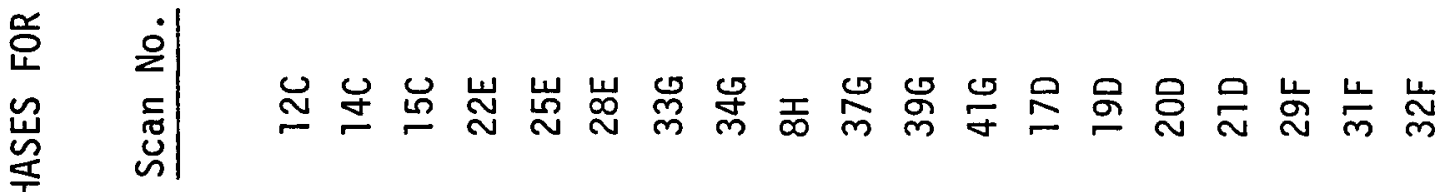

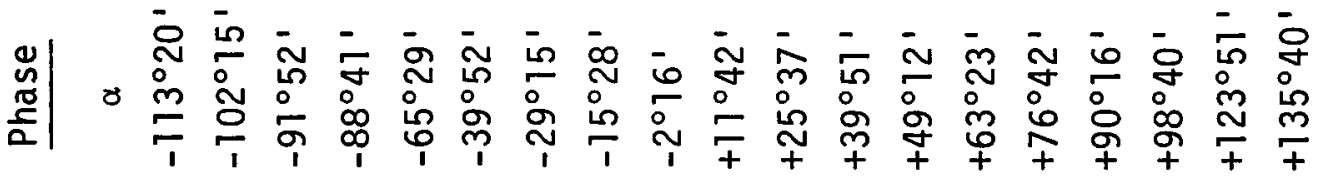

$$
\begin{aligned}
& \text { 言 }
\end{aligned}
$$




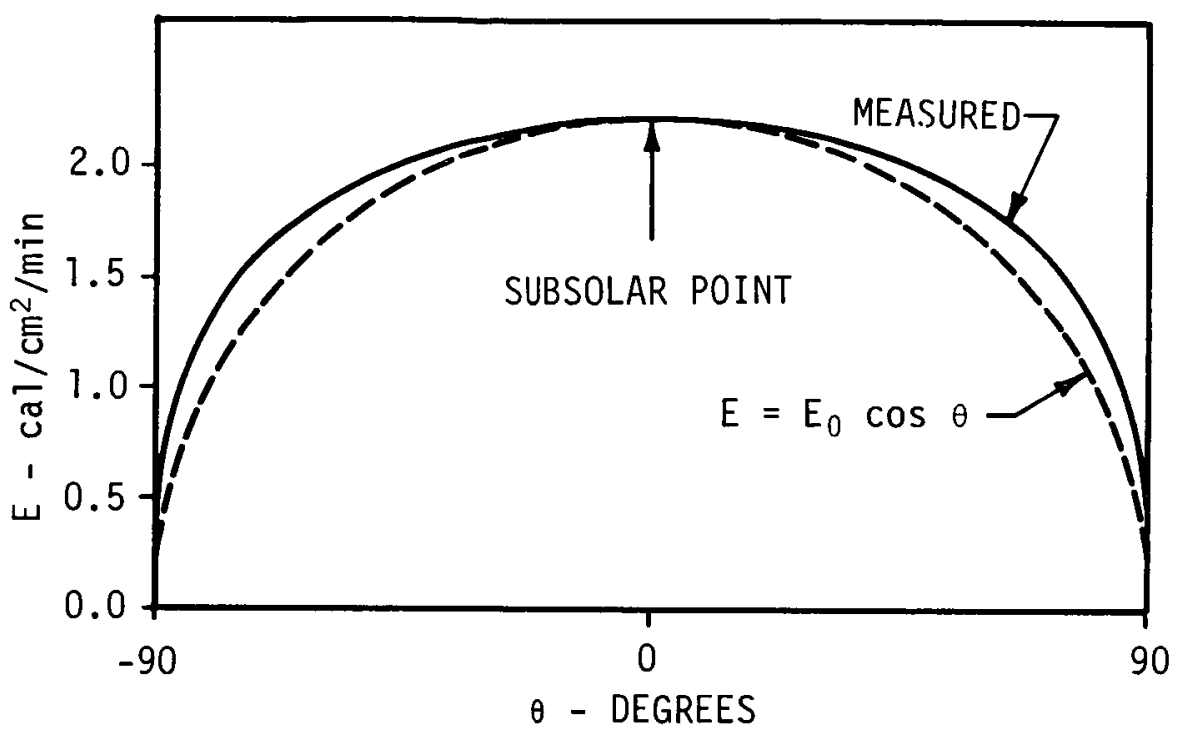

Figure 1. Distribution of Lunar Heat with Angular Distance from the Subsolar Point

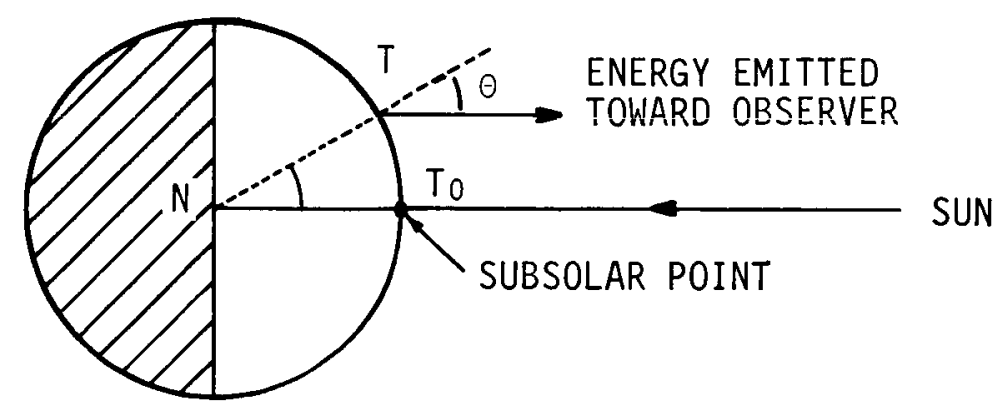

Figure 2. View Looking Down on the North Pole of the Moon. The Sun's radiation is incident from the right, and the observer is on the Sun-Moon line. 
(a) FIRST QUARTER

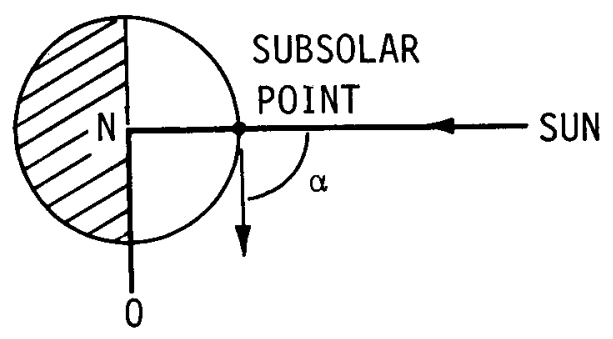

(b) WAXING GIBBOUS

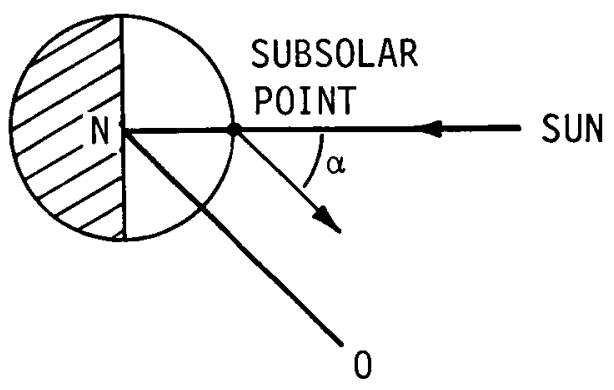

(c) FULL

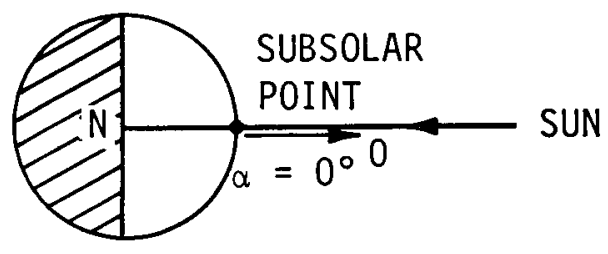

(d) WANING GIBBOUS

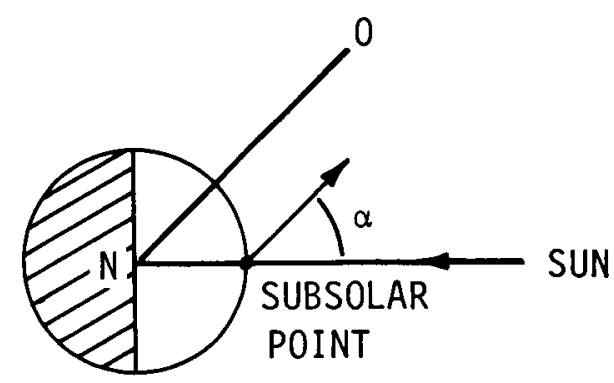

Figure 3. Progression of the Subsolar Point Around the Earthside Hemisphere of the Moon. The Sun's radiation is incident from the right; $\alpha$ is the phase angle, and the observer is at 0 . 


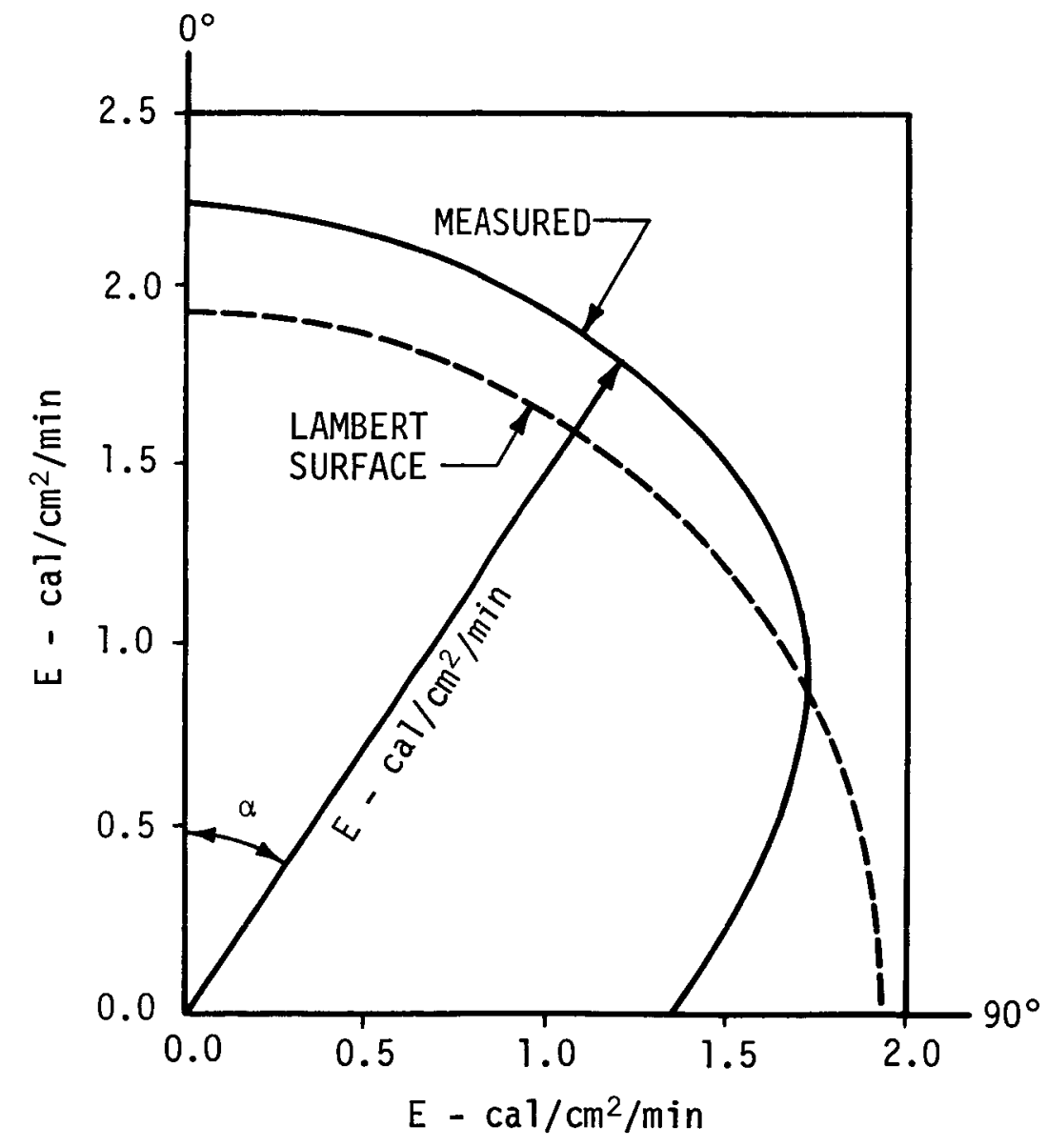

Figure 4. Polar Plot of Lunar Heat from the Subsolar Point as a Function of Phase Angle $\alpha$ 


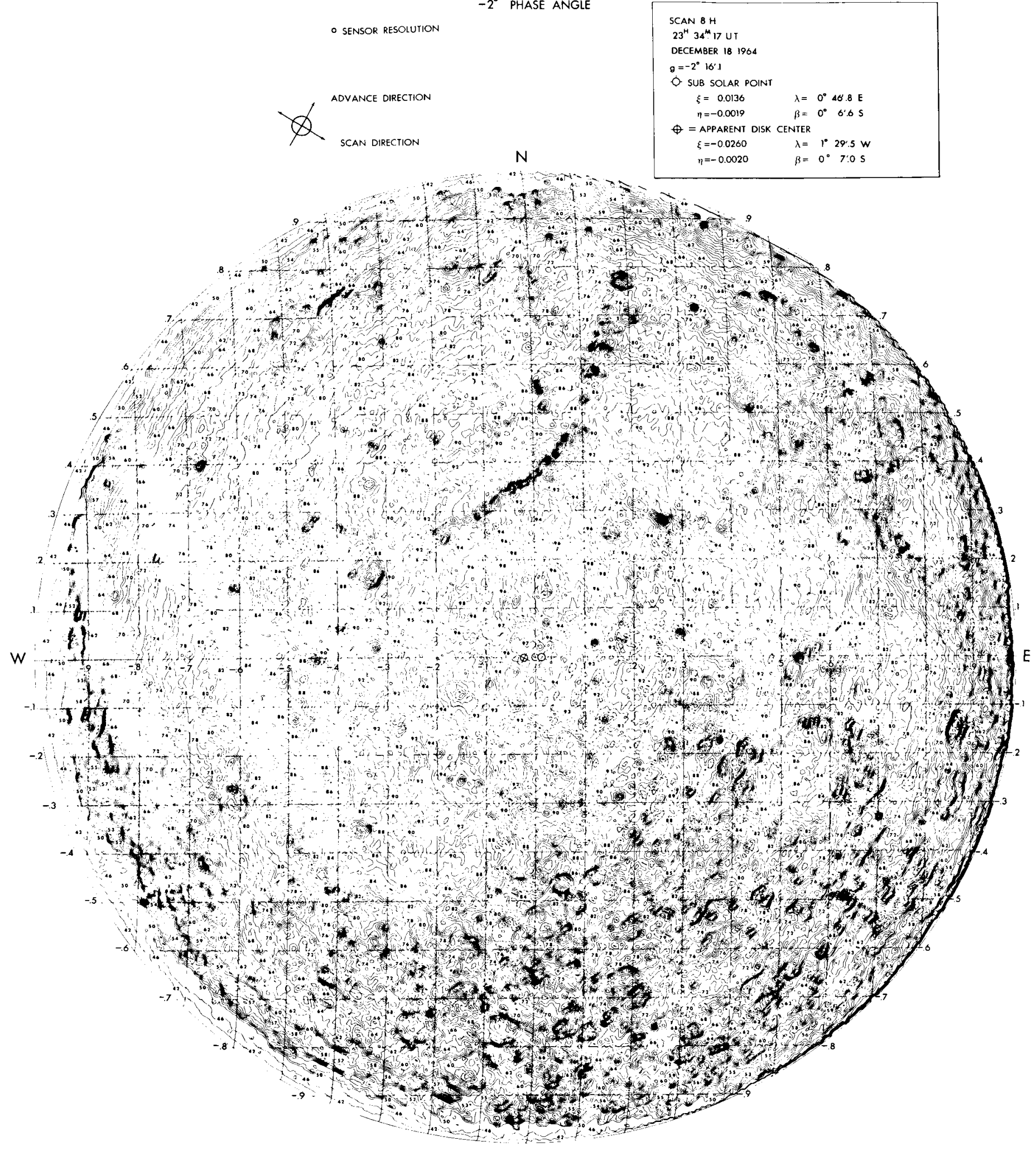

$\mathrm{S}$

BOEING SCIENTIFIC RESEARCH LABORATORIES.1968

Figure 5. Isothermal Contours for $-2^{\circ} 16^{\prime}$ Phase Angle with a Standard Orthographic Grid Overlay 

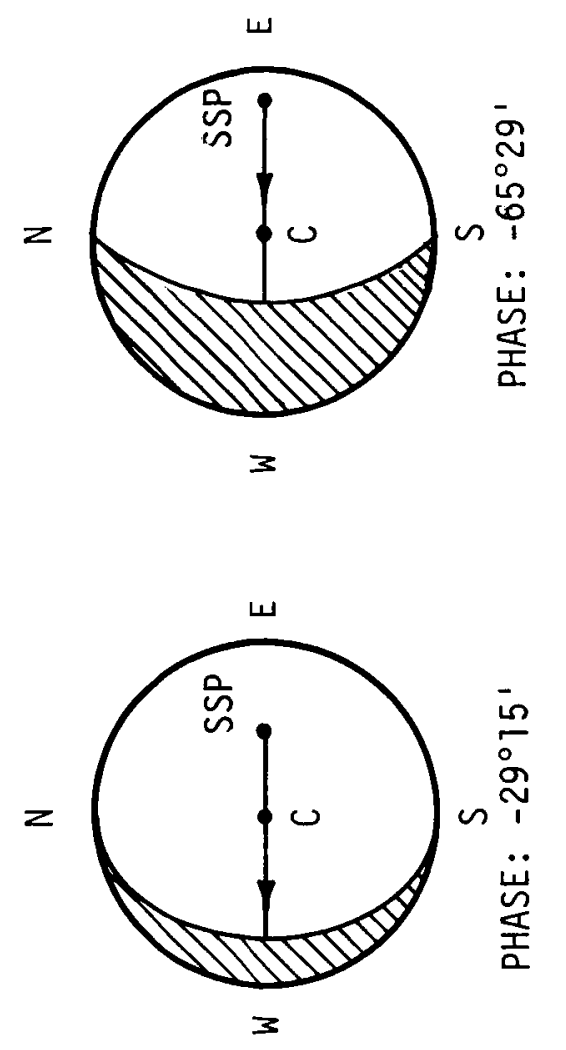

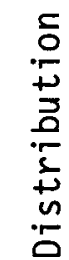

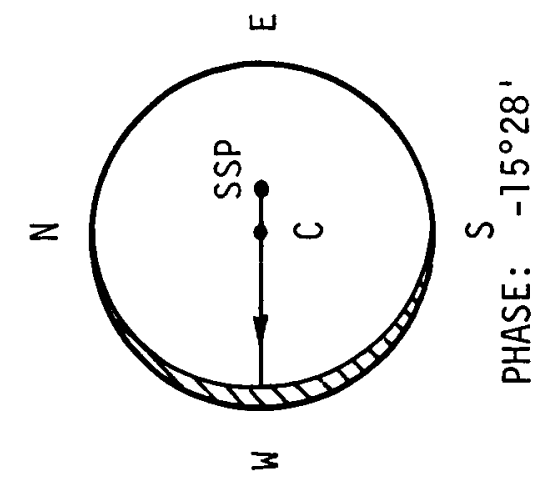

宪

$\frac{9}{+1}$

岕

월

言宁

离

ผ

돈도

돈

我

岁告

$\circ$

豙

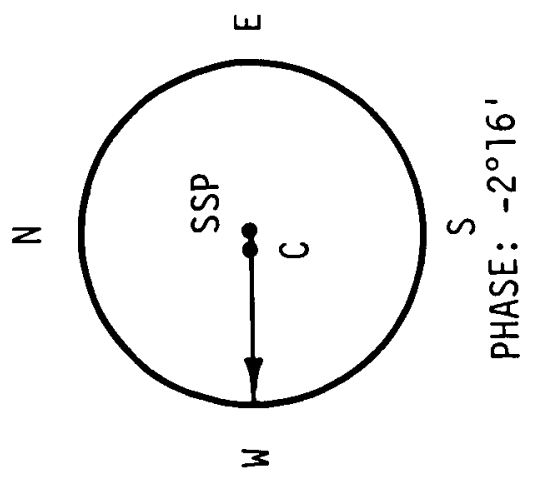




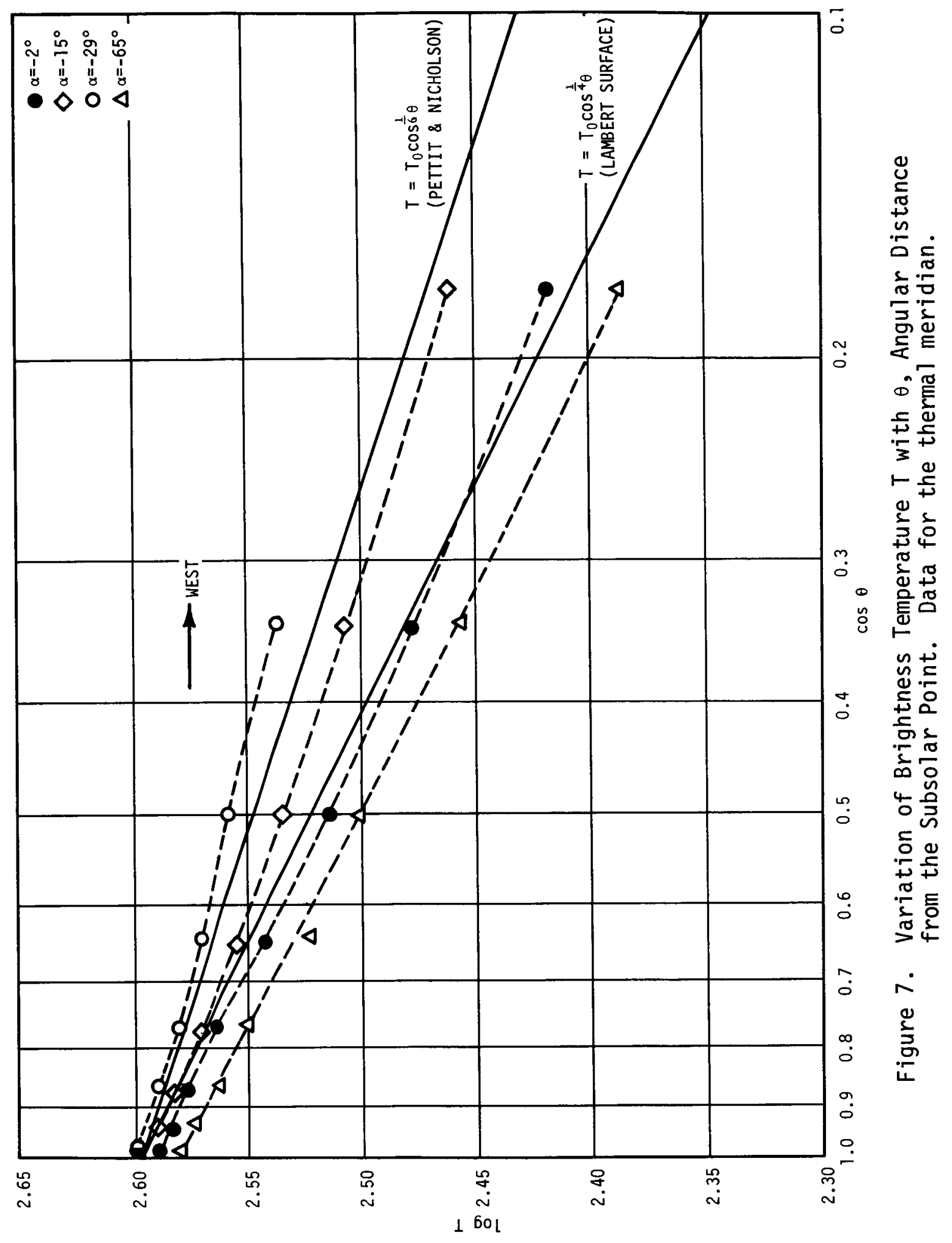






Figure 8. USAF Lunar Earthside Mosaic (LEM-1) Produced by the Aeronautical Chart and Information Center, St. Louis, Missouri 


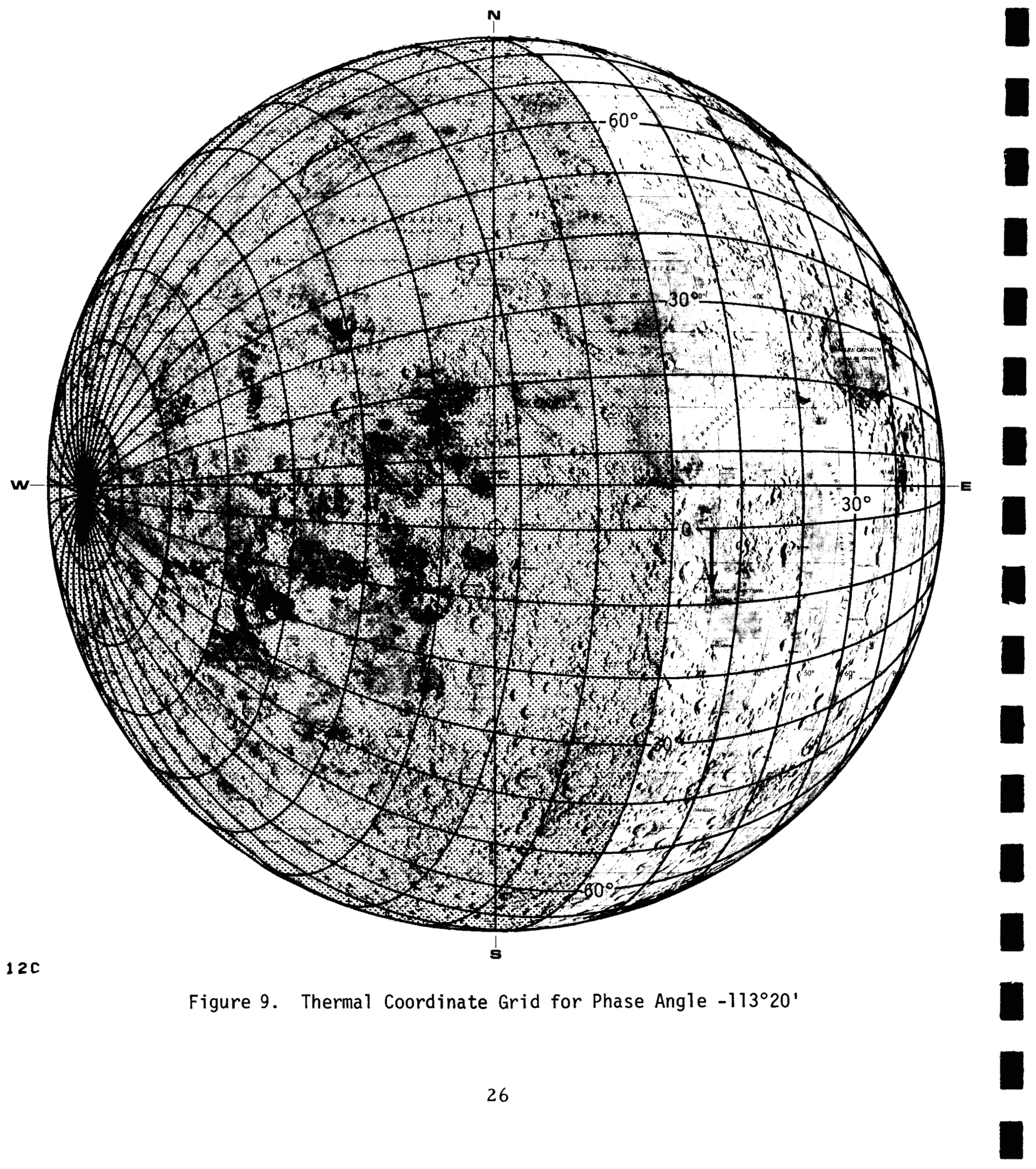




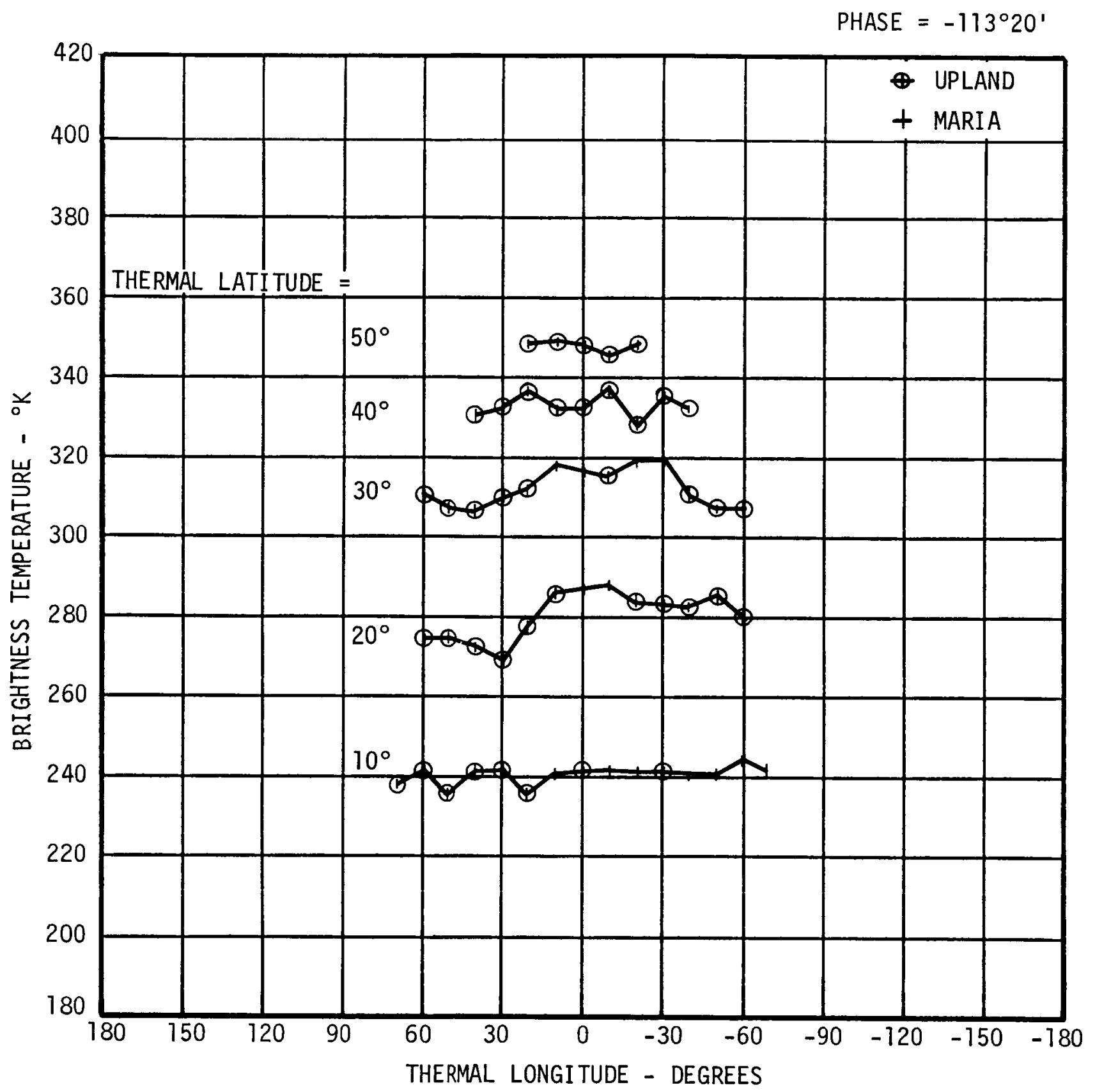

Figure 10. Brightness Temperature Versus Thermal Longitude at Phase $-113^{\circ} 20^{\prime}$ 


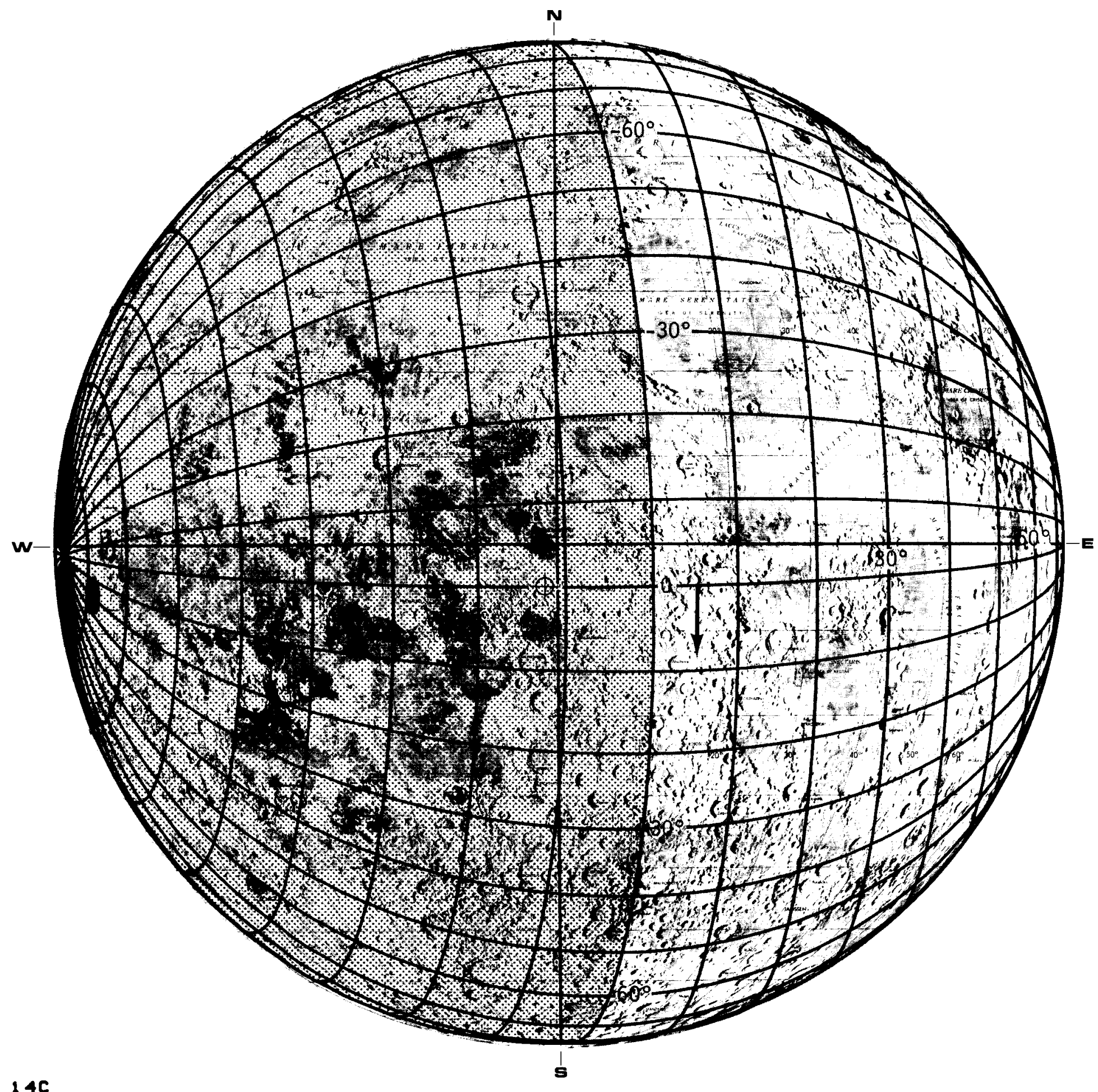

$14 \mathrm{C}$

Figure 11. Thermal Coordinate Grid for Phase Angle $-102^{\circ} 15^{\prime}$ 


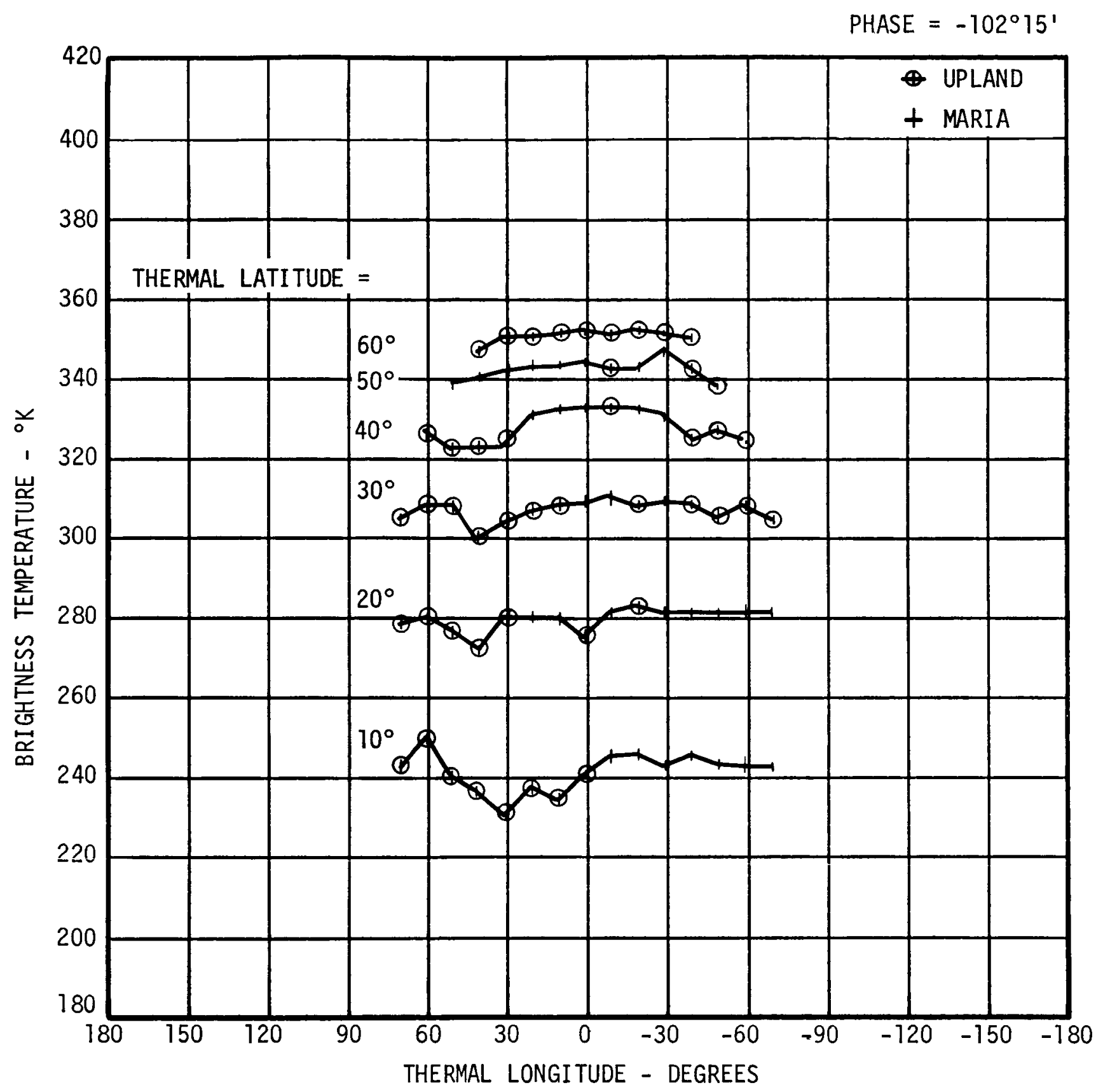

Figure 12. Brightness Temperature Versus Thermal Longitude at Phase $-102^{\circ} 15^{\prime}$ 


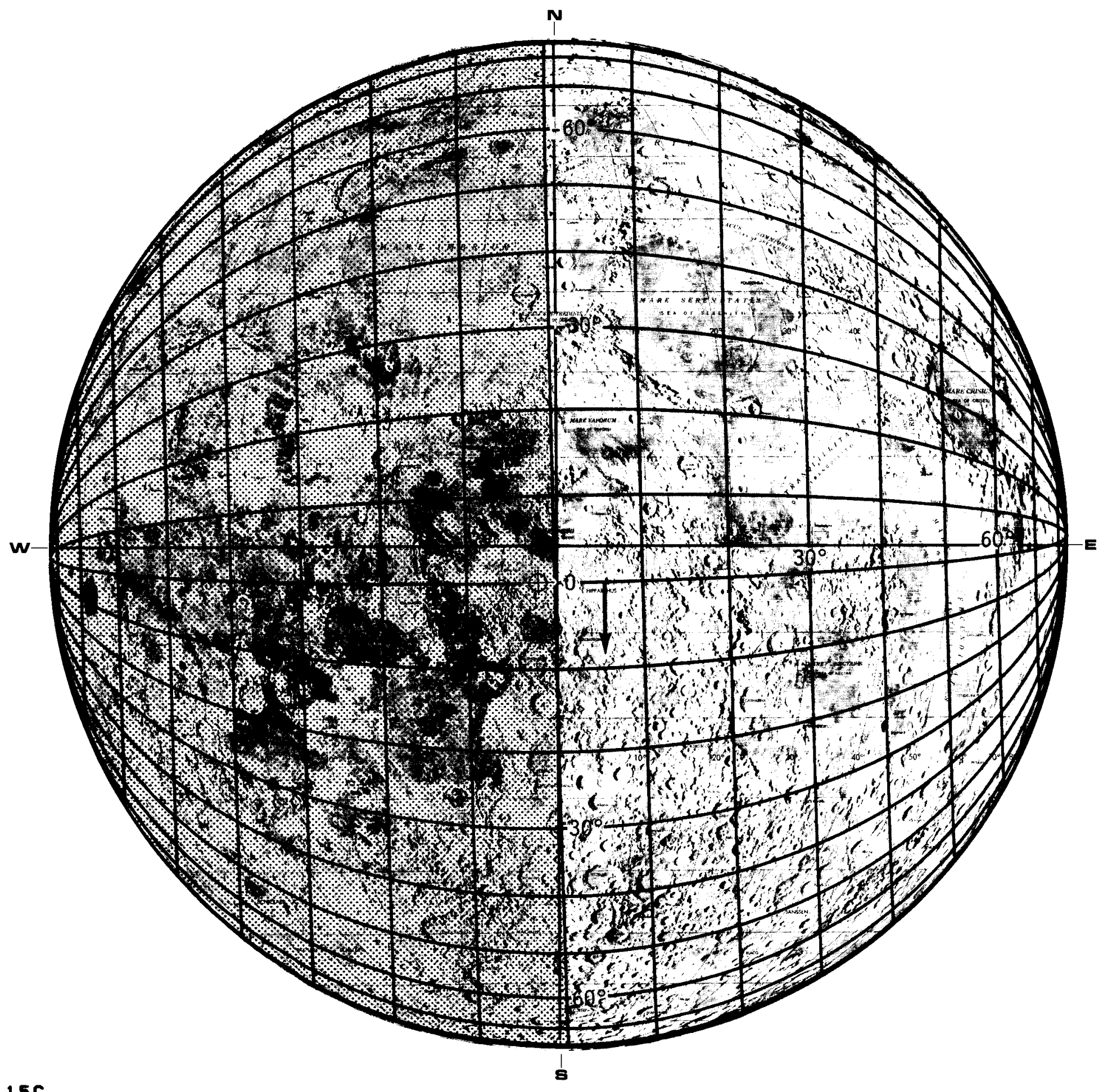

15C

Figure 13. Thermal Coordinate Grid for Phase Angle $-91^{\circ} 52^{\prime}$ 


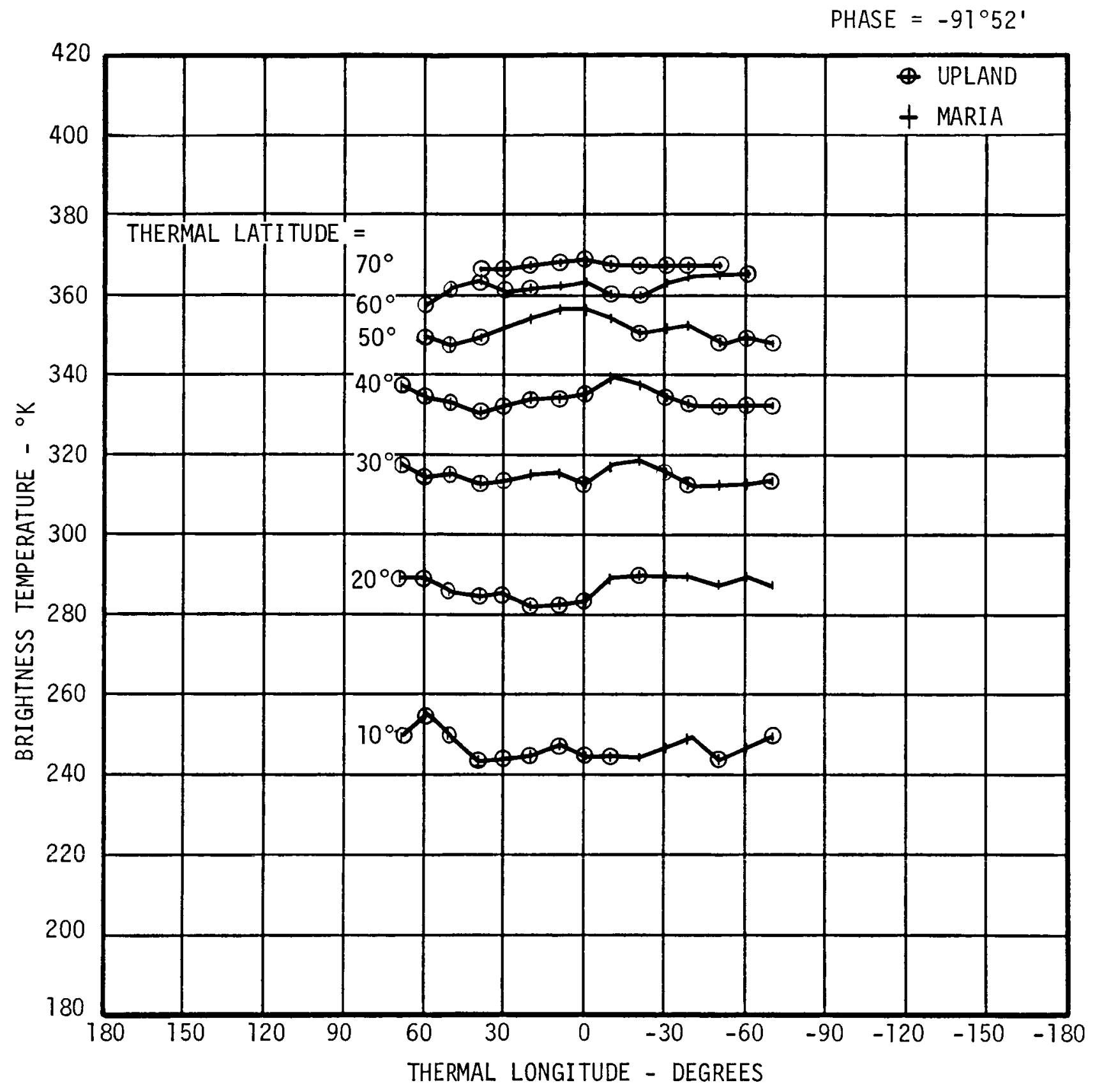

Figure 14. Brightness Temperature Versus Thermal Longitude at Phase $-91^{\circ} 52^{\prime}$ 


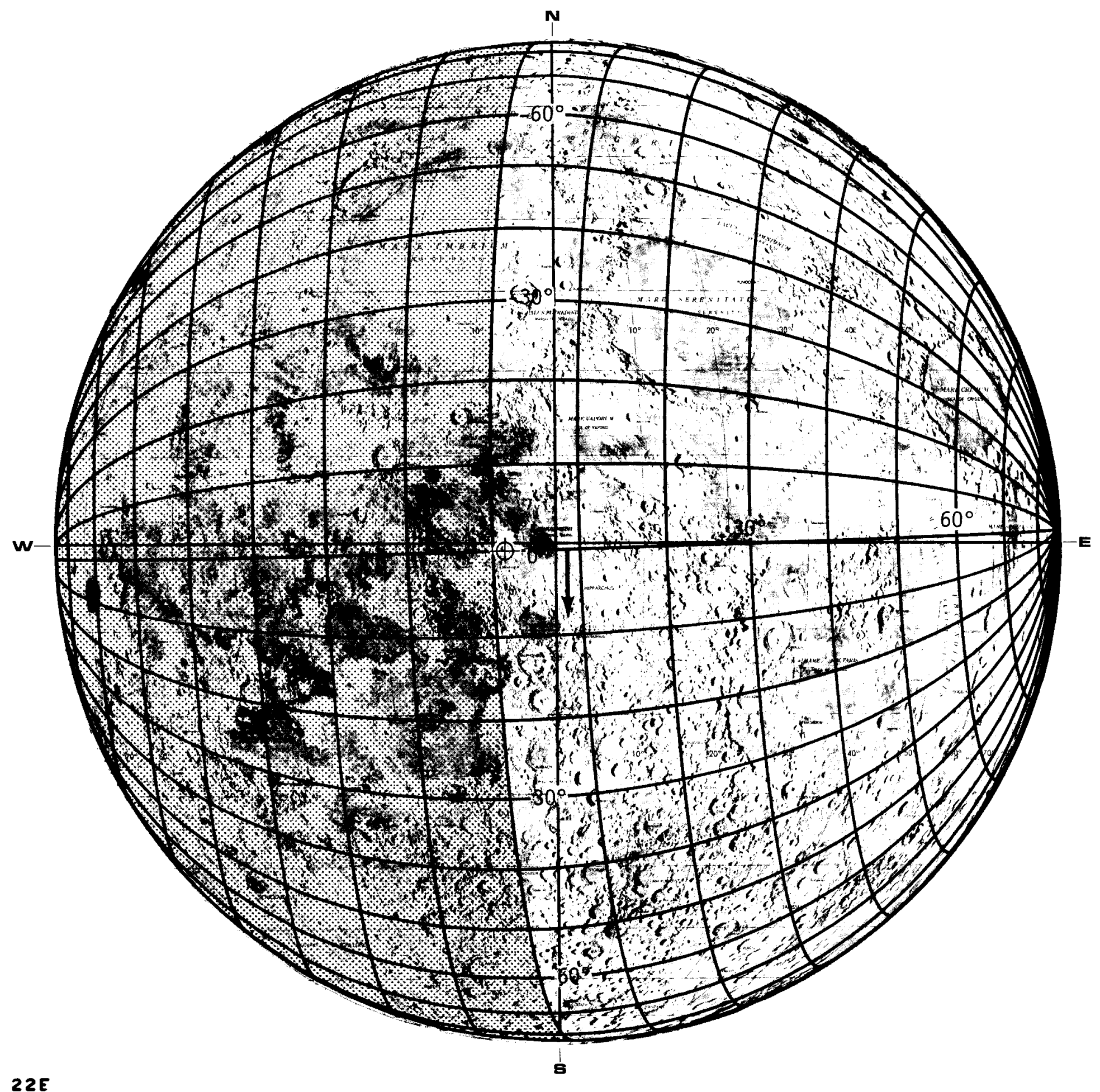

Figure 15. Thermal Coordinate Grid for Phase Angle $-88^{\circ} 41^{\prime}$ 


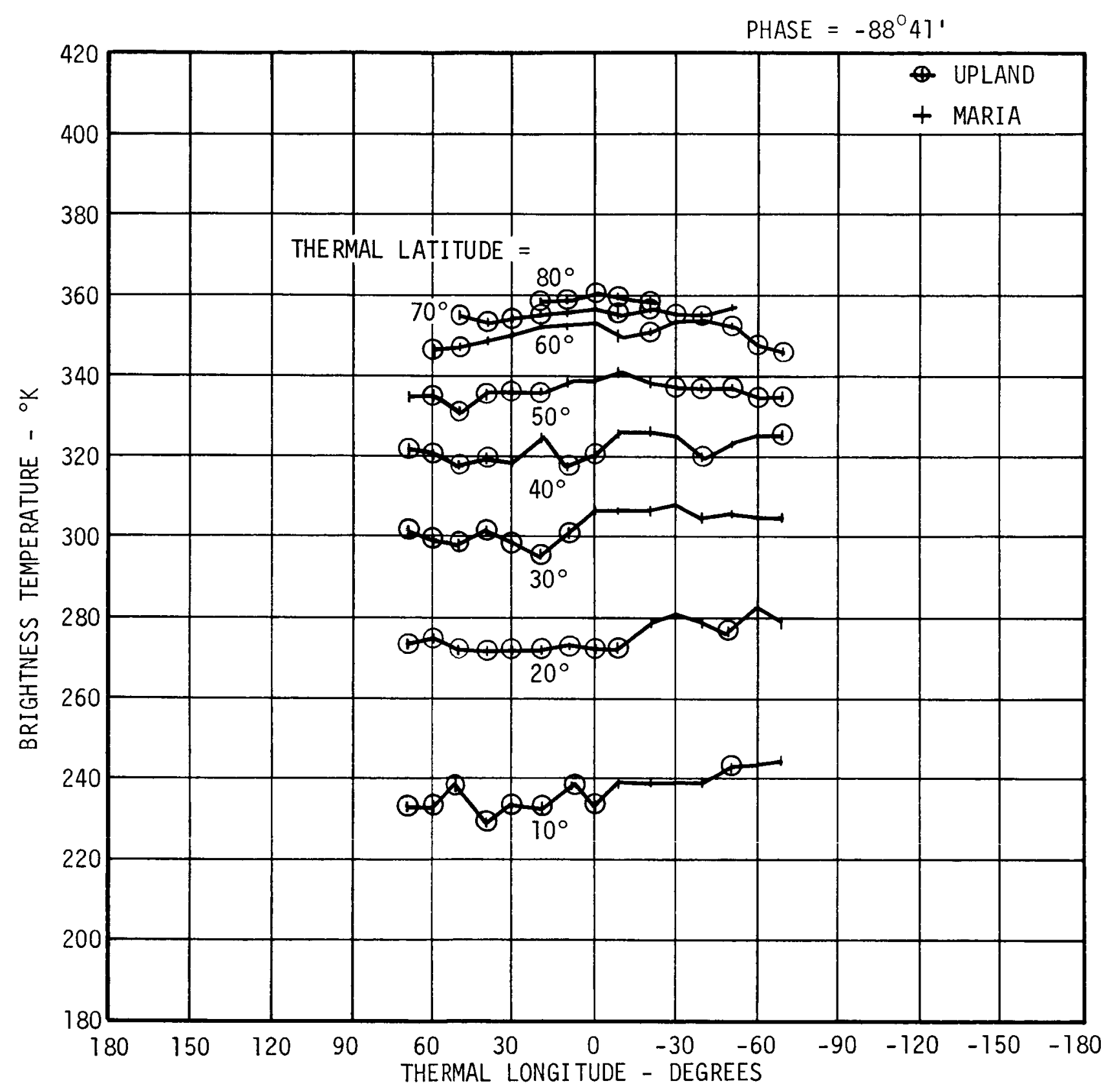

Figure 16. Brightness Temperature Versus Thermal Longitude at Phase $-88^{\circ} 41^{\prime}$ 


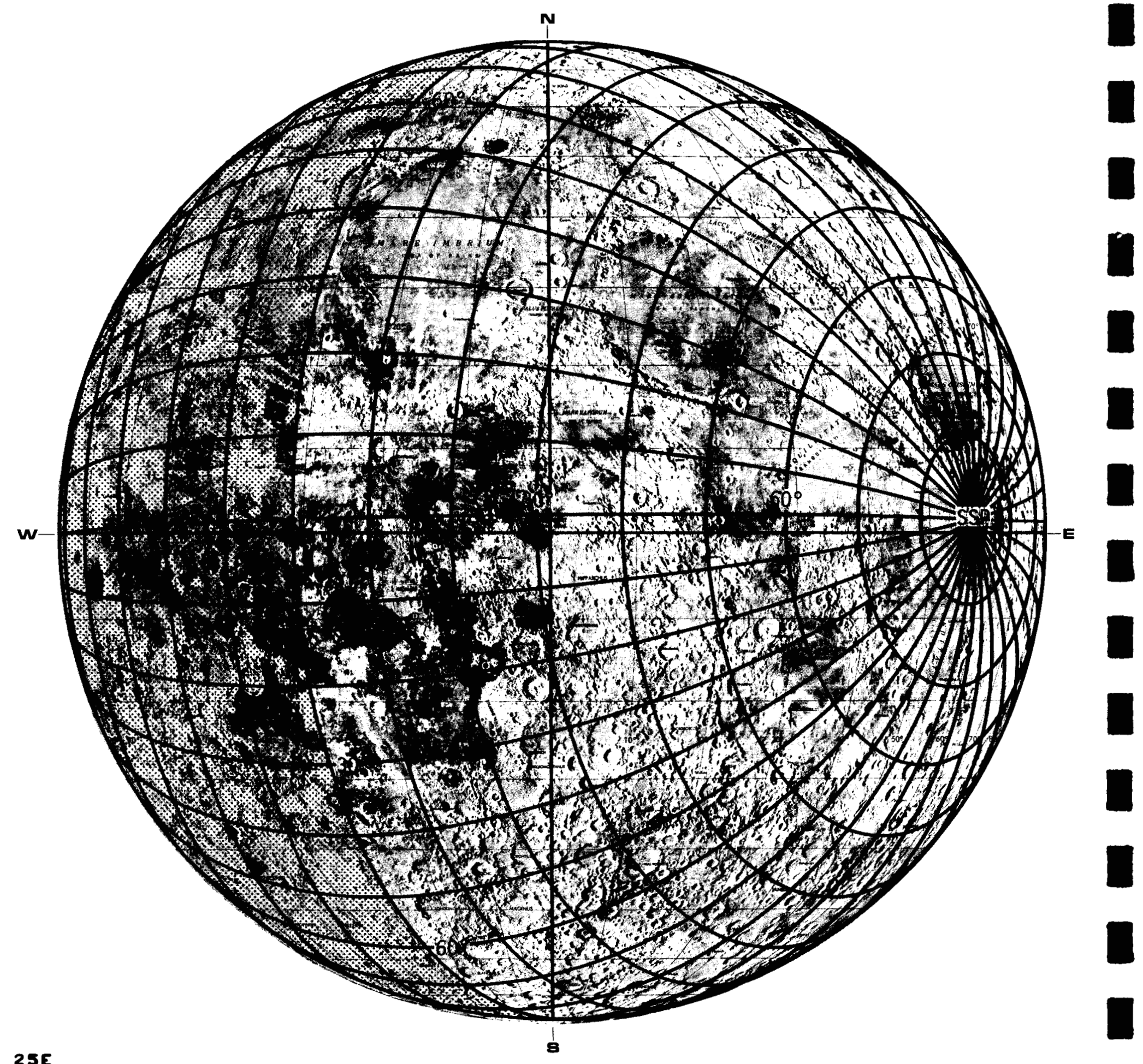

Figure 17. Thermal Coordinate Grid for Phase Angle $-65^{\circ} 29^{\prime}$ 


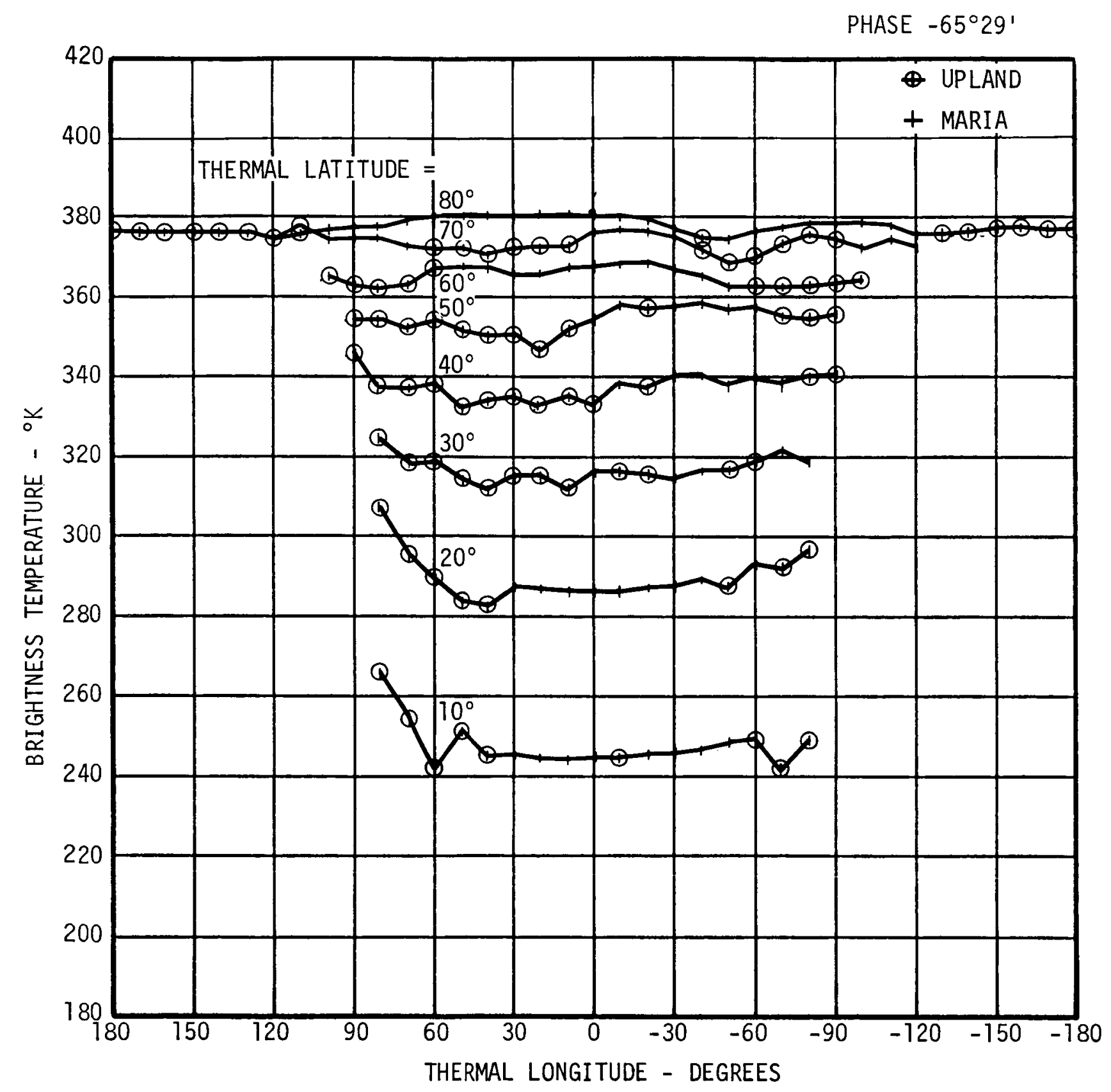

Figure 18. Brightness Temperature Versus Thermal Longitude at Phase $-65^{\circ} 29^{\prime}$ 


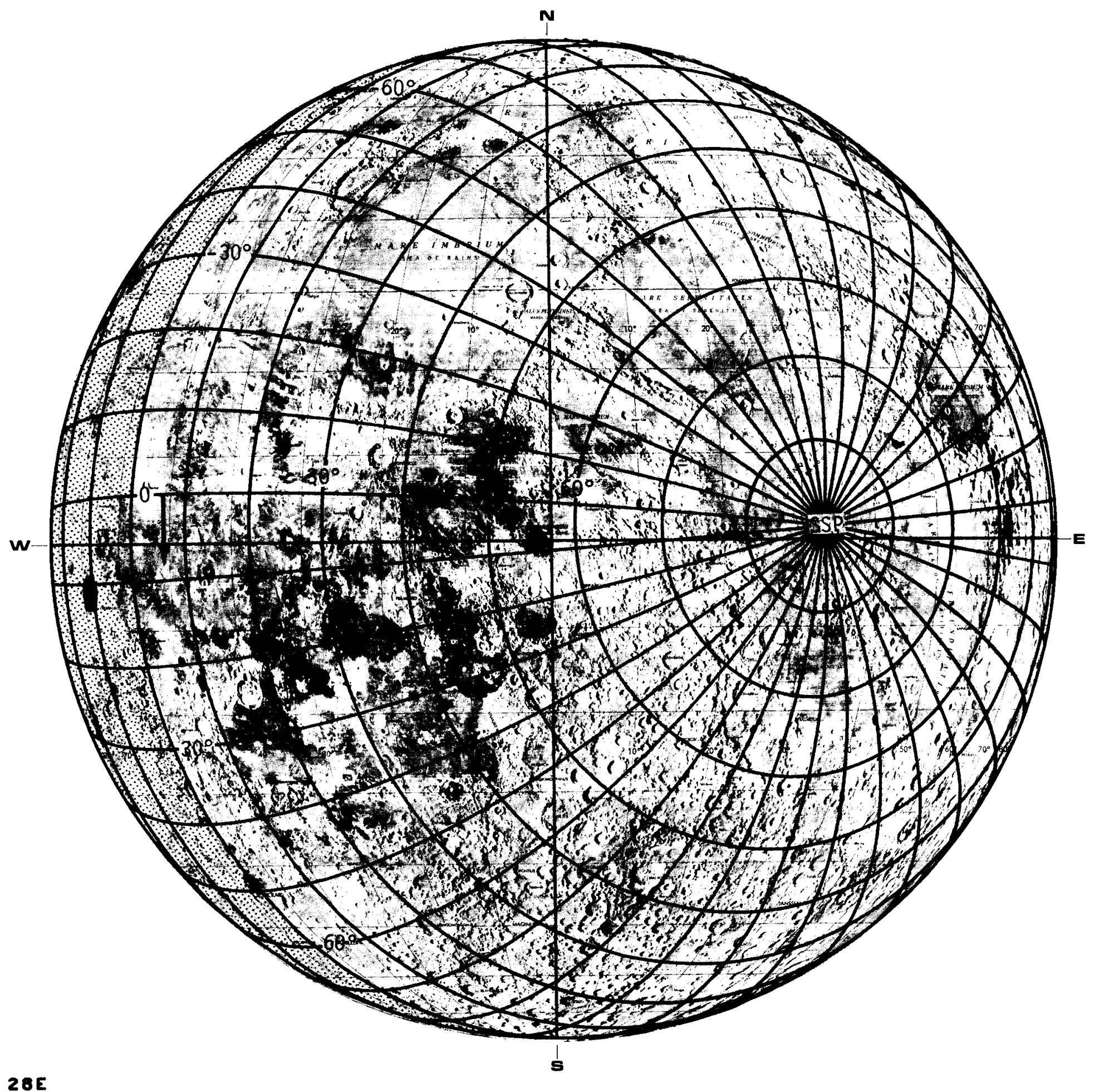

Figure 19. Thermal Coordinate Grid for Phase Angle $-39^{\circ} 52^{\prime}$ 


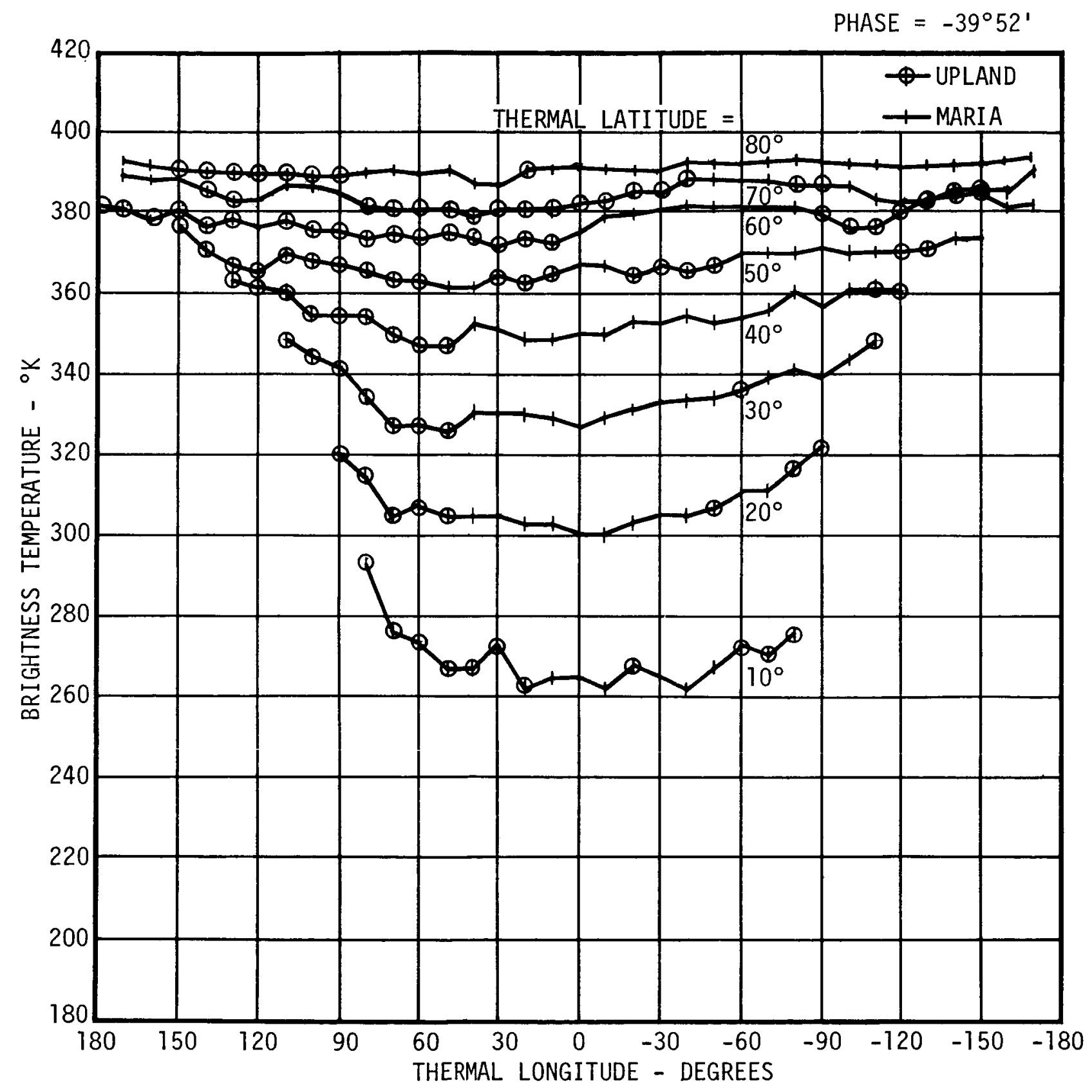

Figure 20. Brightness Temperature Versus Thermal Longitude at Phase $-39^{\circ} 52^{\prime}$ 




Figure 21. Therma1 Coordinate Grid for Phase Angle $-29^{\circ} 15^{\prime}$ 


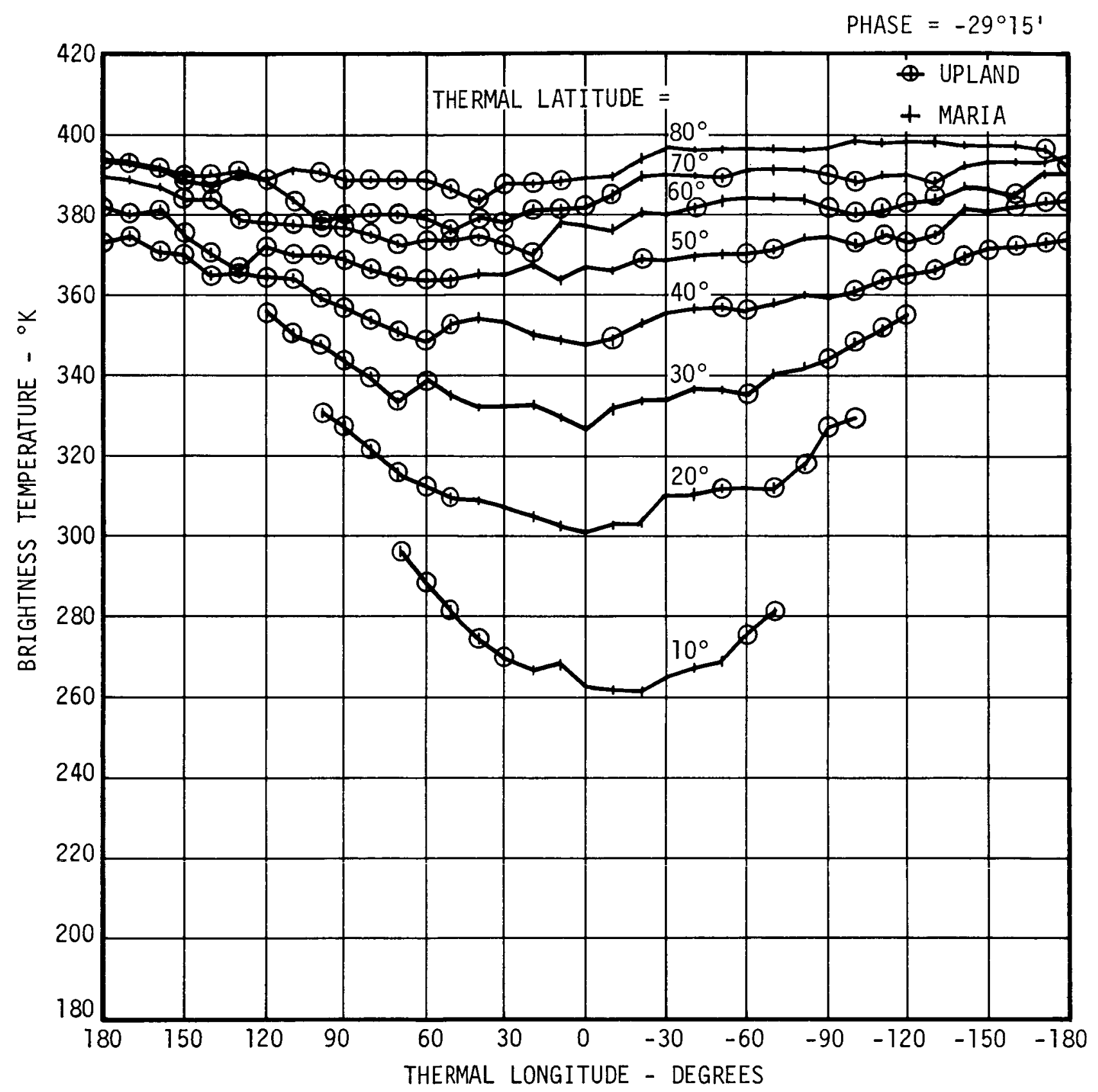

Figure 22. Brightness Temperature Versus Thermal Longitude at Phase $-29^{\circ} 15^{\prime}$ 




346

Figure 23. Thermal Coordinate Grid for Phase Angle $-15^{\circ} 28^{\prime}$ 


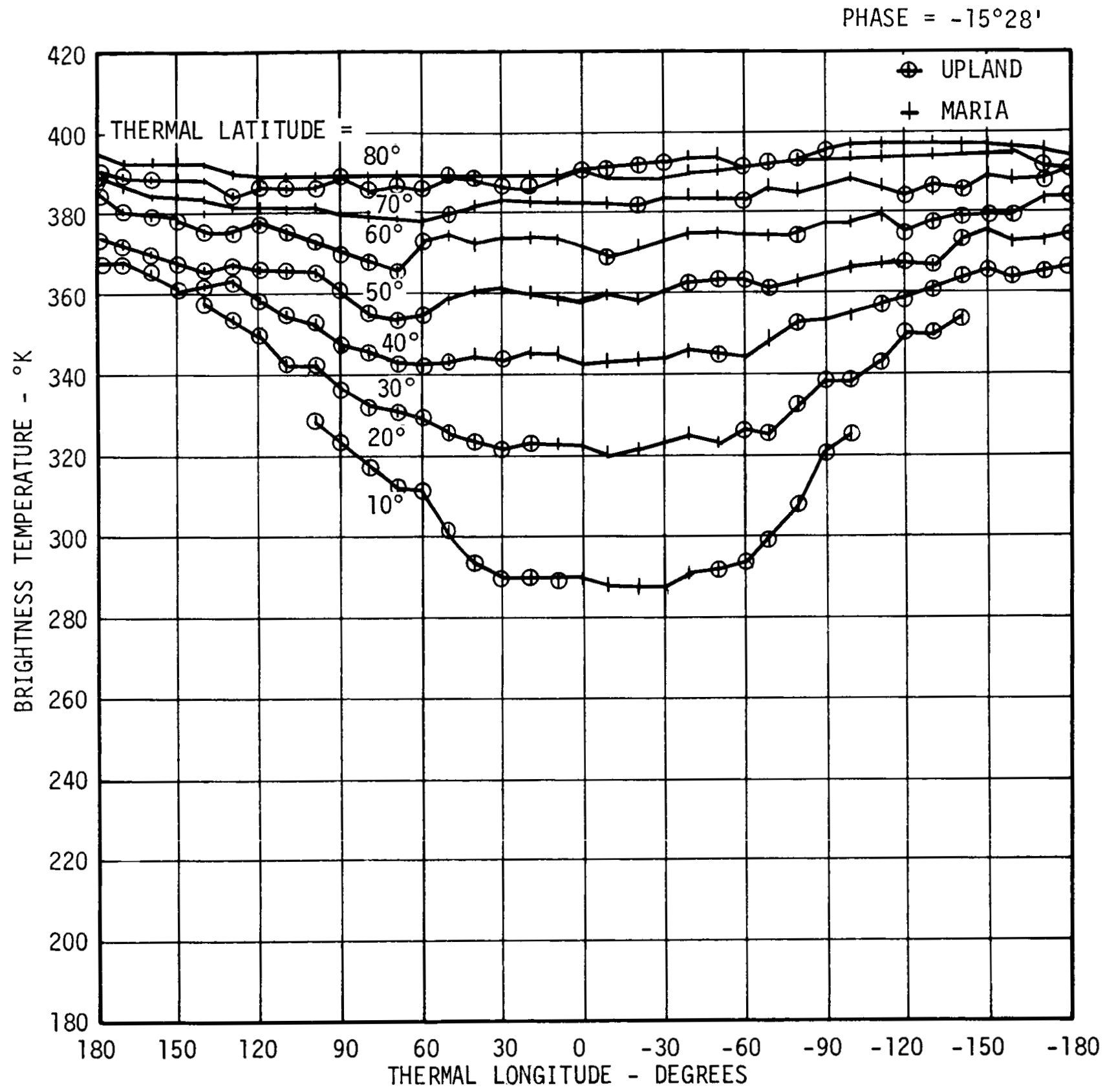

Figure 24. Brightness Temperature Versus Thermal Longitude at Phase $-15^{\circ} 28^{\prime}$ 


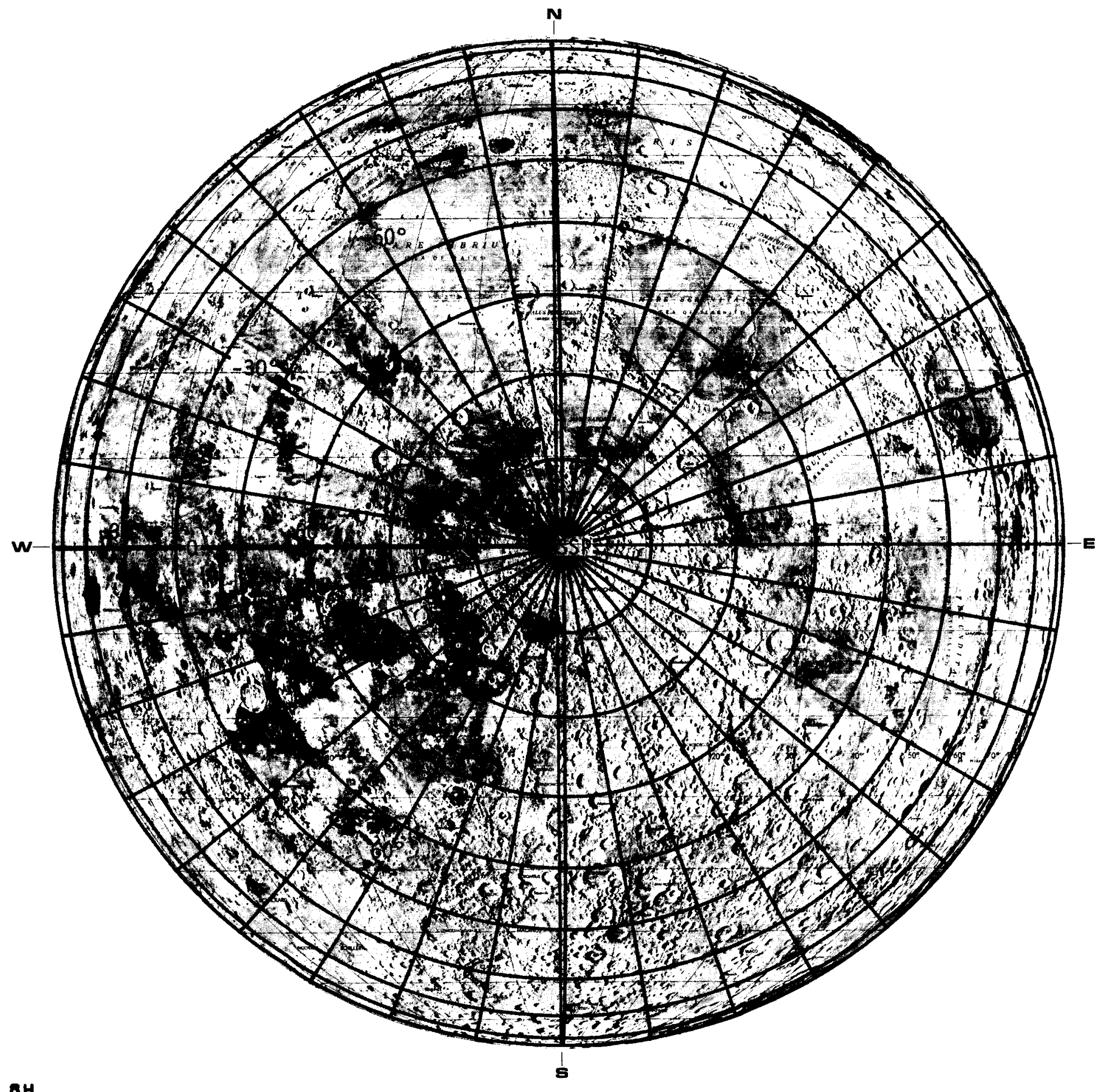

8 H

Figure 25. Thermal Coordinate Grid for Phase Angle $-2^{\circ} 16^{\prime}$ 


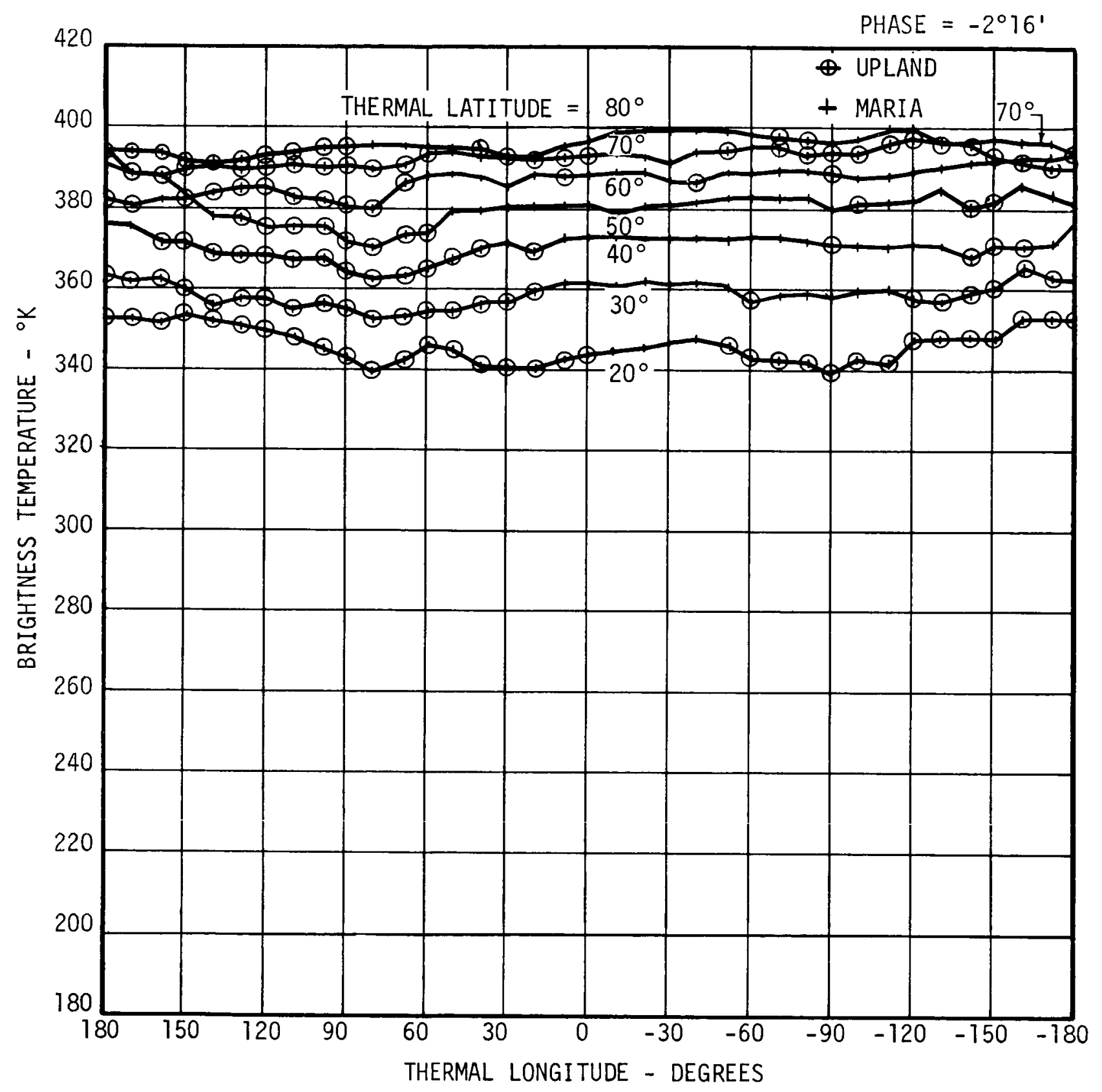

Figure 26. Brightness Temperature Versus Thermal Longitude at Phase $-2^{\circ} 16^{\prime}$ 


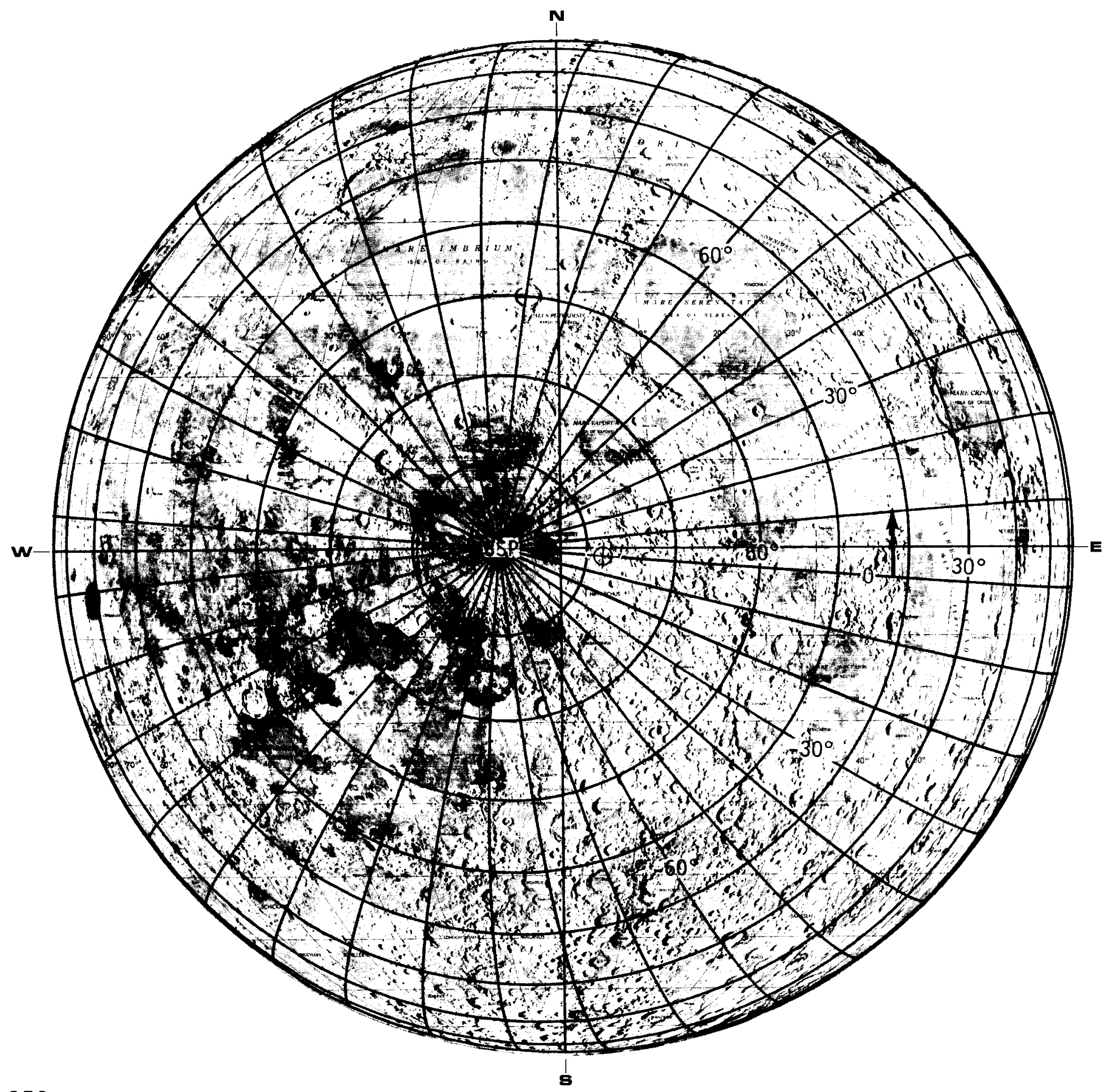

Figure 27. Thermal Coordinate Grid for Phase Angle $+11^{\circ} 42^{\prime}$ 


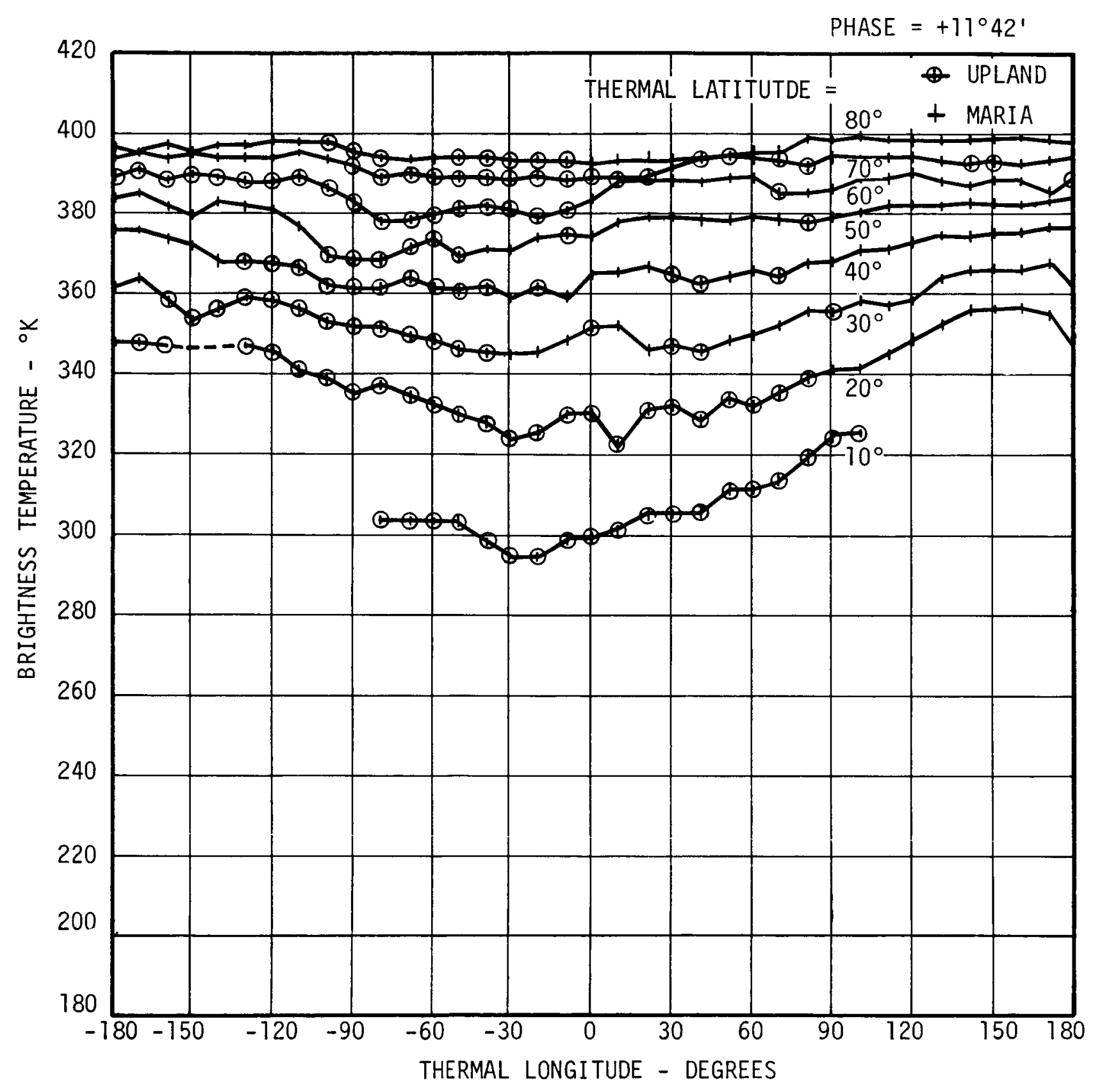

Figure 28. Brightness Temperature Versus Thermal Longitude at Phase $+11^{\circ} 42^{\prime}$ 


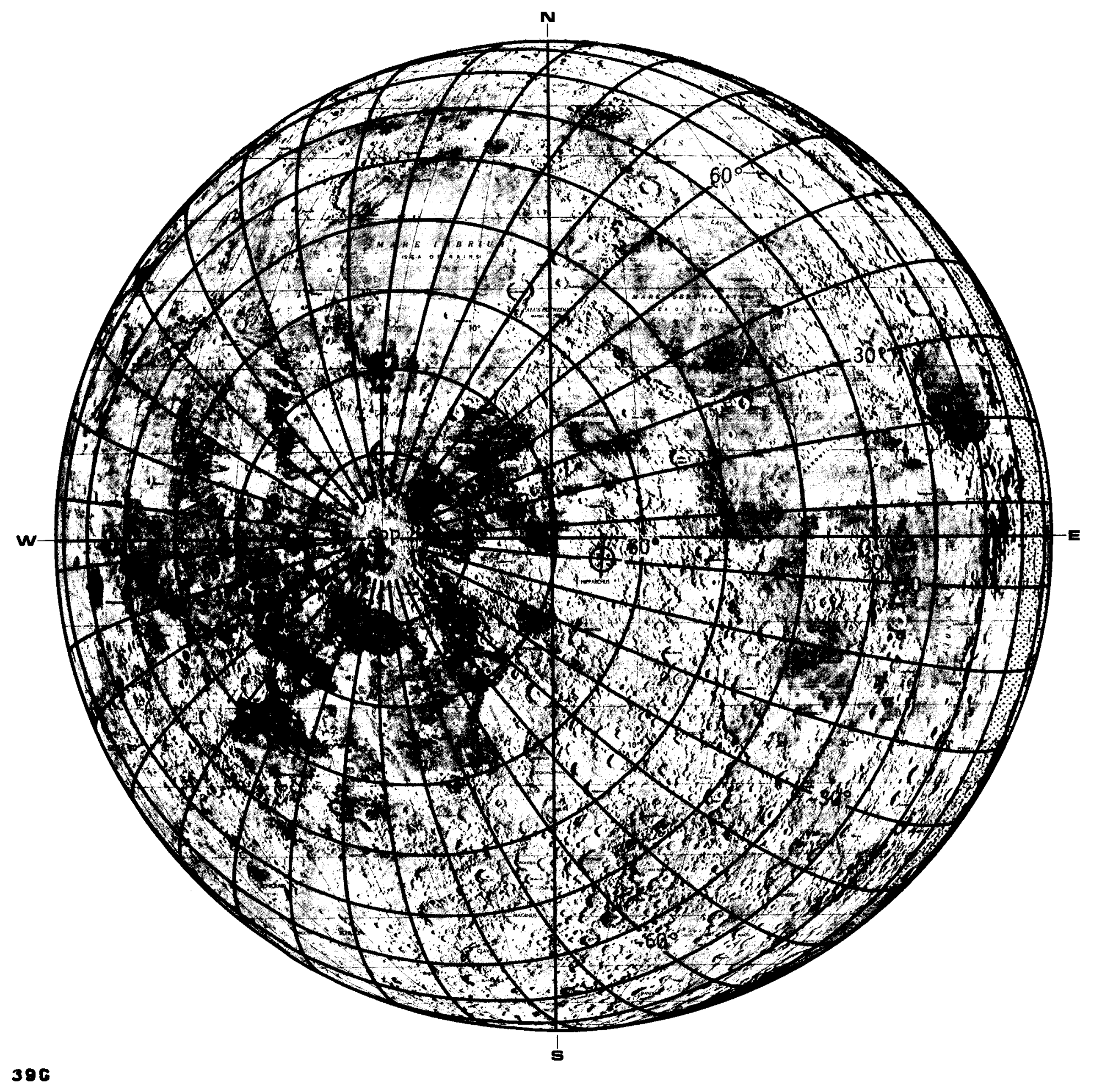

Figure 29. Thermal Coordinate Grid for Phase Angle $+25^{\circ} 37^{\prime}$ 


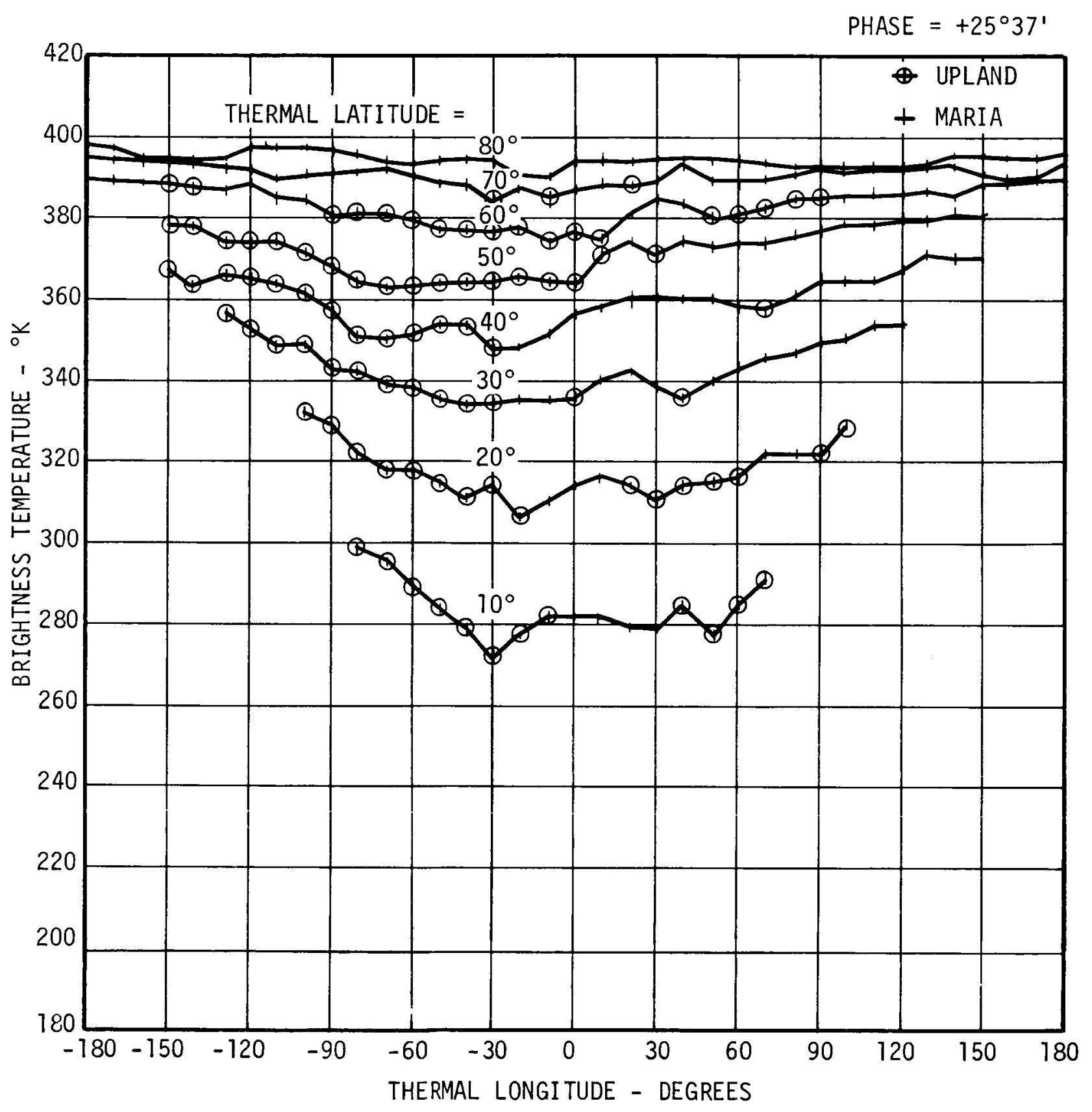

Figure 30. Brightness Temperature Versus Thermal Longitude at Phase $+25^{\circ} 37^{\prime}$ 


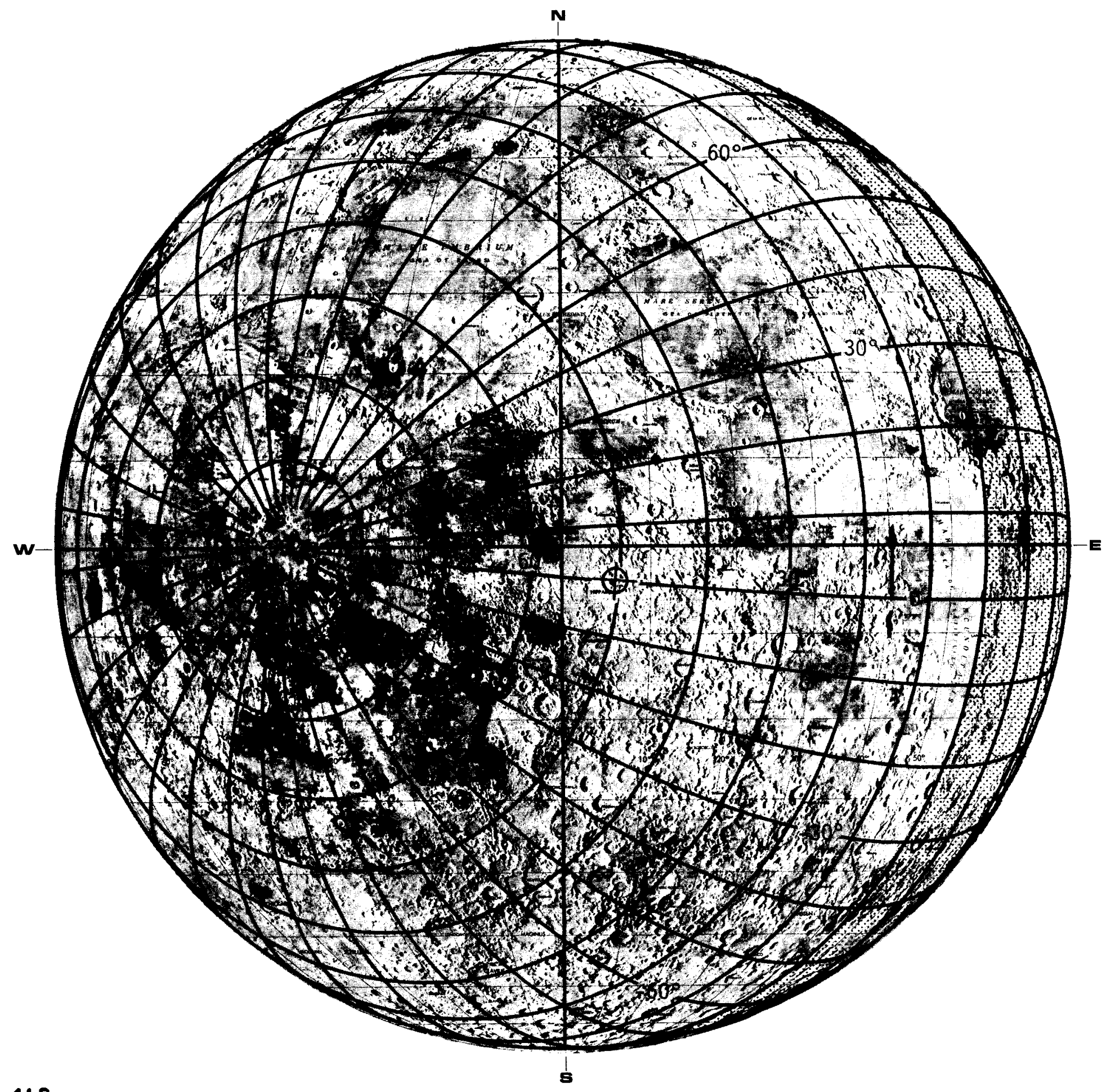

416

Figure 31. Thermal Coordinate Grid for Phase Angle $+39^{\circ} 51^{\prime}$ 


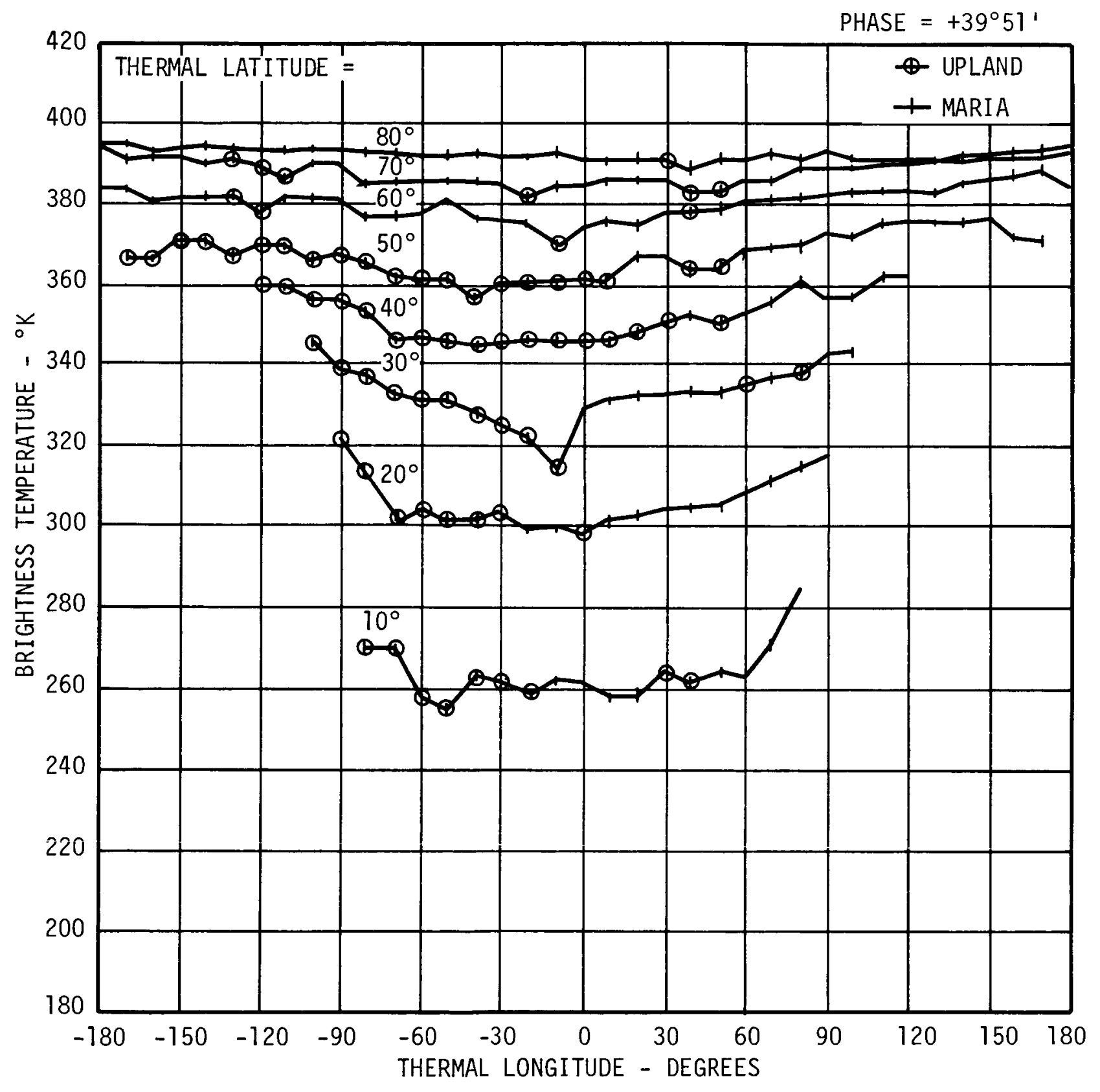

Figure 32. Brightness Temperature Versus Thermal Longitude at Phase $+39^{\circ} 51^{\prime}$ 


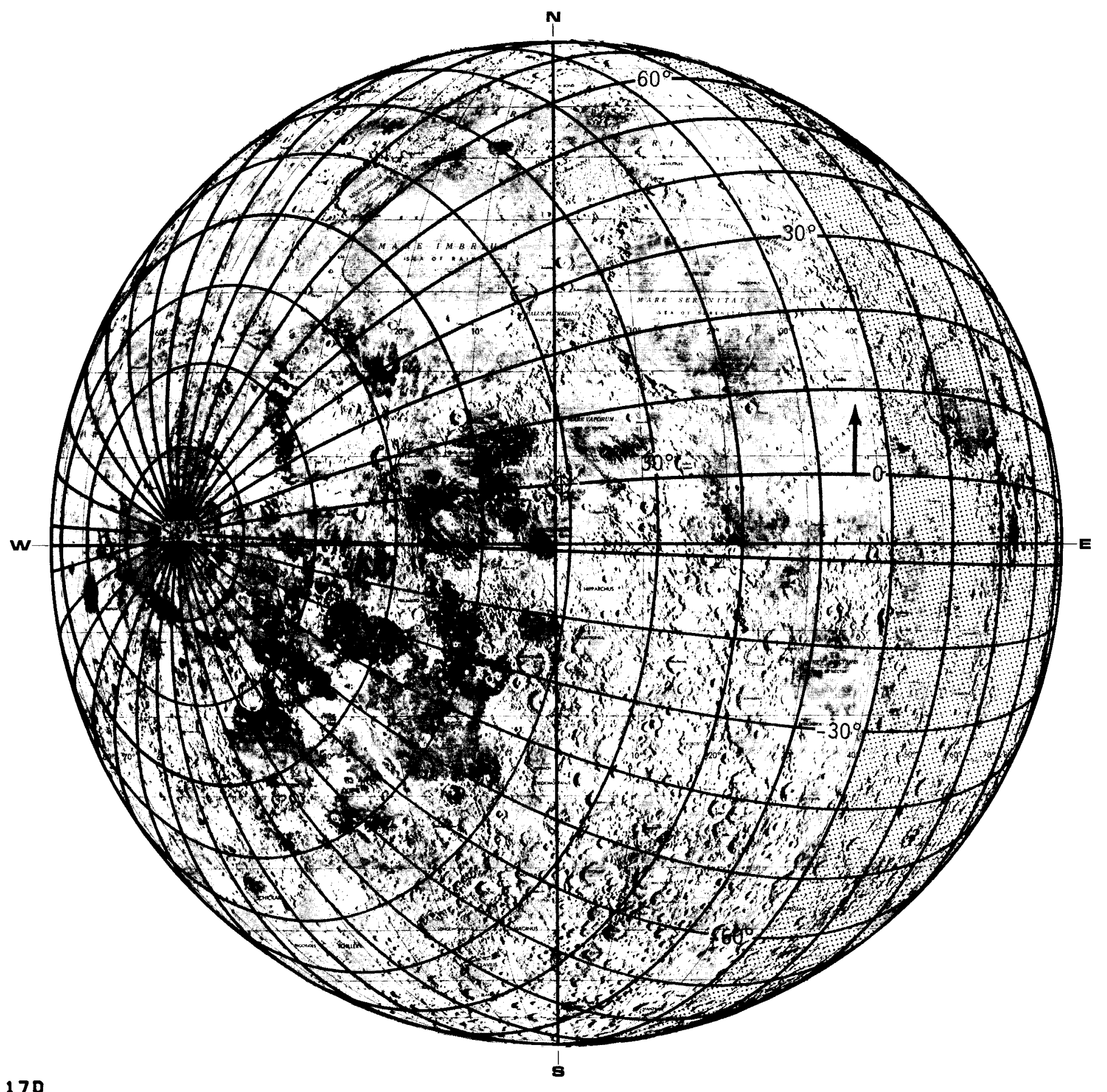

Figure 33. Thermal Coordinate Grid for Phase Angle $+49^{\circ} 12^{\prime}$ 


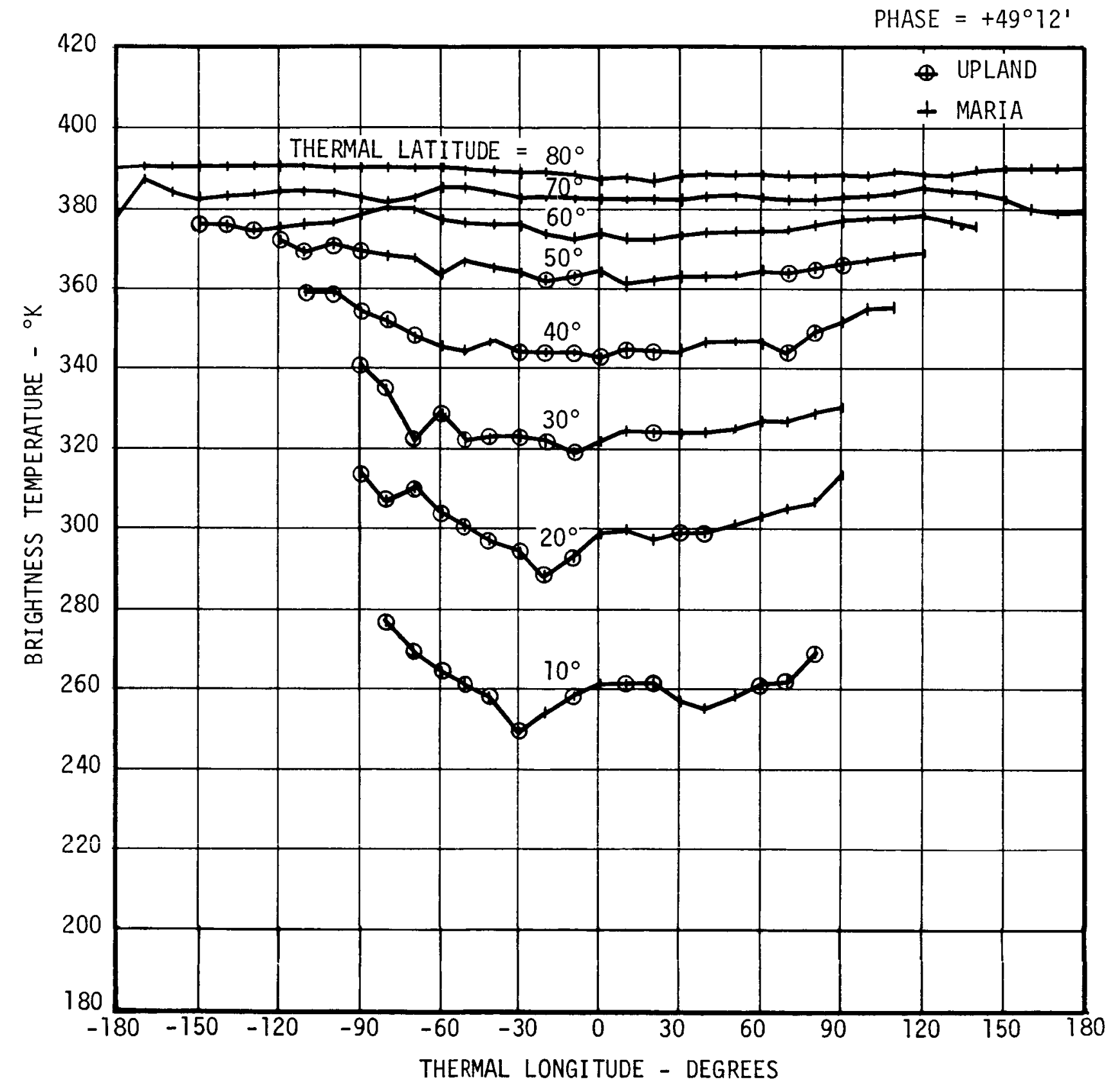

Figure 34. Brightness Temperature Versus Thermal Longitude at Phase $+49^{\circ} 12^{\prime}$ 


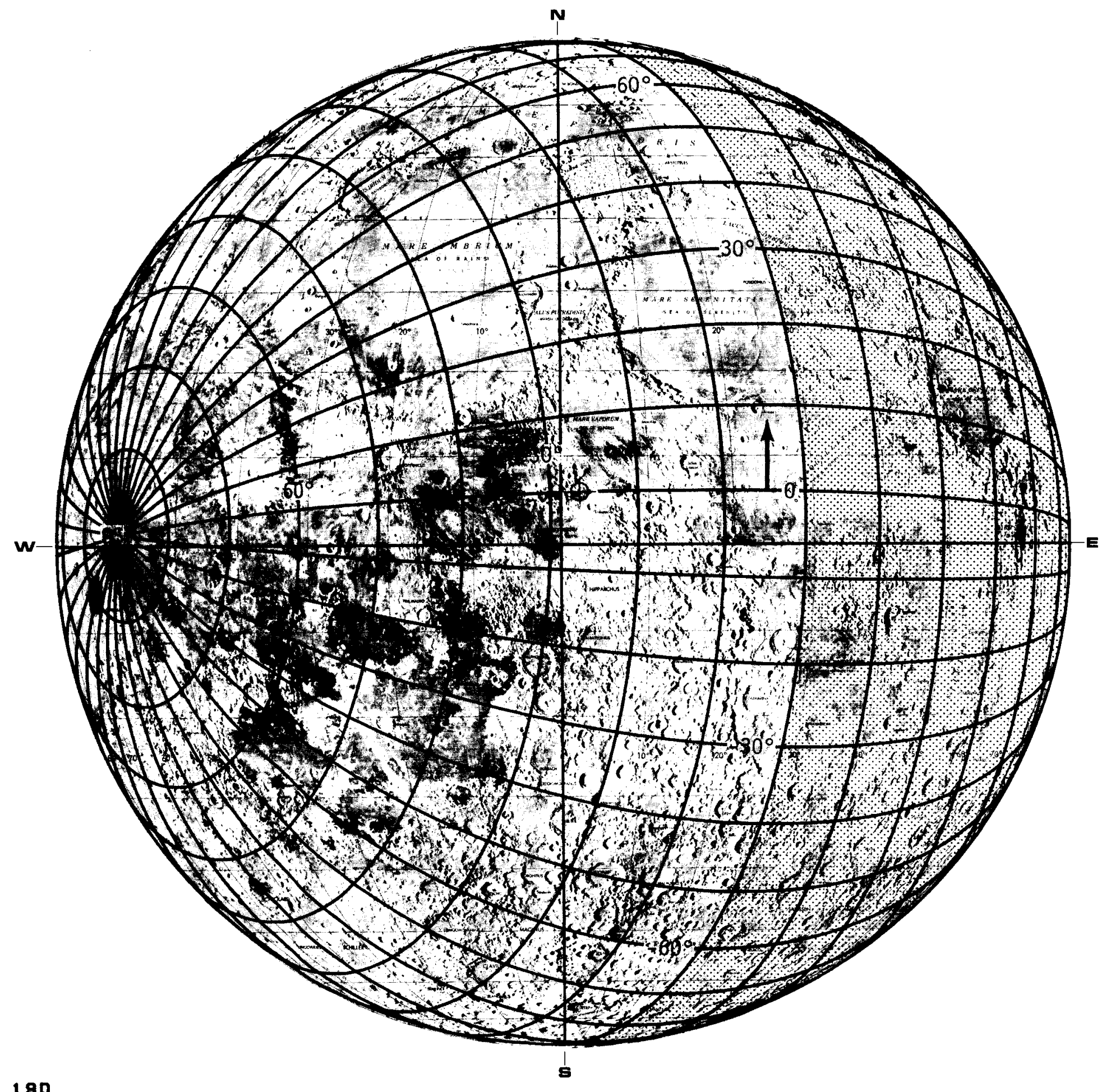

Figure 35. Thermal Coordinate Grid for Phase Angle $+63^{\circ} 23^{\prime}$ 


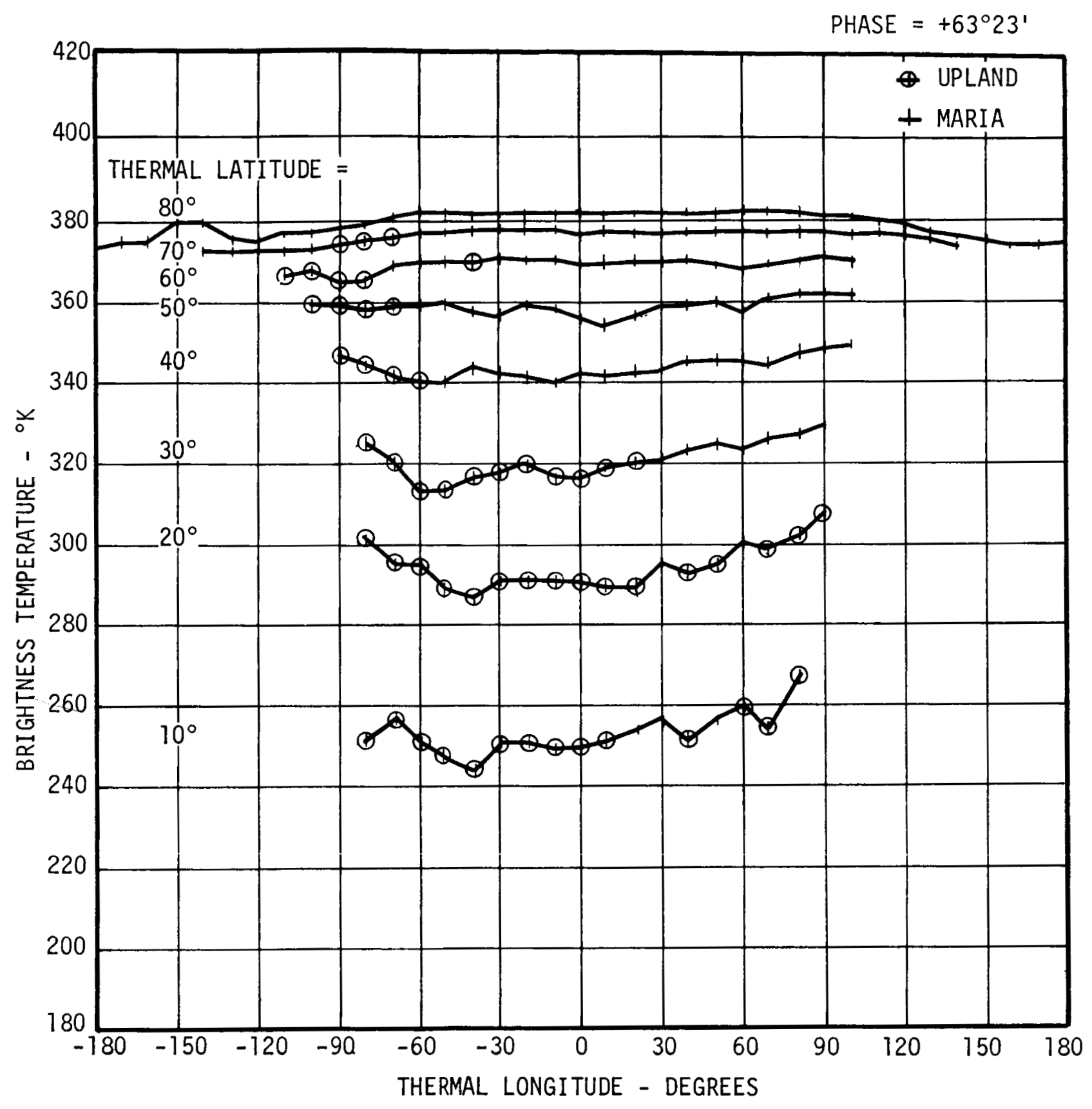

Figure 36. Brightness Temperature Versus Thermal Longitude at Phase $+63^{\circ} 23^{\prime}$ 


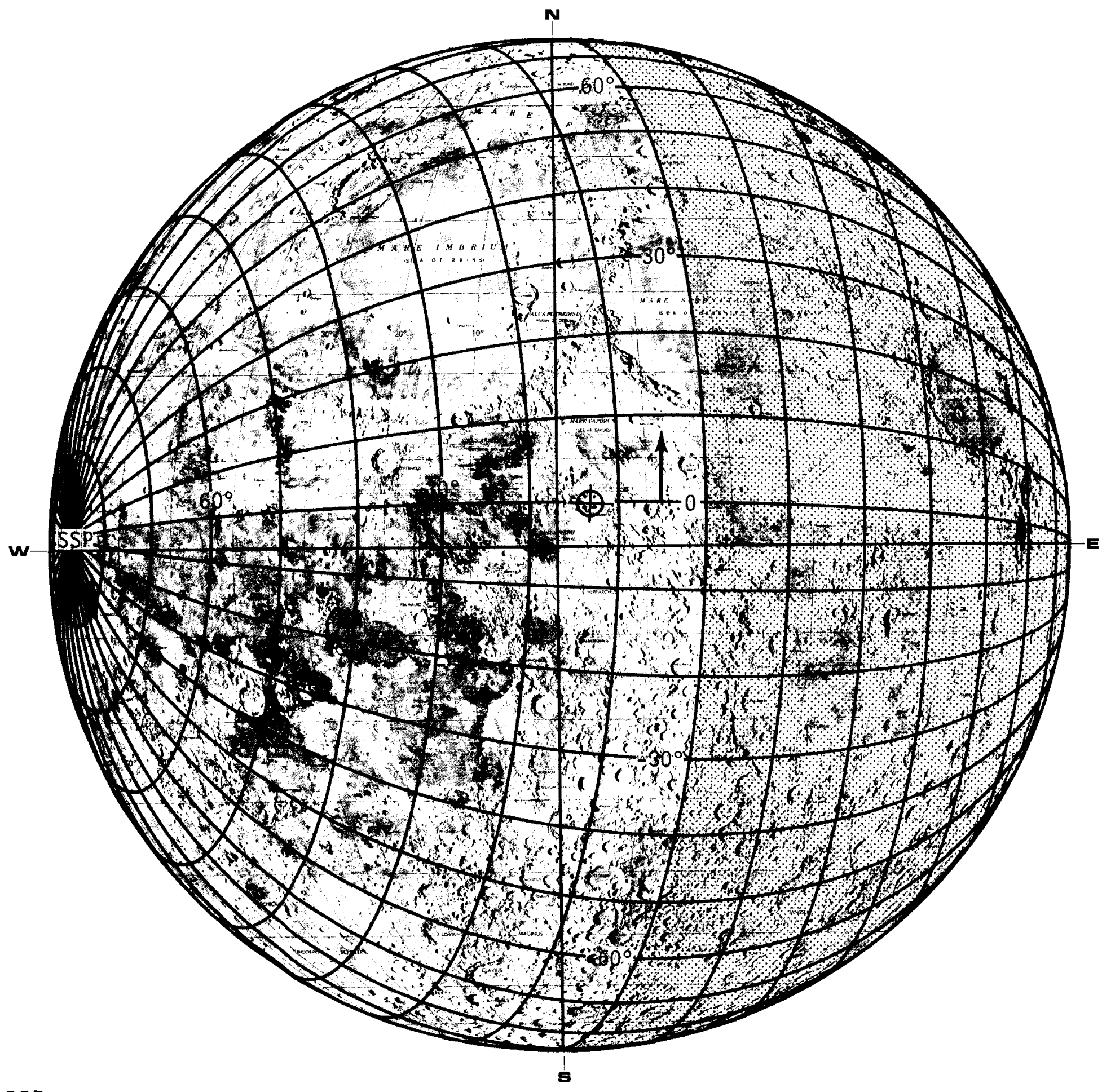

200

Figure 37. Thermal Coordinate Grid for Phase Angle $+76^{\circ} 42^{\prime}$ 


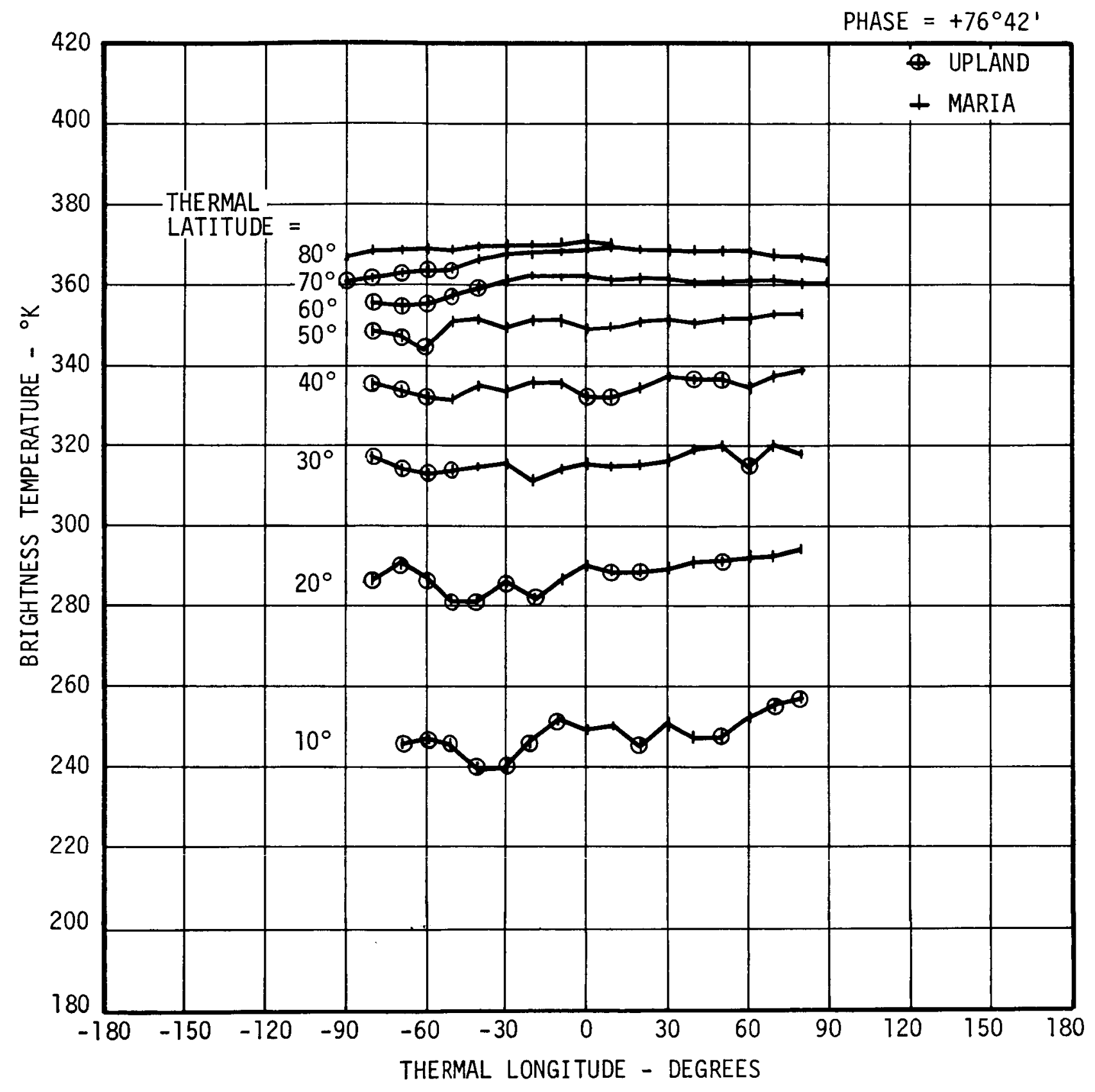

Figure 38. Brightness Temperature Versus Thermal Longitude at Phase $+76^{\circ} 42^{\prime}$ 


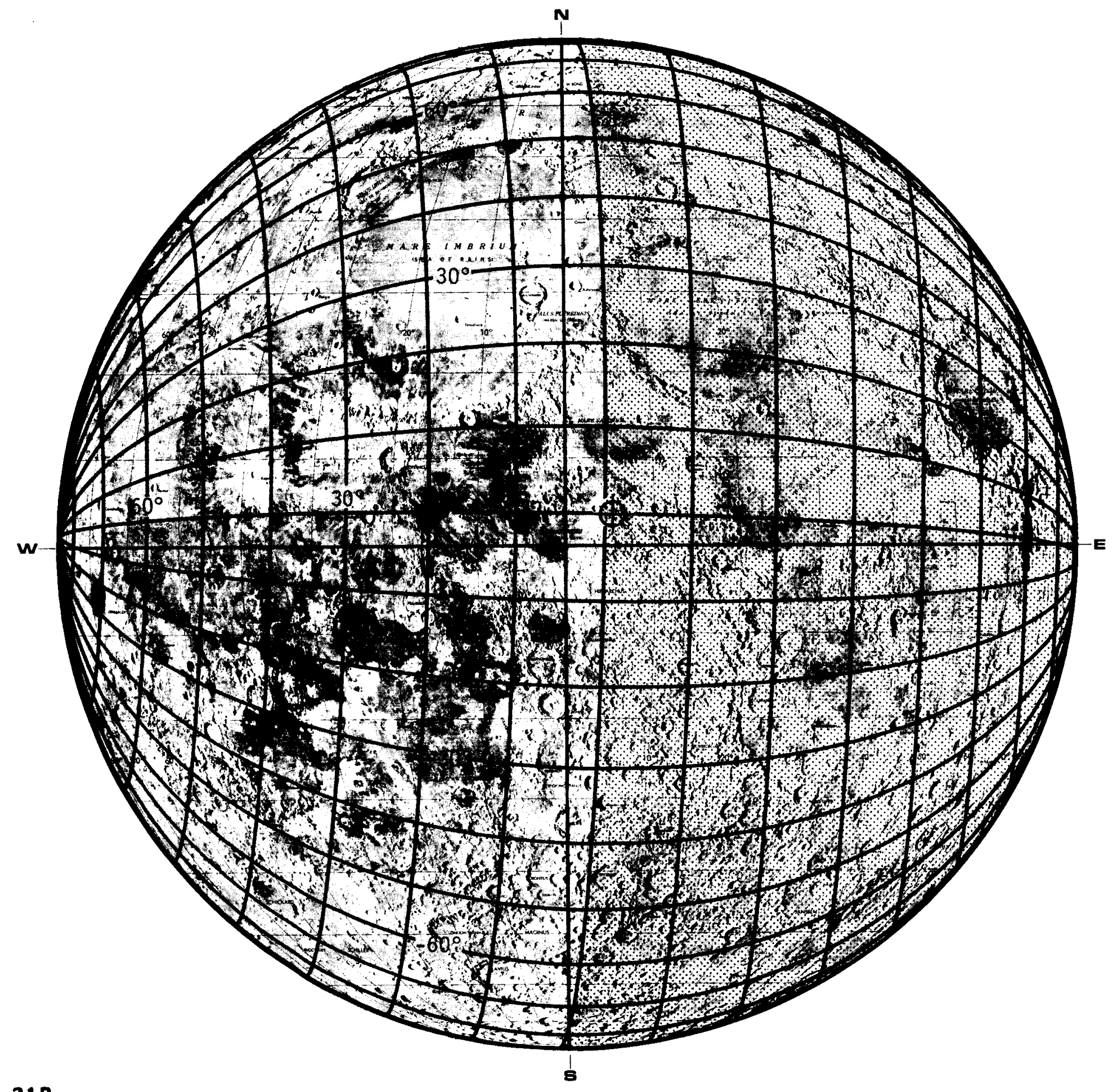

210

Figure 39. Thermal Coordinate Grid for Phase Angle $+90^{\circ} 16^{\prime}$ 
PHASE $=+90^{\circ} 16^{\prime}$

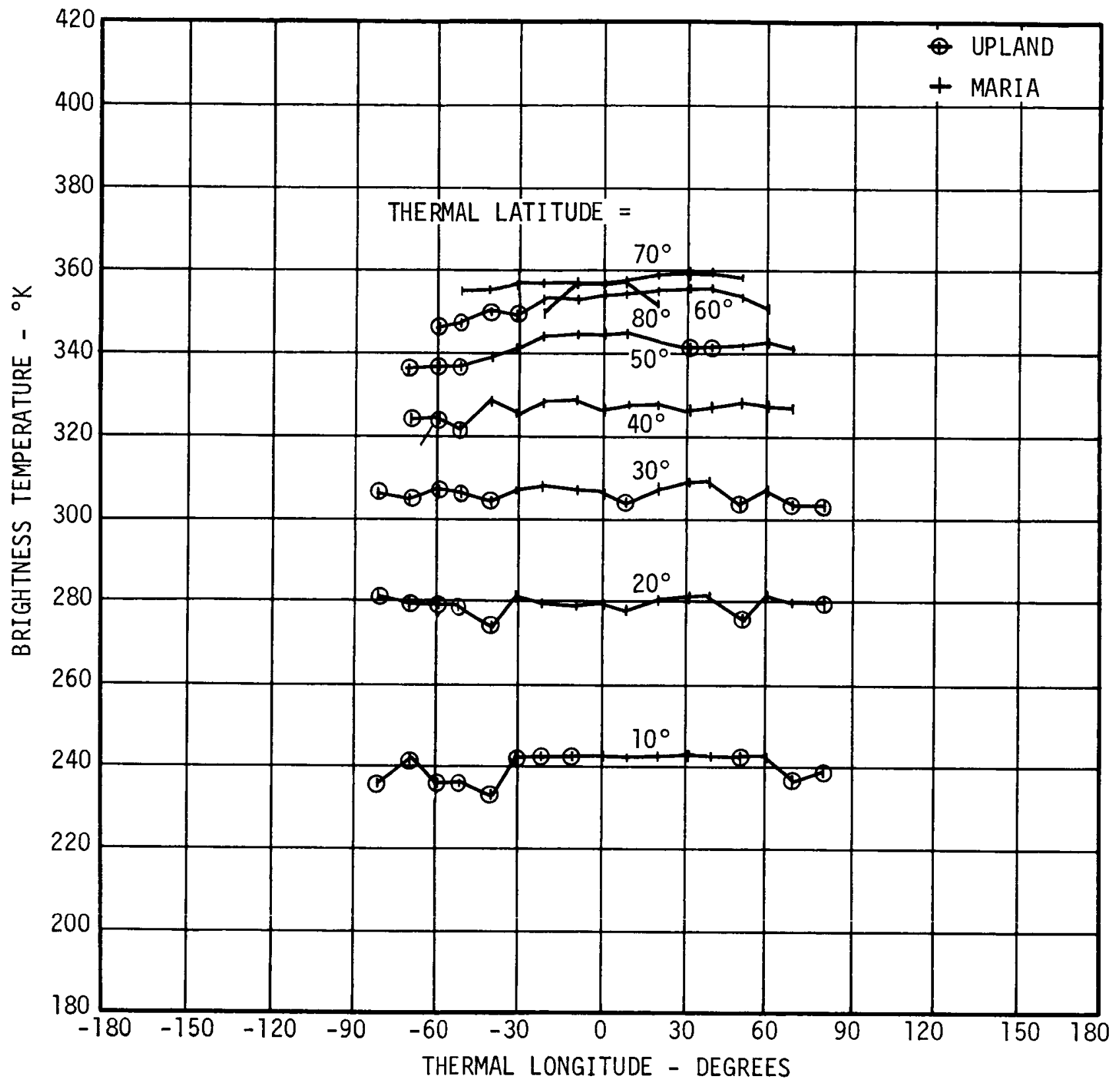

Figure 40. Brightness Temperature Versus Thermal Longitude at Phase $+90^{\circ} 16^{\prime}$ 


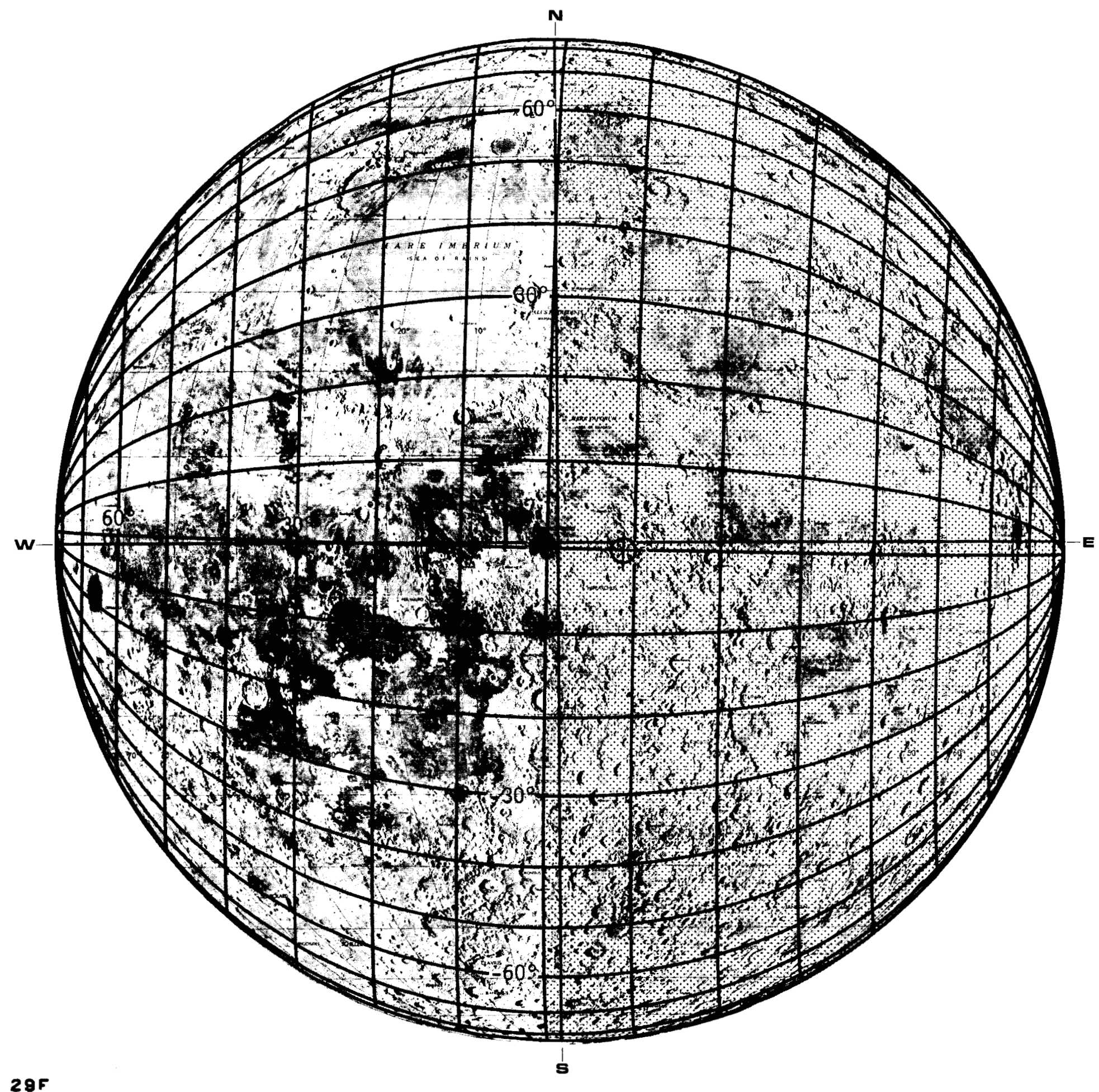

Figure 41. Thermal Coordinate Grid for Phase Angle $+98^{\circ} 40^{\prime}$ 


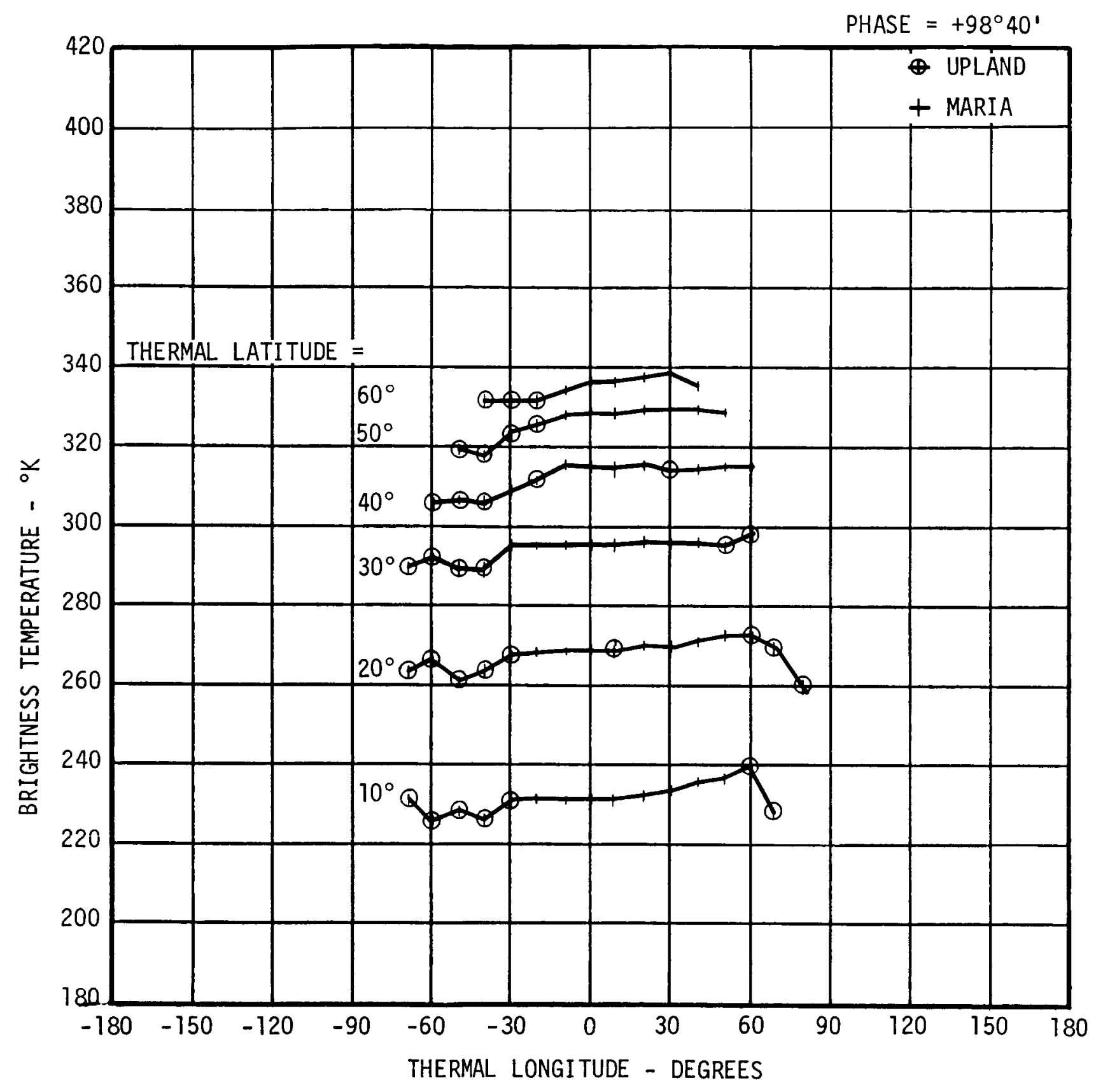

Figure 42. Brightness Temperature Versus Thermal Longitude at Phase $+98^{\circ} 40^{\prime}$ 


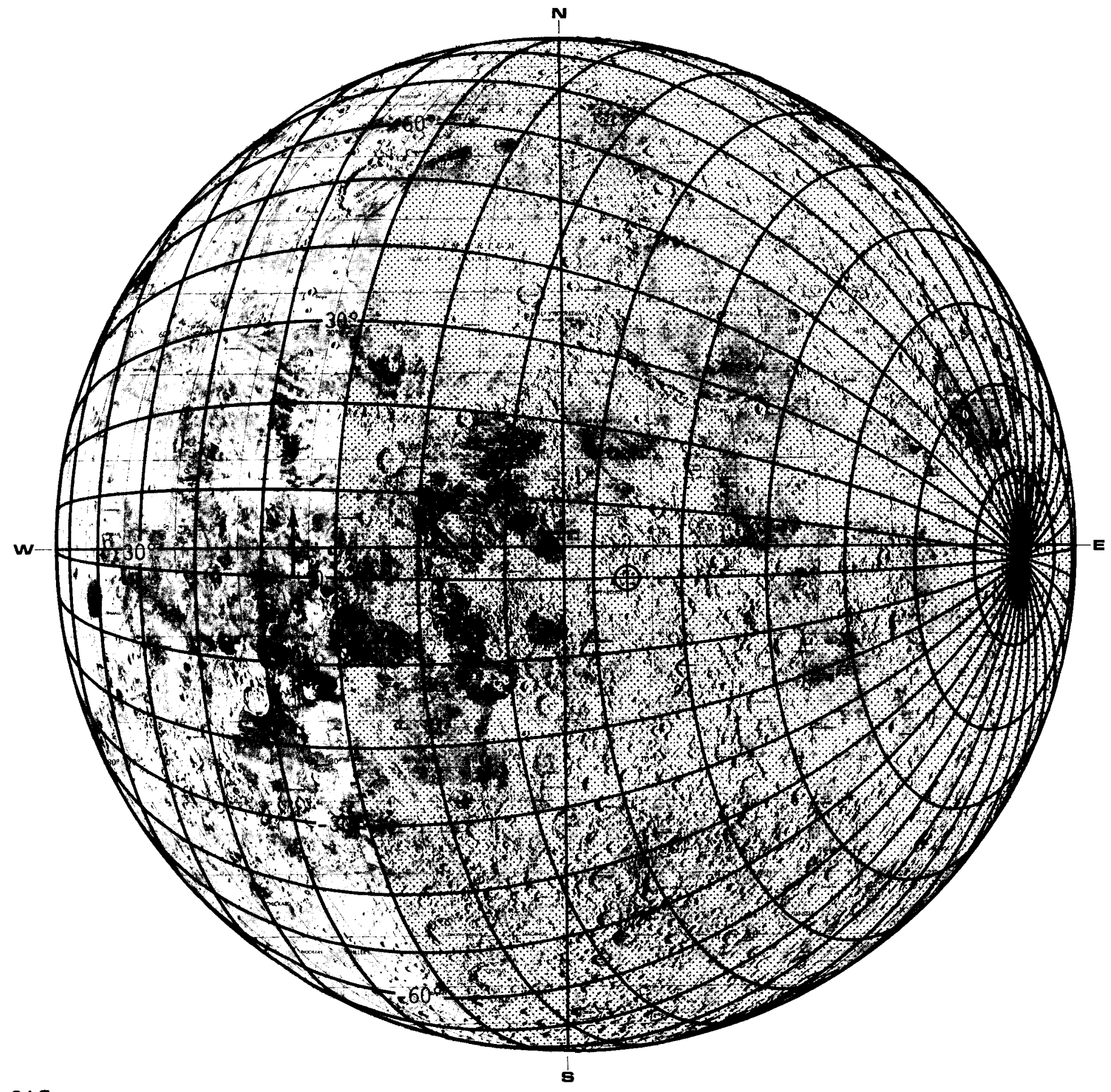

Figure 43. Thermal Coordinate Grid for Phase Angle $+123^{\circ} 51^{\prime}$ 


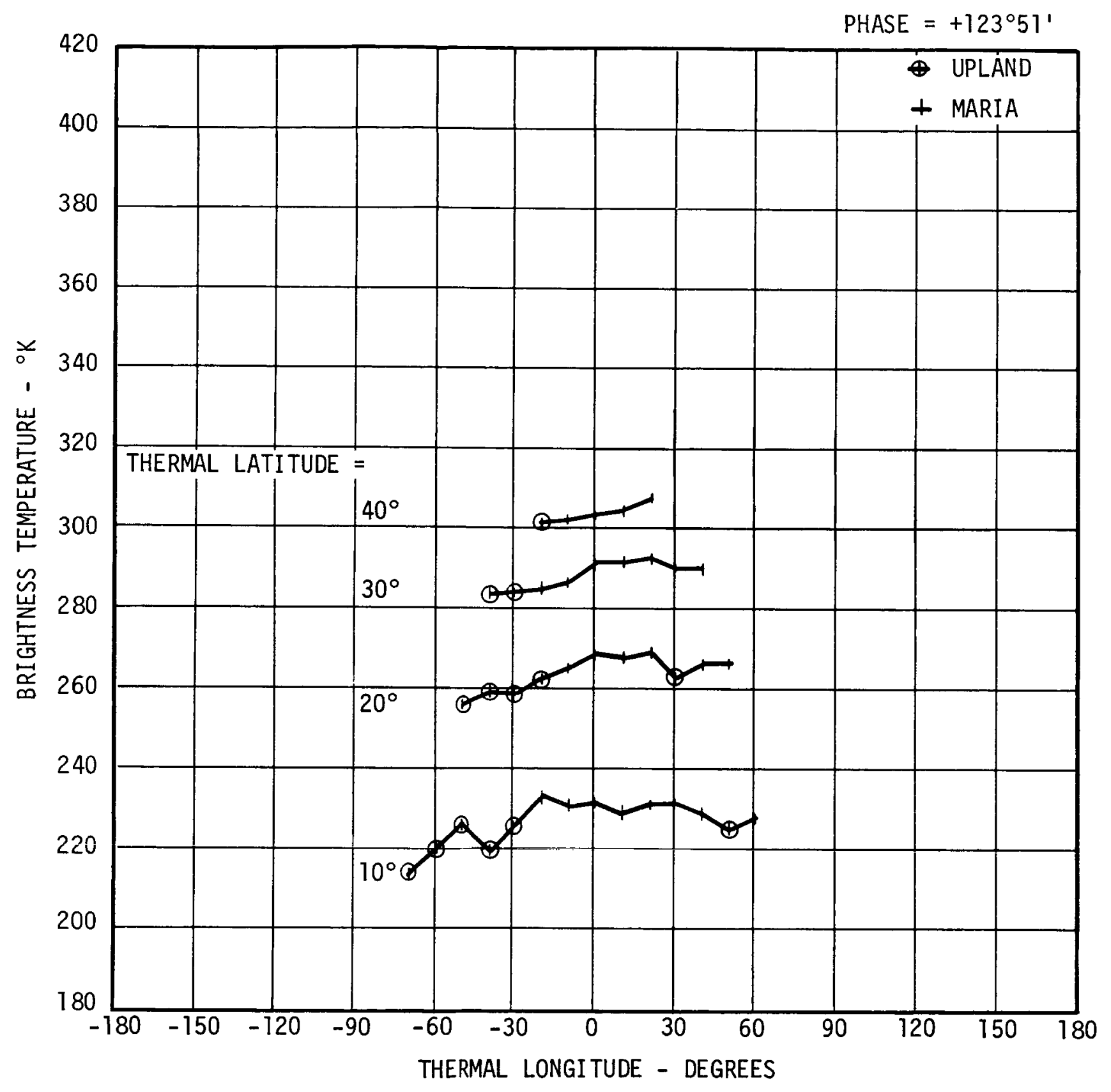

Figure 44. Brightness Temperature Versus Thermal Longitude at Phase $+123^{\circ} 51^{\prime}$ 




Figure 45. Thermal Coordinate Grid for Phase Angle $+135^{\circ} 40^{\prime}$ 


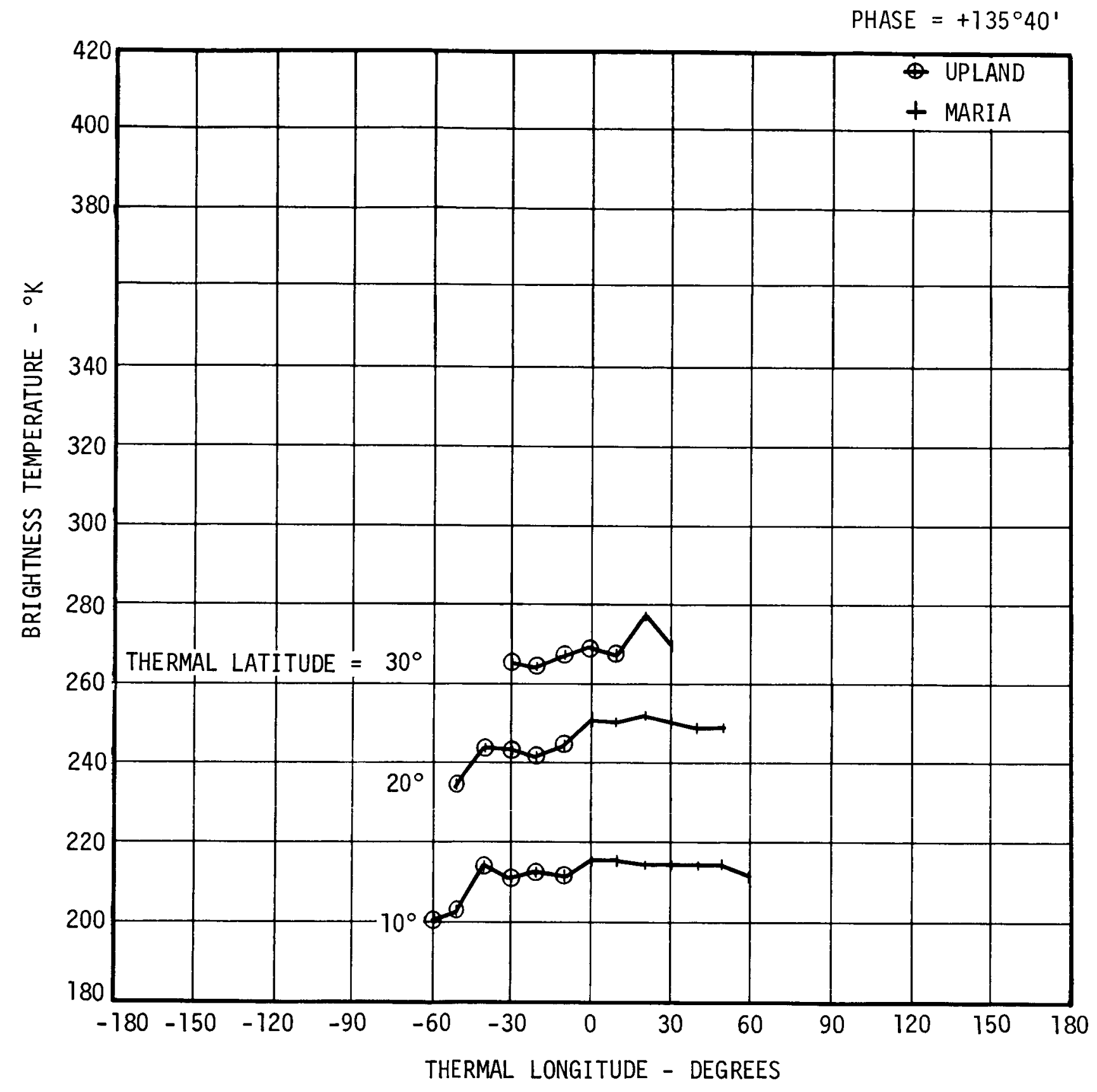

Figure 46. Brightness Temperature Versus Thermal Longitude at Phase $+135^{\circ} 40^{\prime}$ 


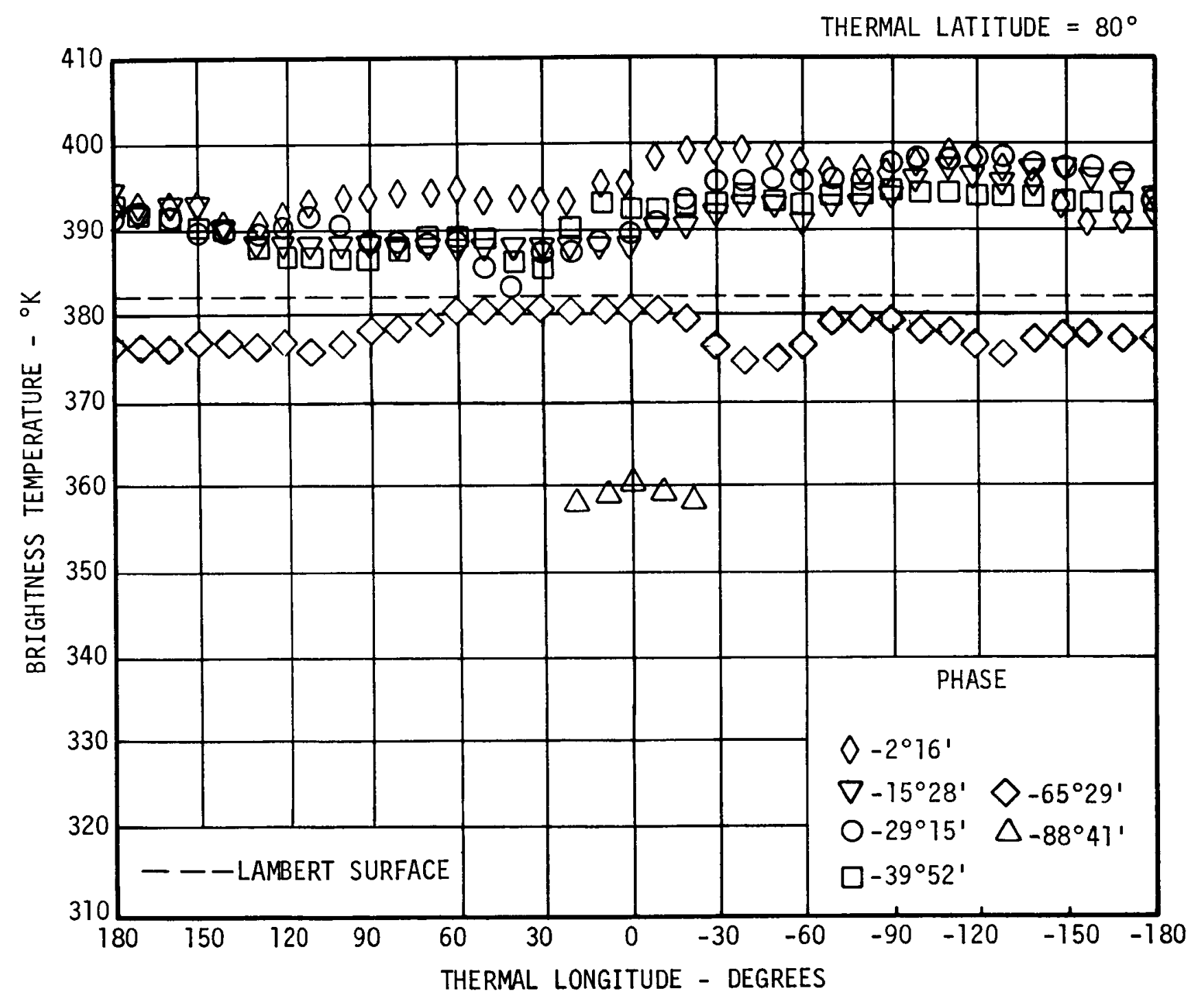

Figure 47. Brightness Temperature Versus Thermal Longitude for Negative Phases at Thermal Latitude $80^{\circ}$ 


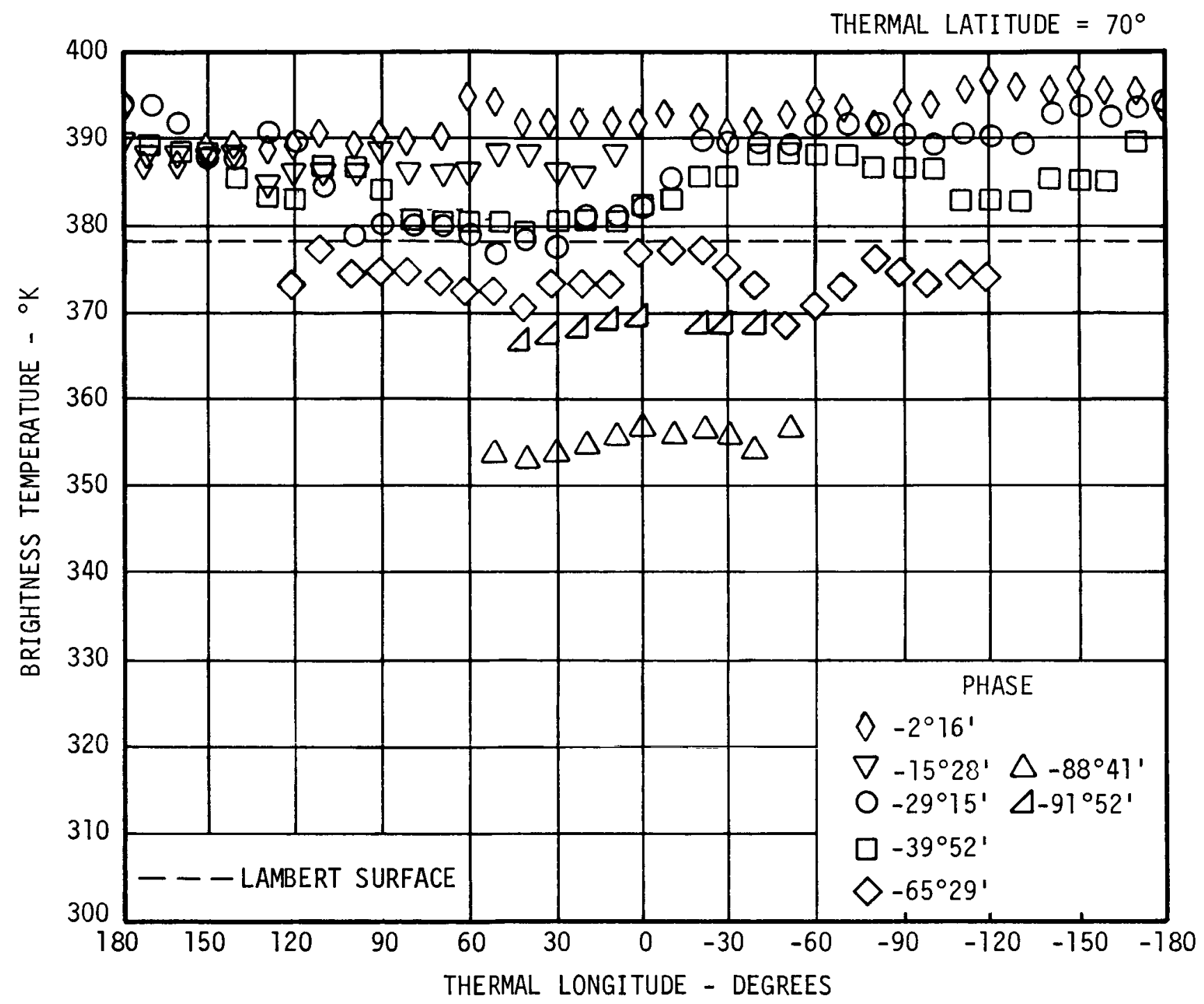

Figure 48. Brightness Temperature Versus Thermal Longitude for Negative Phases at Thermal Latitude $70^{\circ}$ 
THERMAL LATITUDE $=60^{\circ}$

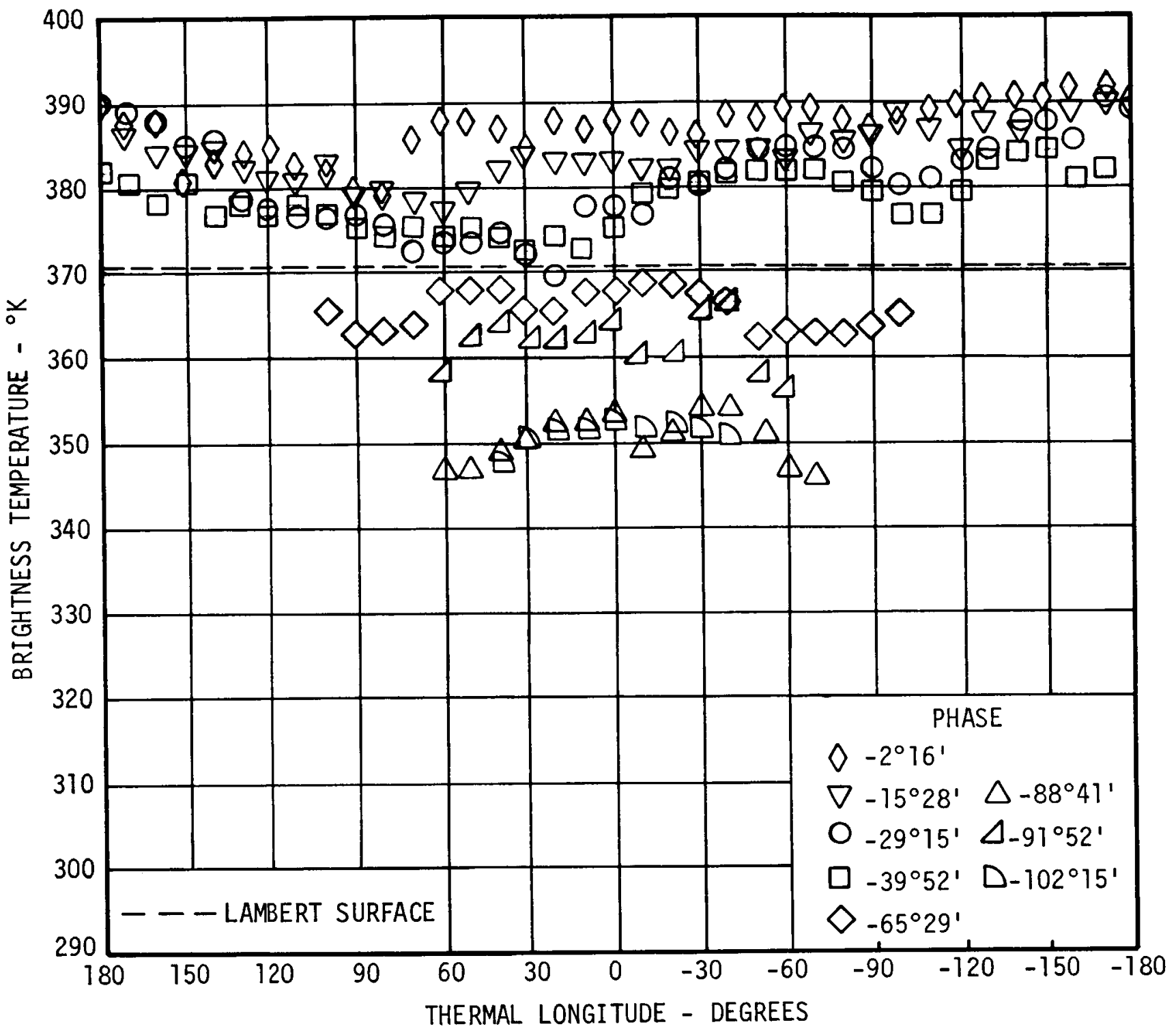

Figure 49. Brightness Temperature Versus Thermal Longitude for Negative Phases at Thermal Latitude $60^{\circ}$ 


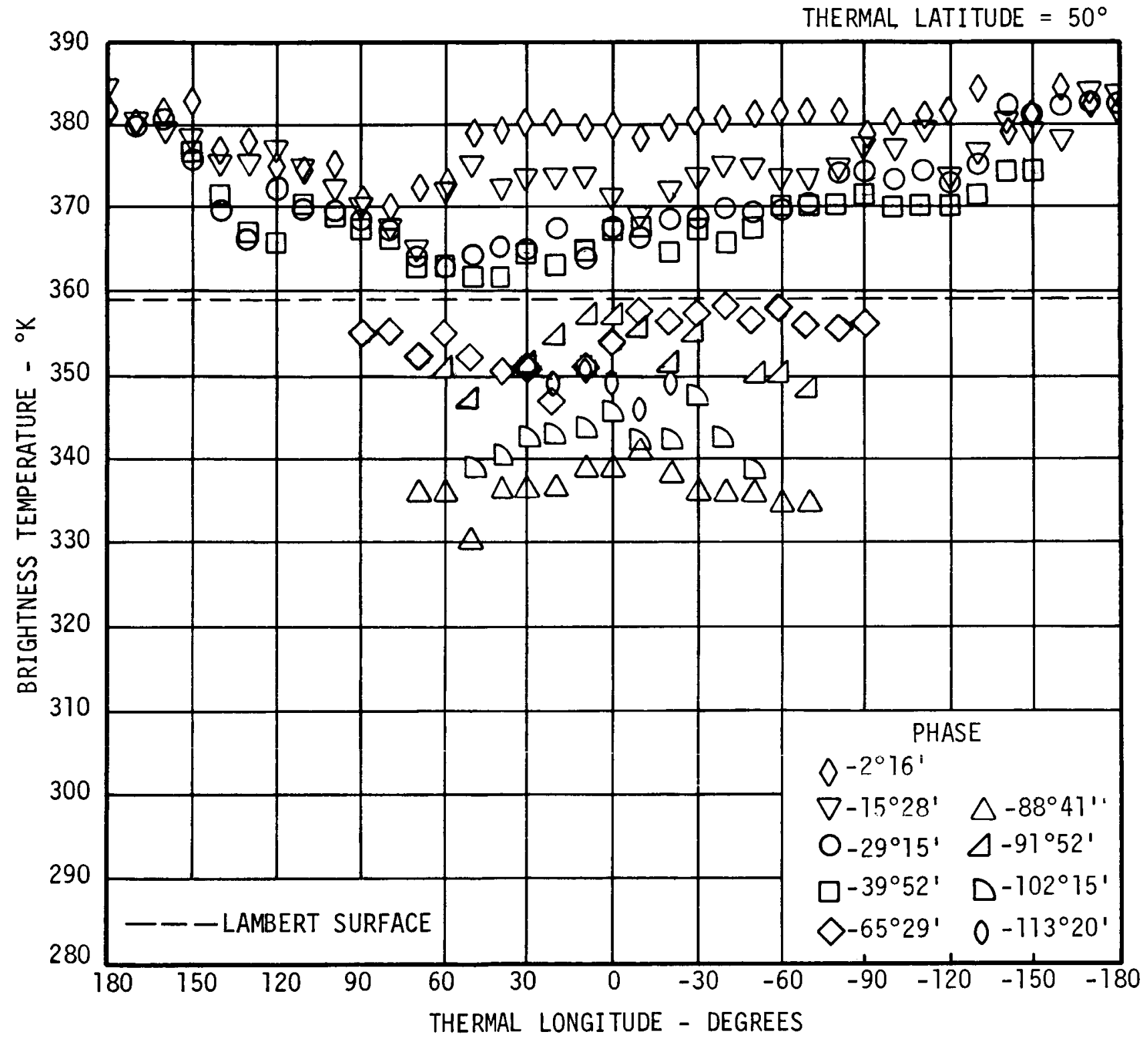

Figure 50. Brightness Temperature Versus Thermal Longitude for Negative Phases at Thermal Latitude $50^{\circ}$ 


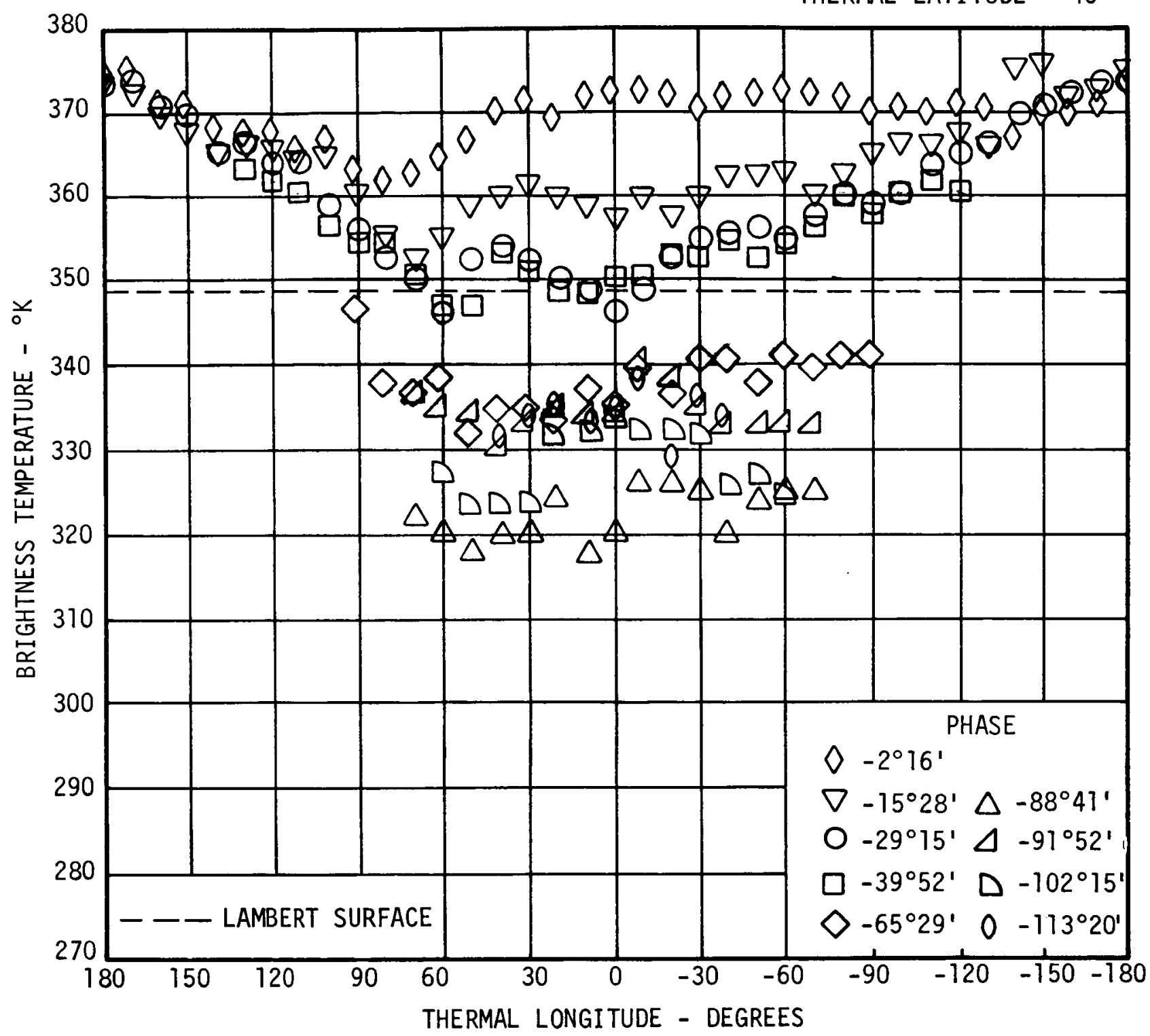

Figure 51. Brightness Temperature Versus Thermal Longitude for Negative Phases at Thermal Latitude $40^{\circ}$ 
THERMAL LATITUDE $=30^{\circ}$

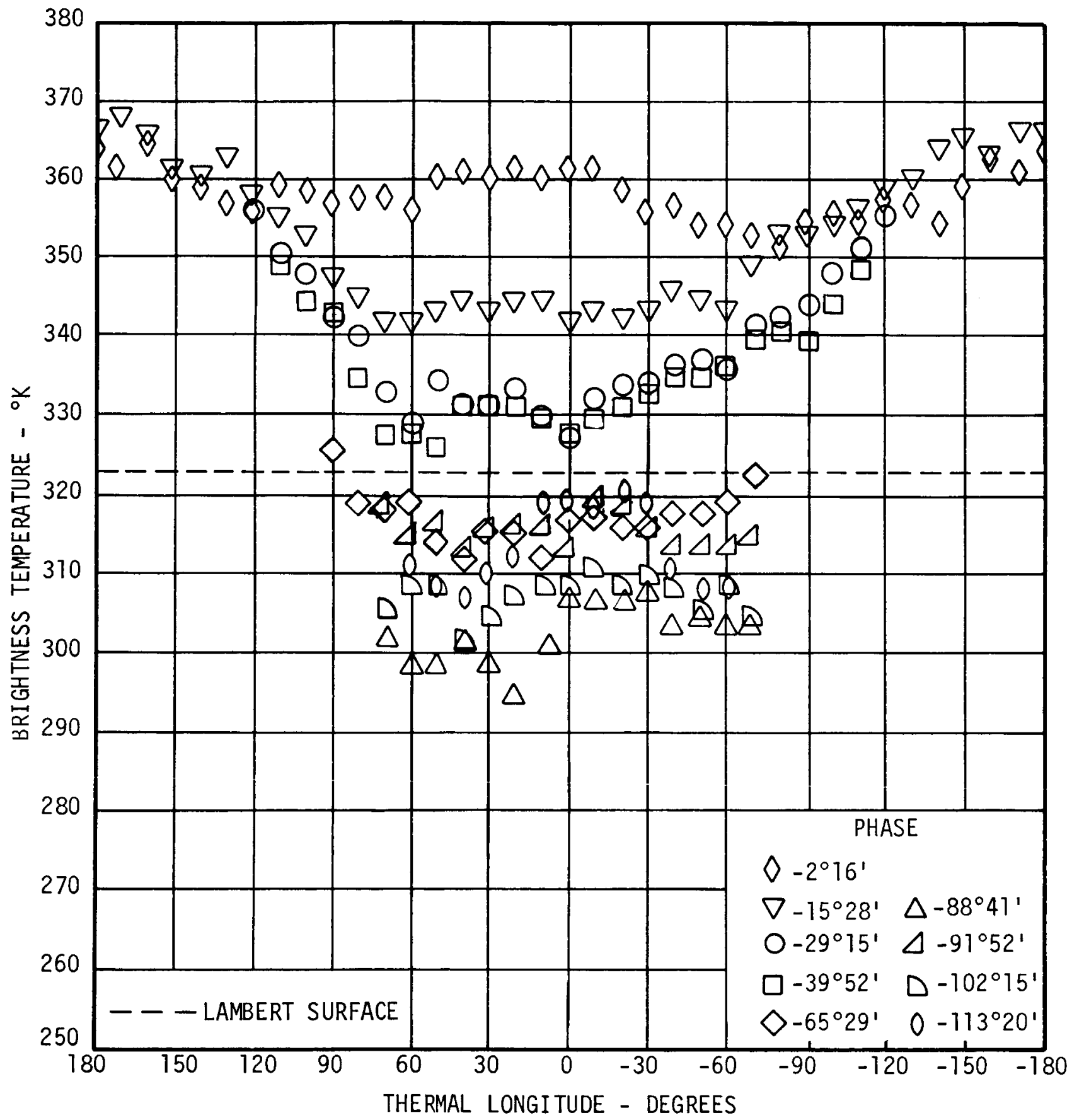

Figure 52. Brightness Temperature Versus Thermal Longitude for Negative Phases at Thermal Latitude $30^{\circ}$ 


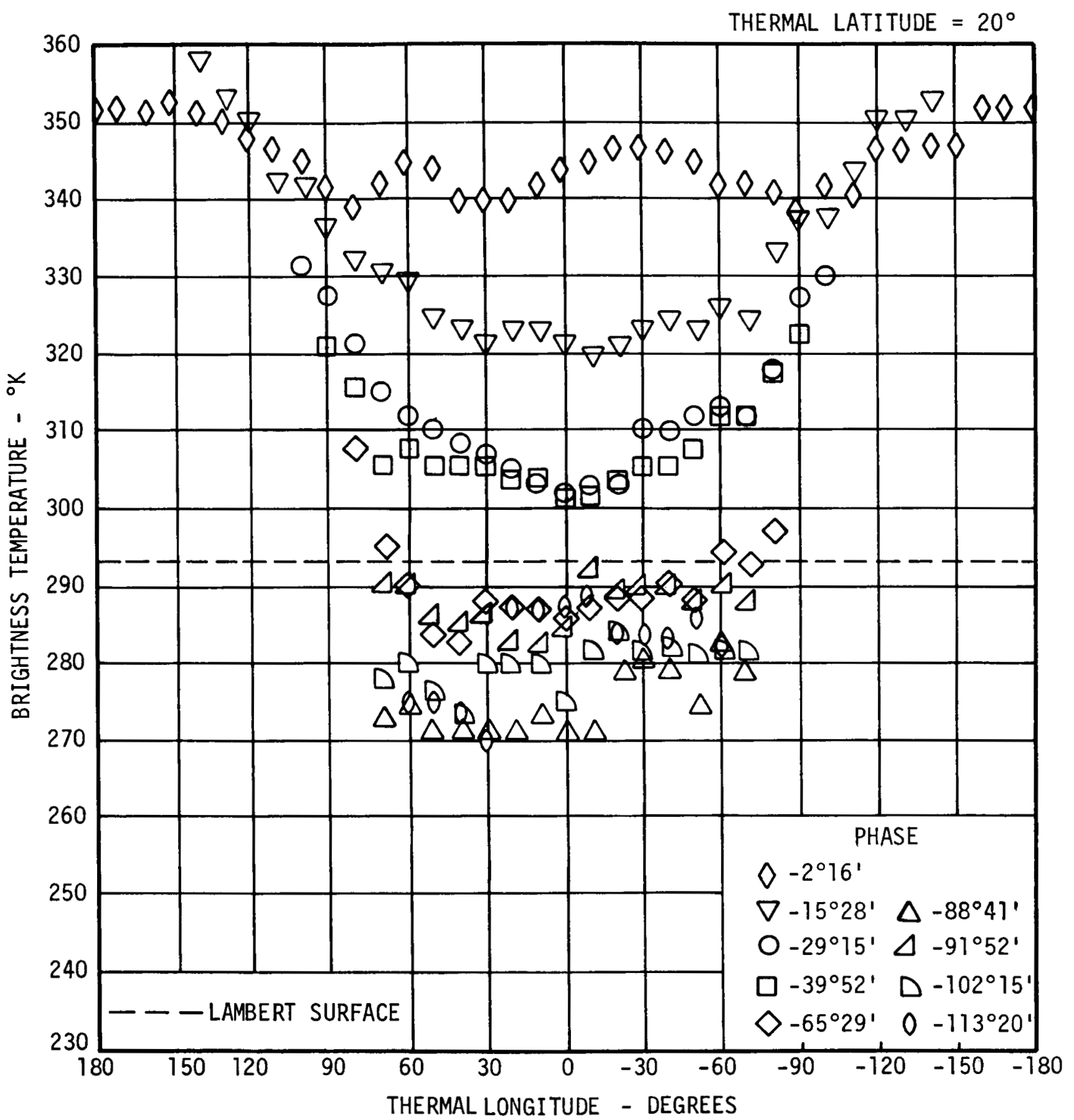

Figure 53. Brightness Temperature Versus Thermal Longitude for Negative Phases at Thermal Latitude $20^{\circ}$ 


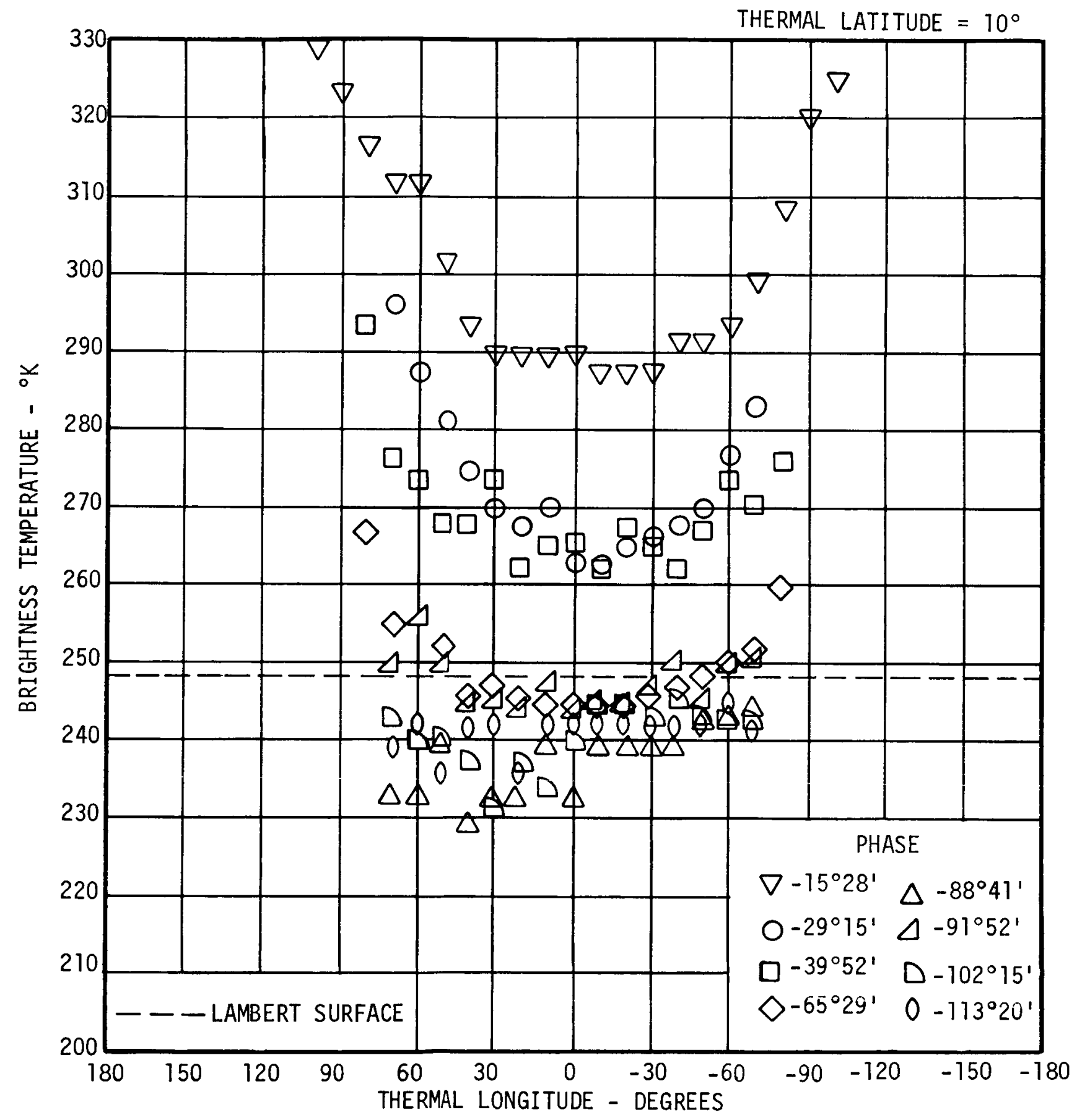

Figure 54. Brightness Temperature Versus Thermal Longitude for Negative Phases at Thermal Latitude $10^{\circ}$ 
THERMAL LATITUDE $=80^{\circ}$

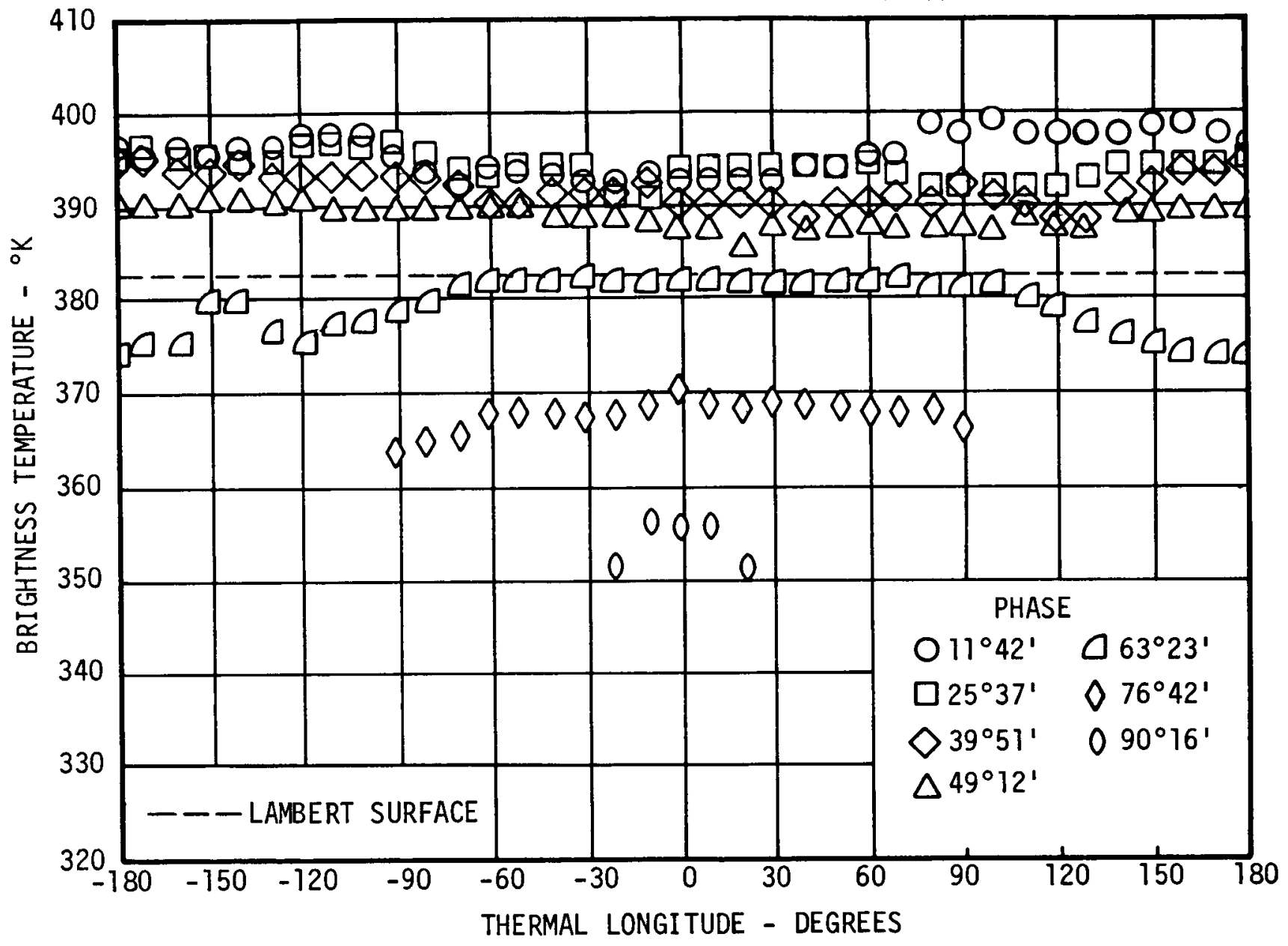

Figure 55. Brightness Temperature Versus Thermal Longitude for Positive Phases at Thermal Latitude $80^{\circ}$ 
THERMAL LATITUDE $=70^{\circ}$

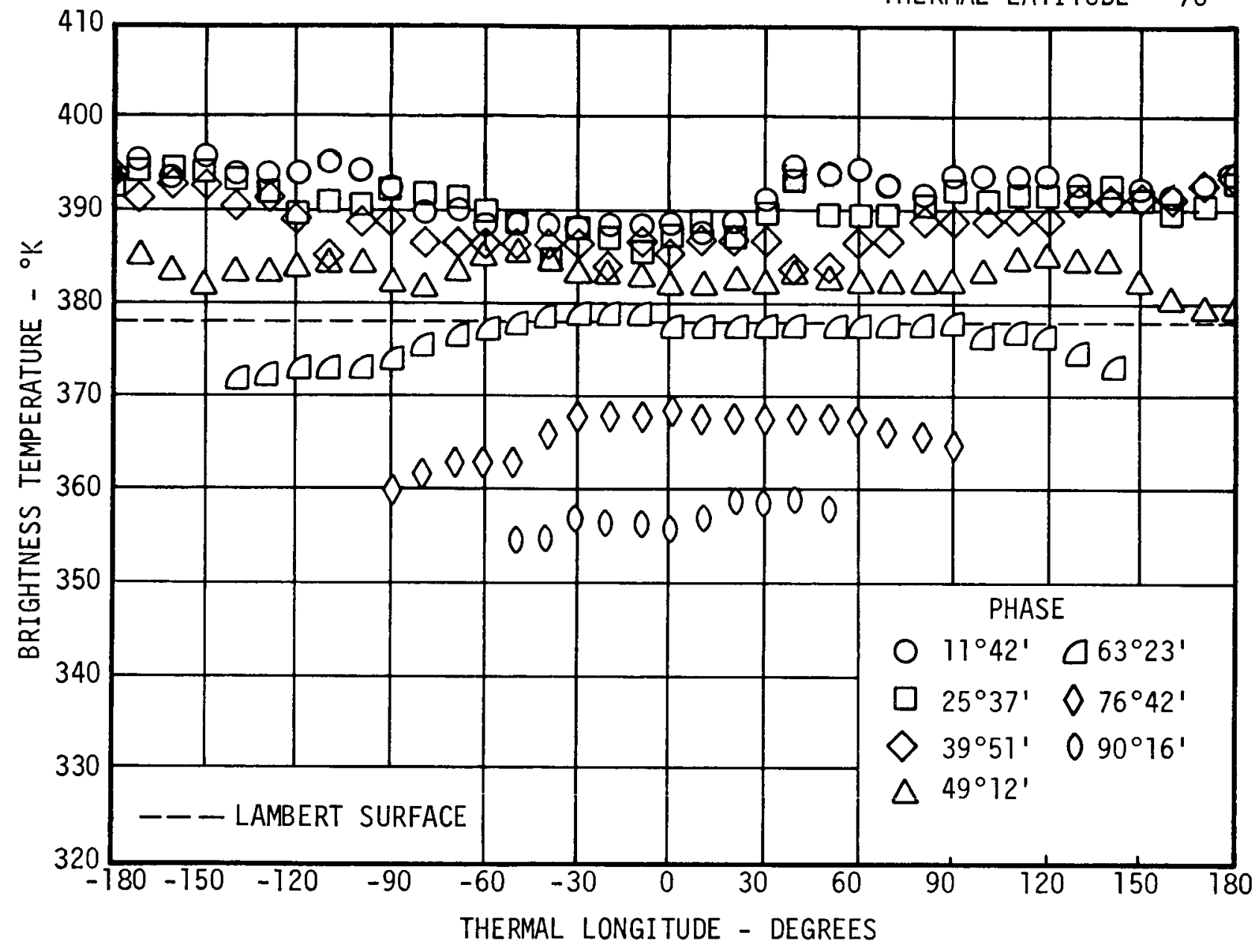

Figure 56. Brightness Temperature Versus Thermal Longitude for Positive Phases at Thermal Latitude $70^{\circ}$ 


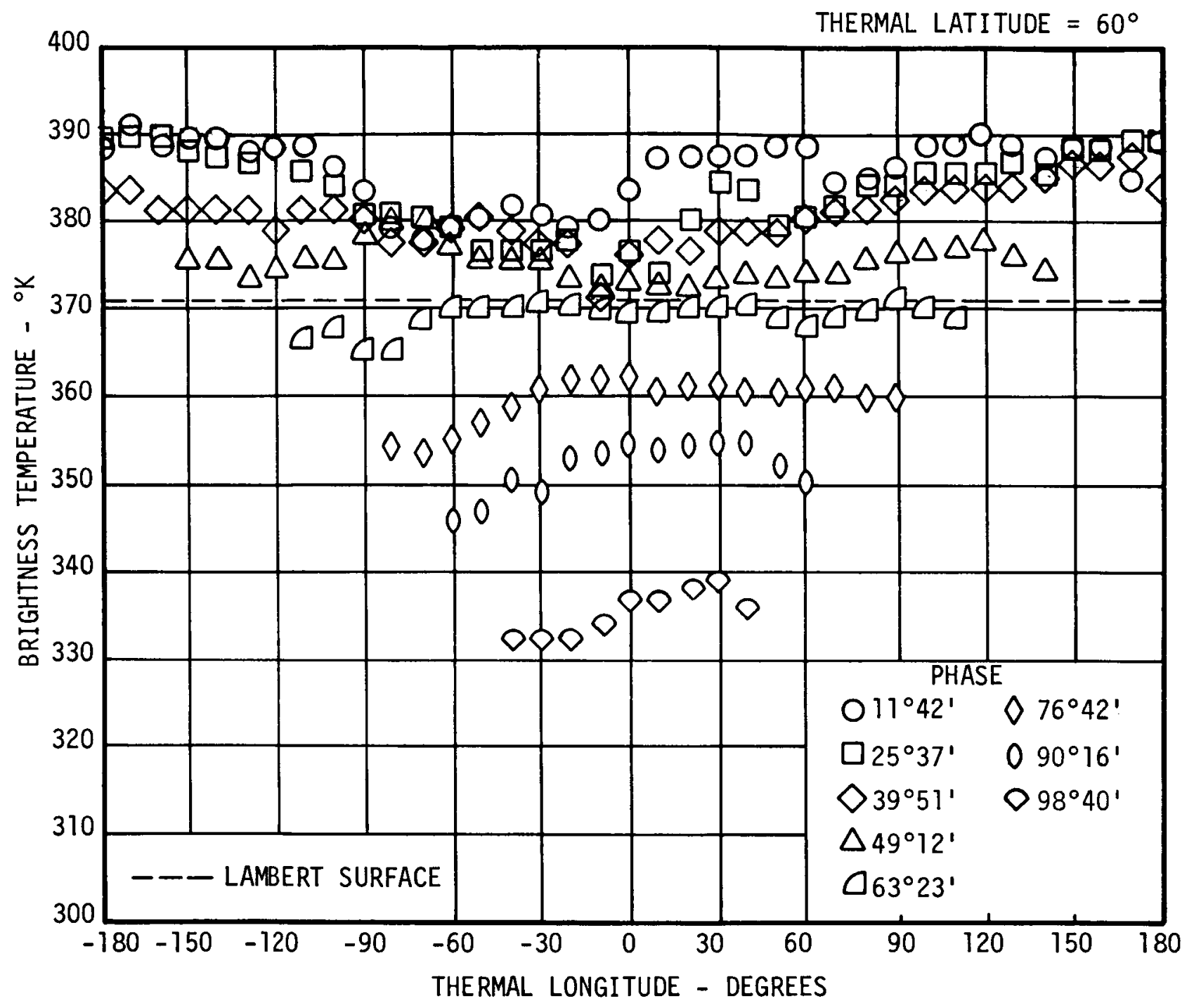

Figure 57. Brightness Temperature Versus Thermal Longitude for Positive Phases at Thermal Latitude $60^{\circ}$ 
THERMAL LATITUDE $=50^{\circ}$

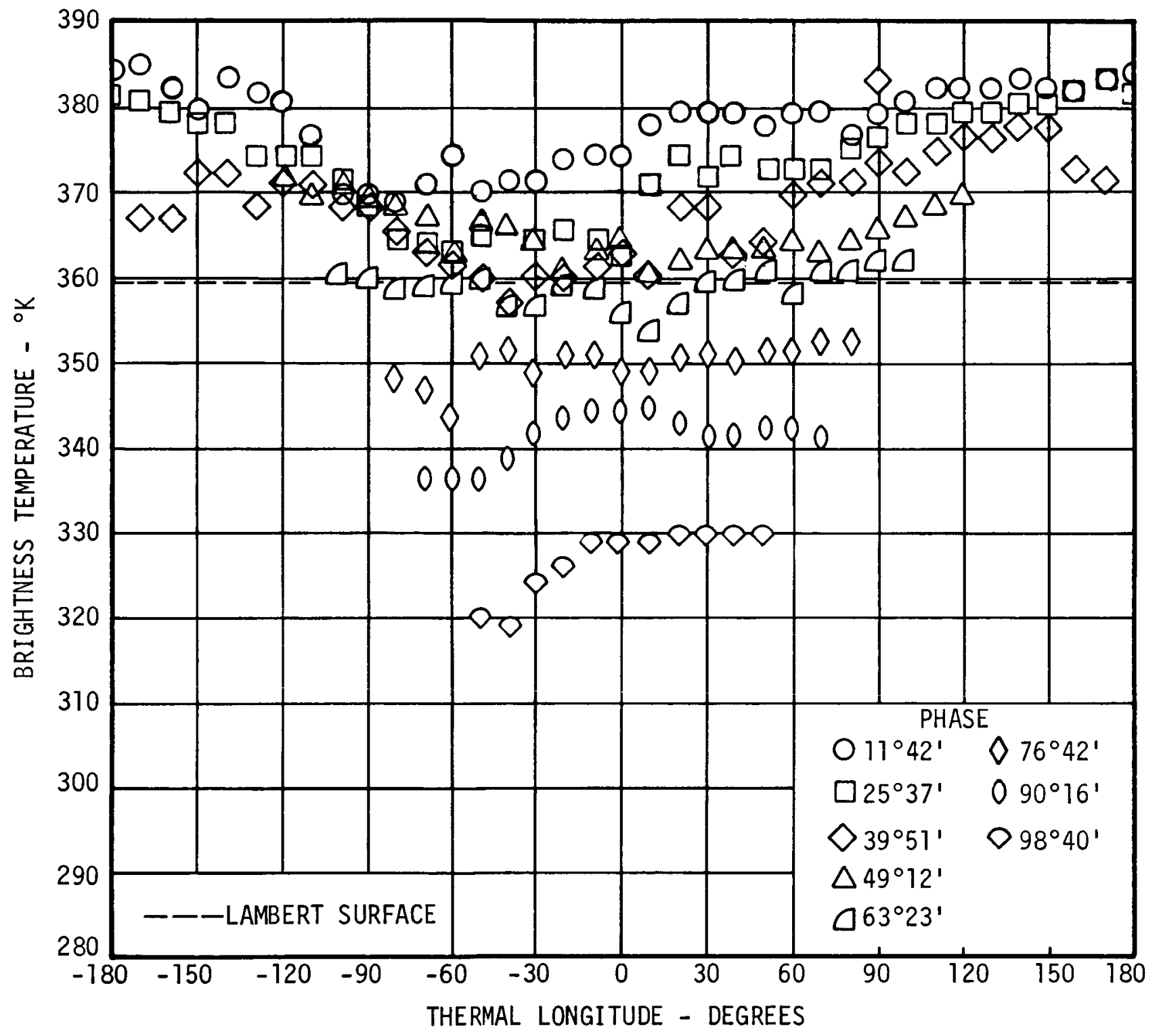

Figure 58. Brightness Temperature Versus Thermal Longitude for Positive Phases at Thermal Latitude $50^{\circ}$ 
THERMAL LATITUDE $=40^{\circ}$

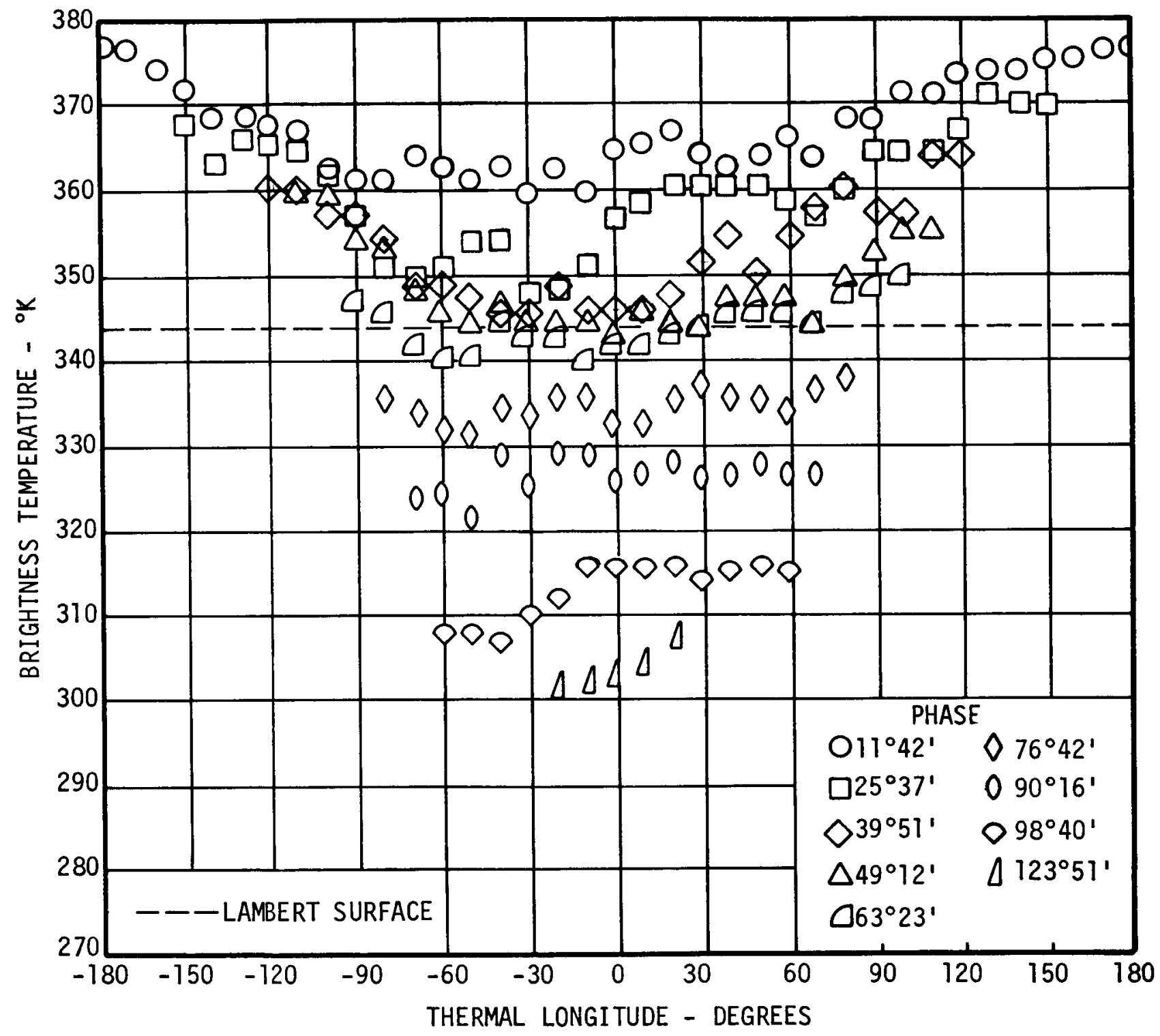

Figure 59. Brightness Temperature Versus Thermal Longitude for Positive Phases at Thermal Latitude $40^{\circ}$ 
THERMAL LATITUDE $=30^{\circ}$

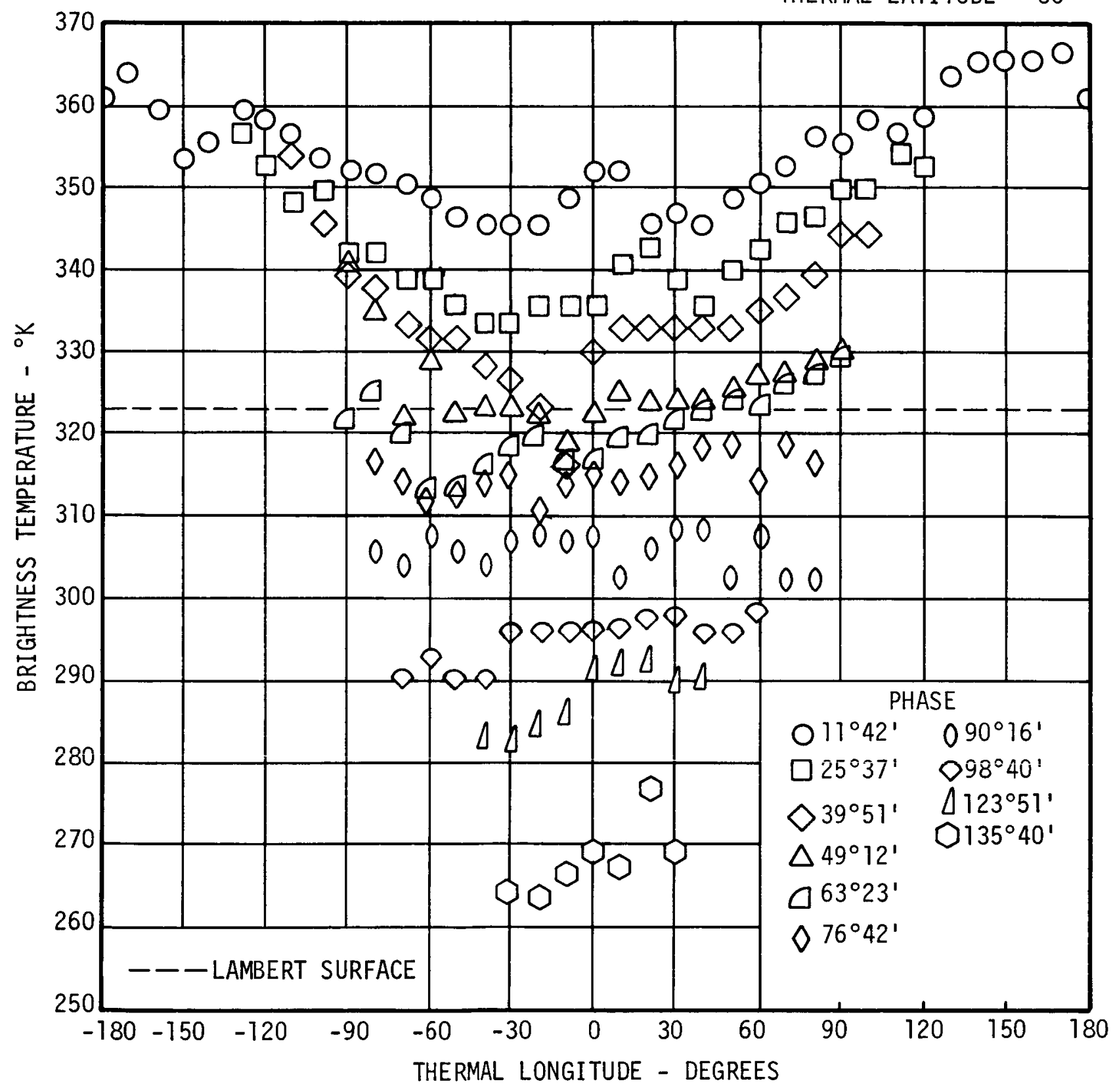

Figure 60. Brightness Temperature Versus Thermal Longitude for Positive Phases at Thermal Latitude $30^{\circ}$ 


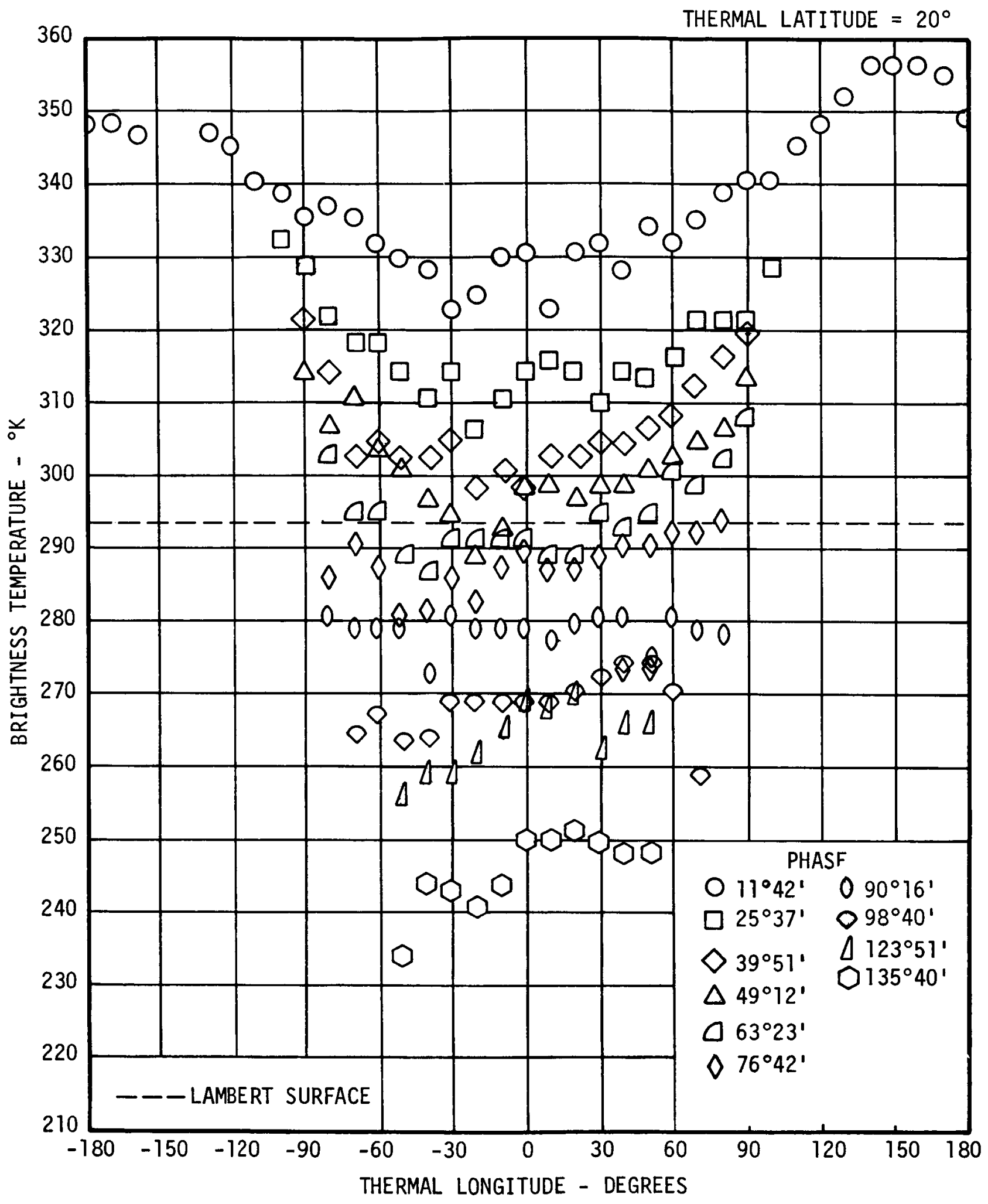

Figure 61. Brightness Temperature Versus Thermal Longitude for Positive Phases at Thermal Latitude $20^{\circ}$ 
THERMAL LATITUDE $=10^{\circ}$

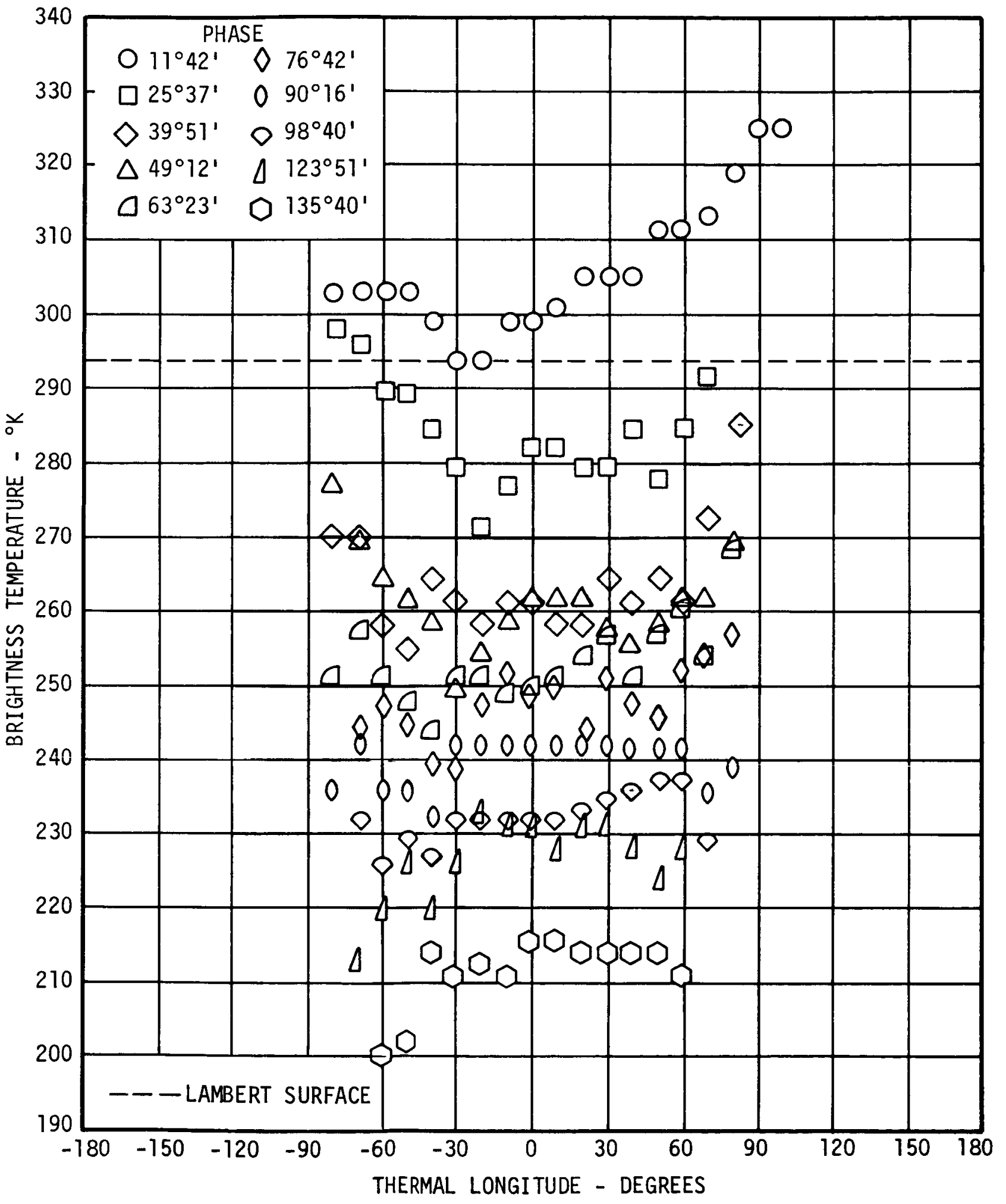

Figure 62. Brightness Temperature Versus Thermal Longitude for Positive Phases at Thermal Latitude $10^{\circ}$ 
THERMAL LATITUDE $=80^{\circ}$

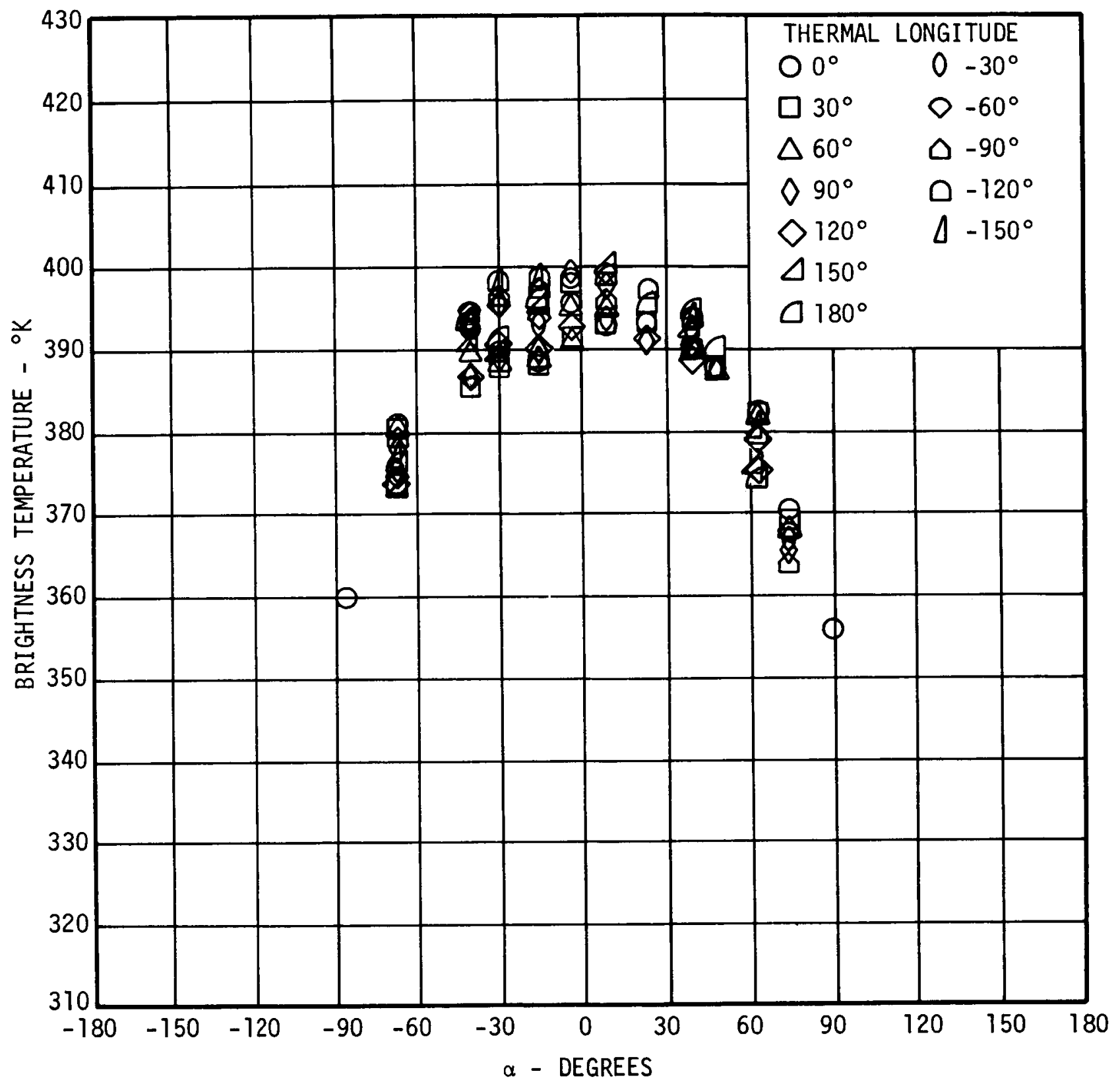

Figure 63. Brightness Temperature Versus Phase at Thermal Latitude $80^{\circ}$ 


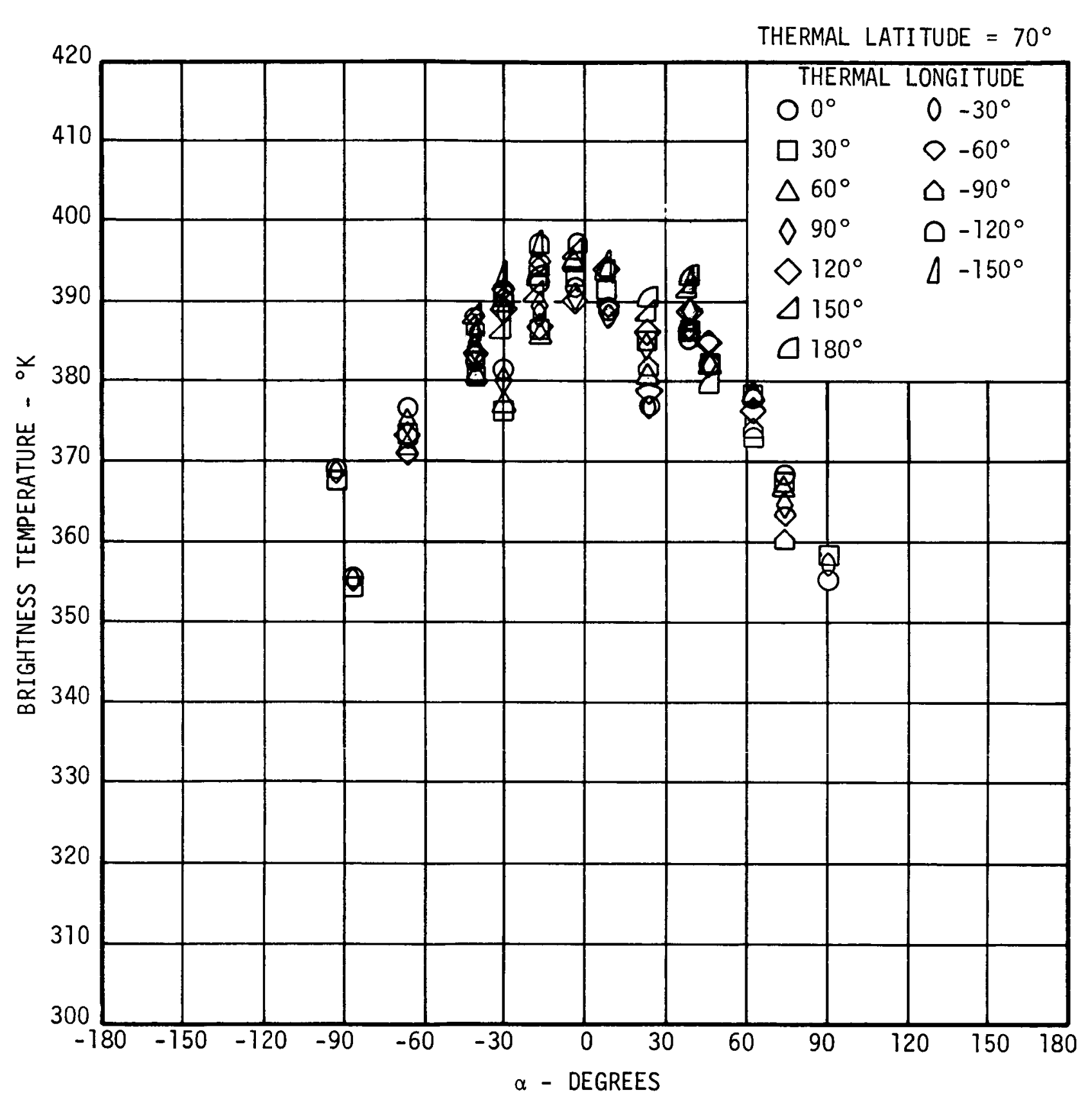

Figure 64. Brightness Temperature Versus Phase at Thermal Latitude $70^{\circ}$ 


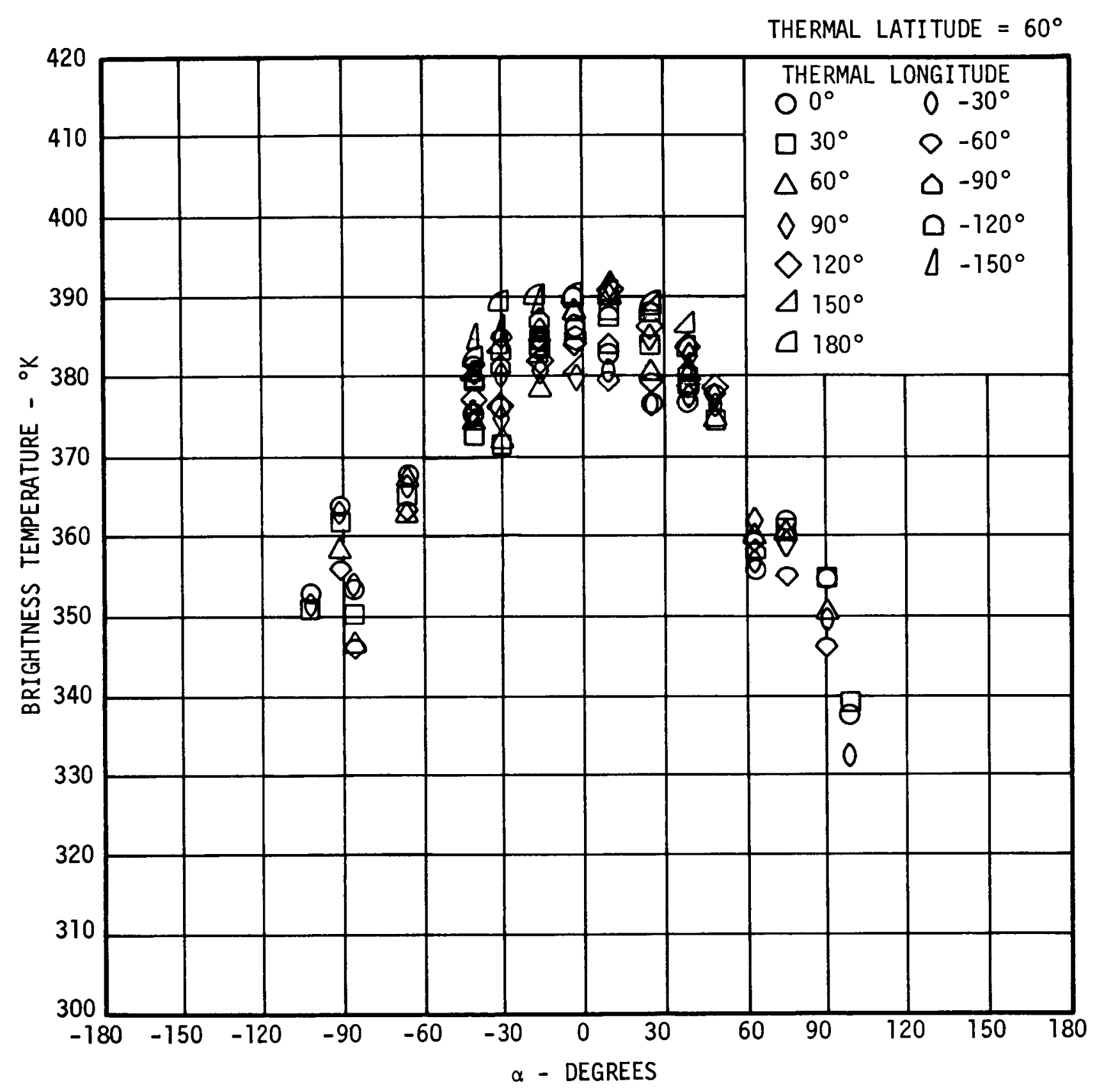

Figure 65. Brightness Temperature Versus Phase at Thermal Latitude $60^{\circ}$ 
THERMAL LATITUDE $=50^{\circ}$

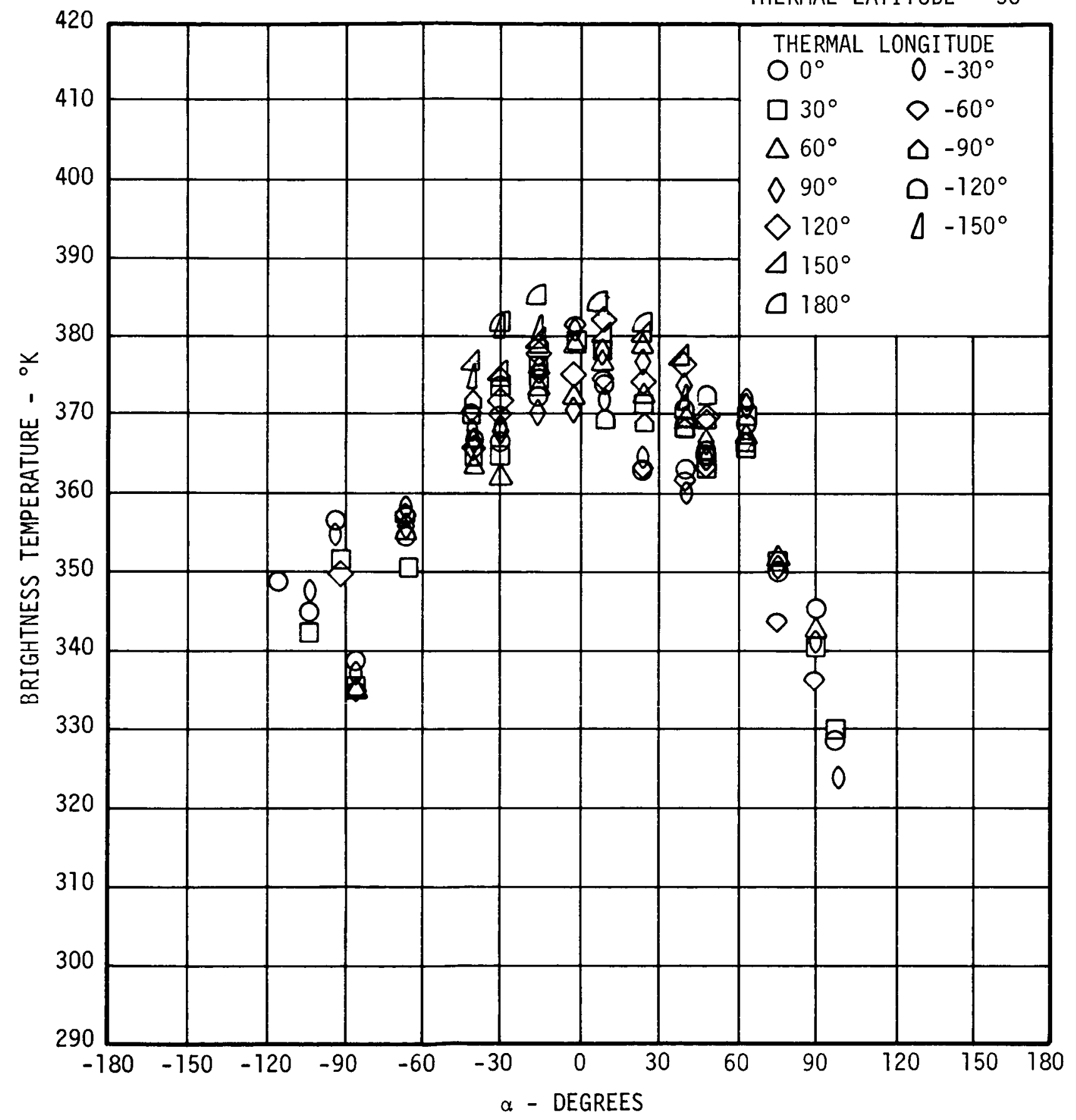

Figure 66. Brightness Temperature Versus Phase at Thermal Latitude $50^{\circ}$ 


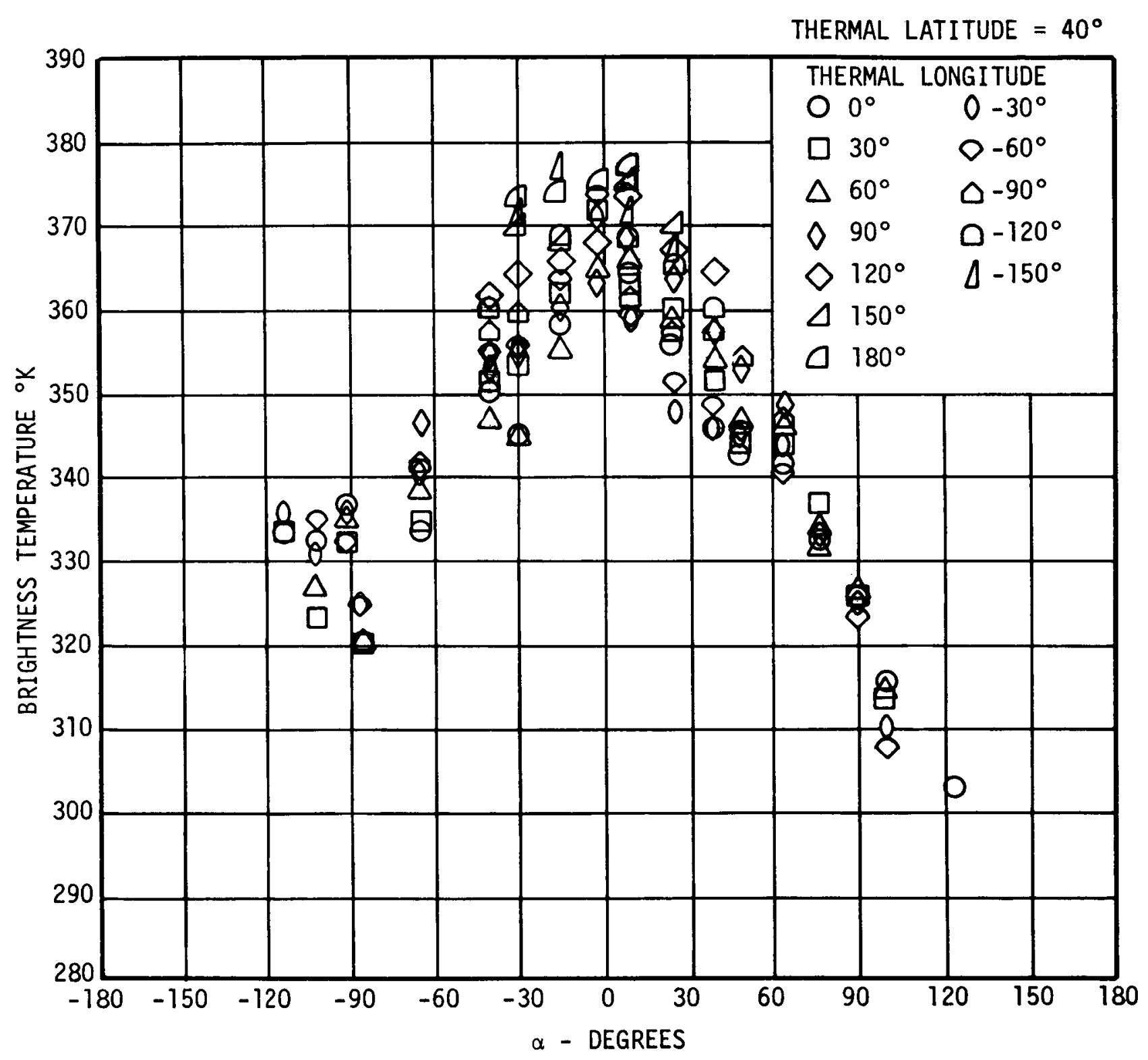

Figure 67. Brightness Temperature Versus Phase at Thermal Latitude $40^{\circ}$ 


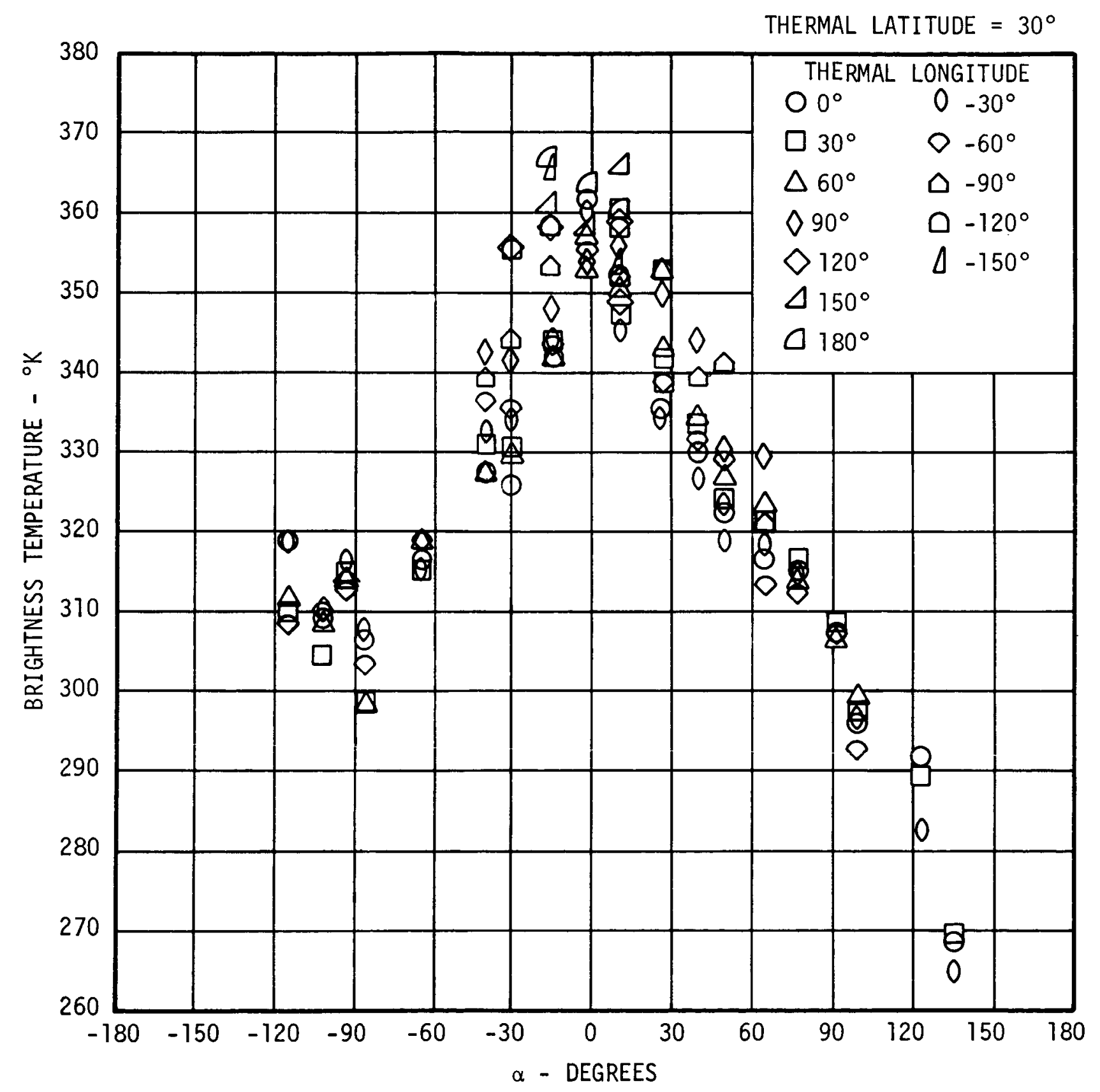

Figure 68. Brightness Temperature Versus Phase at Thermal Latitude $30^{\circ}$ 


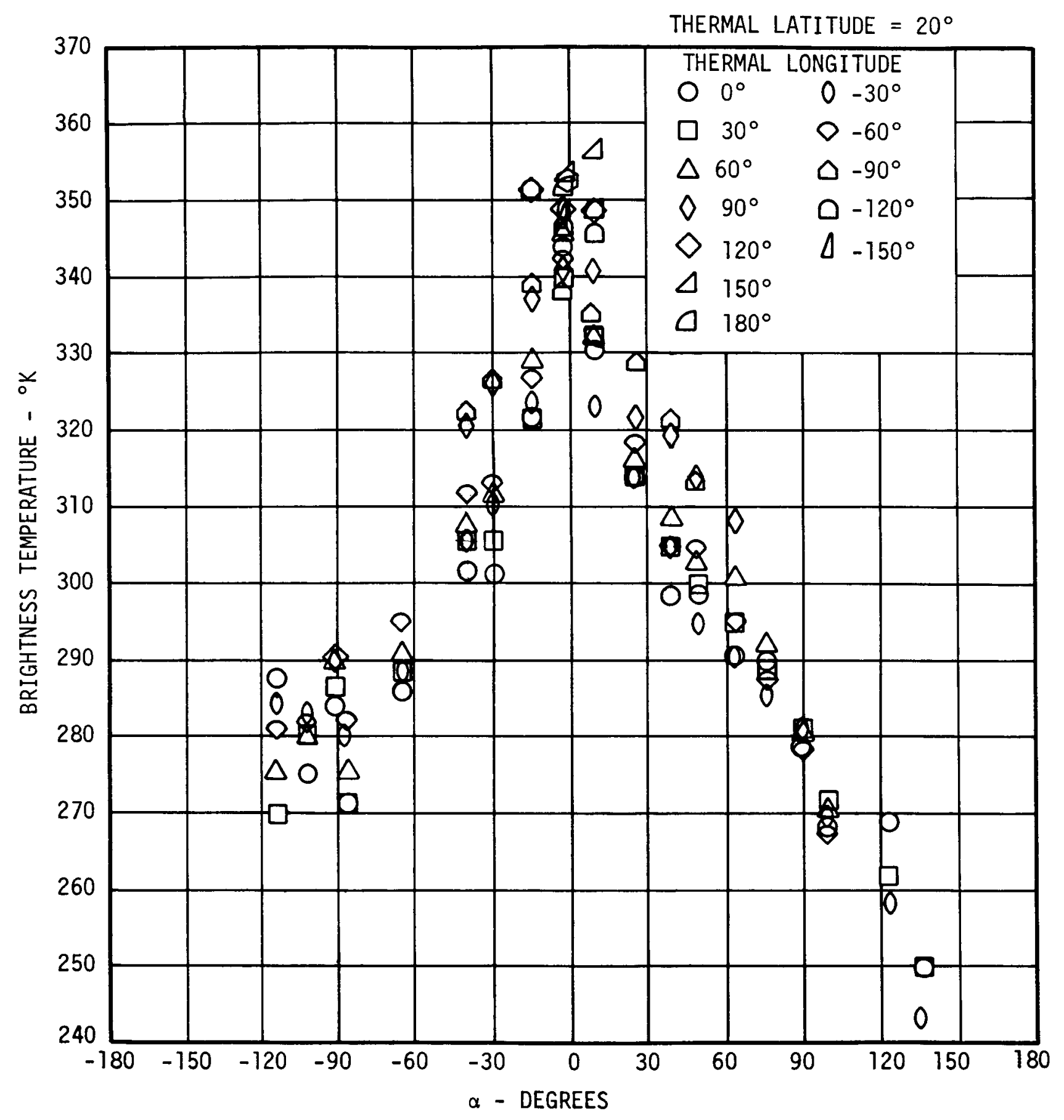

Figure 69. Brightness Temperature Versus Phase at Thermal Latitude $20^{\circ}$ 


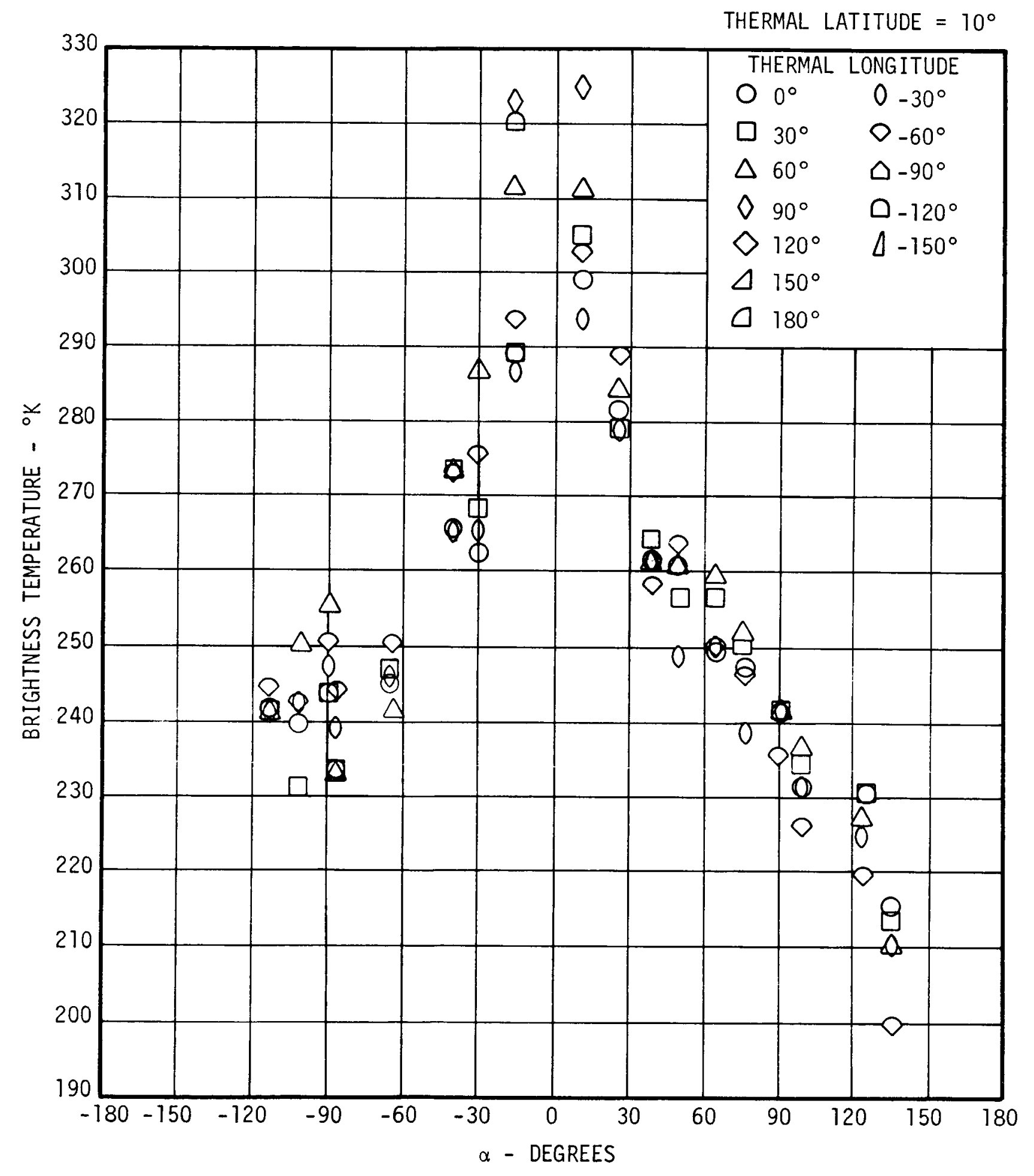

Figure 70. Brightness Temperature Versus Phase at Thermal Latitude $10^{\circ}$ 


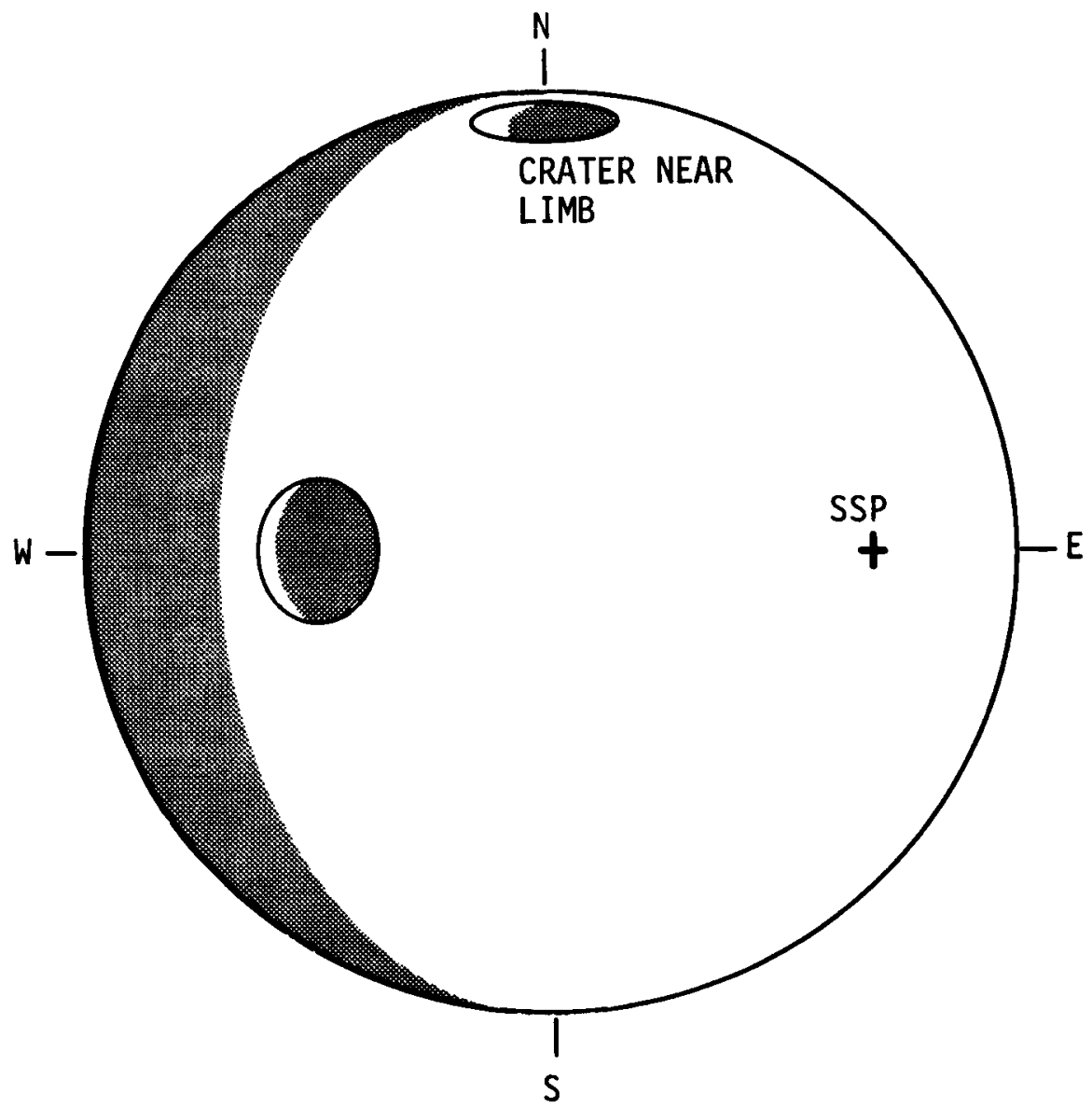

Figure 71. Variation in Ratio of Shadow to Illuminated Area Between Two Craters Equal Angular Distance from the Subsolar Point, as Seen by an EarthSensor 


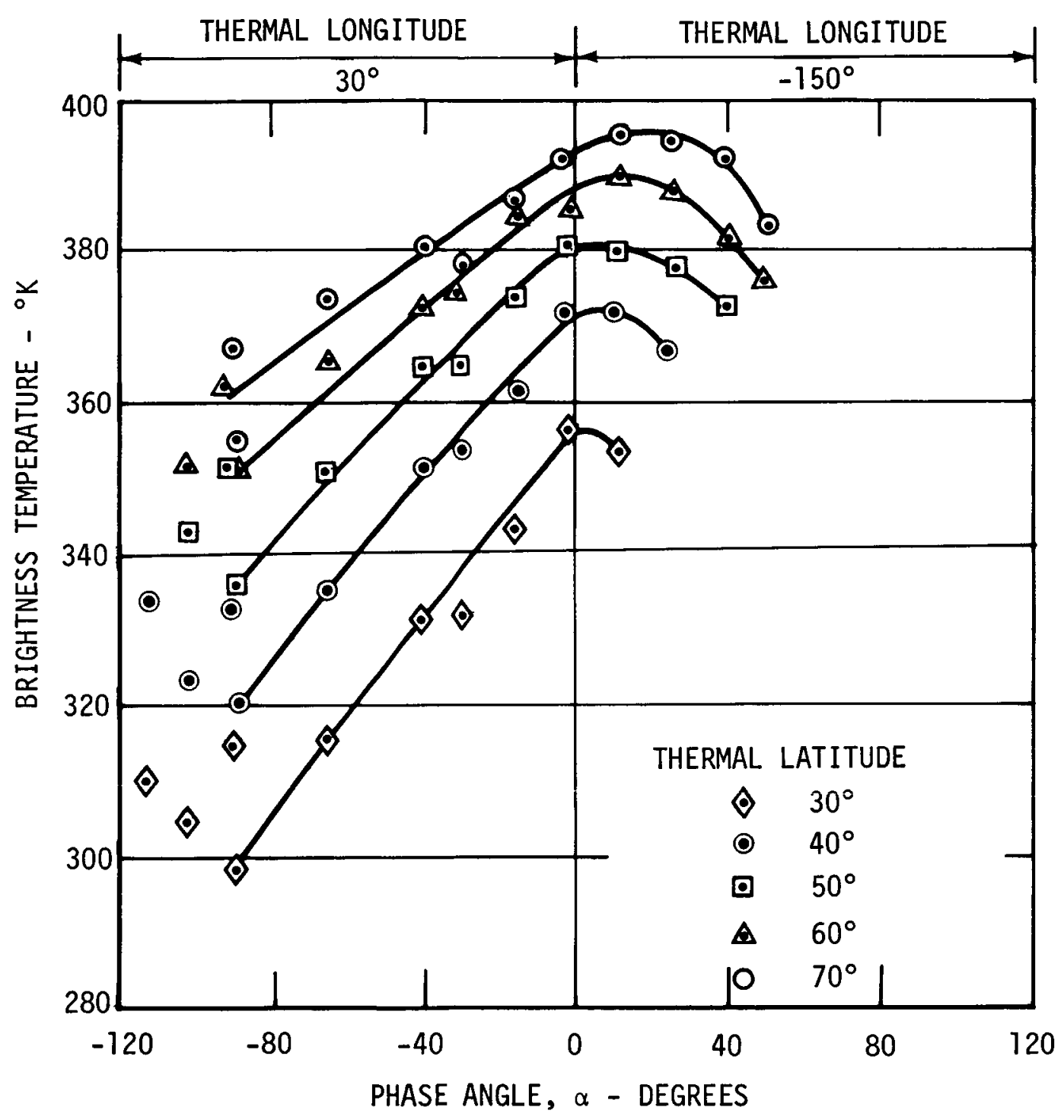

Figure 72. Brightness Temperature Versus Phase for $30^{\circ}$ Thermal Longitude Before Full Moon and for $-150^{\circ}$ Thermal Longitude After Full Moon at Several Thermal Latitudes 


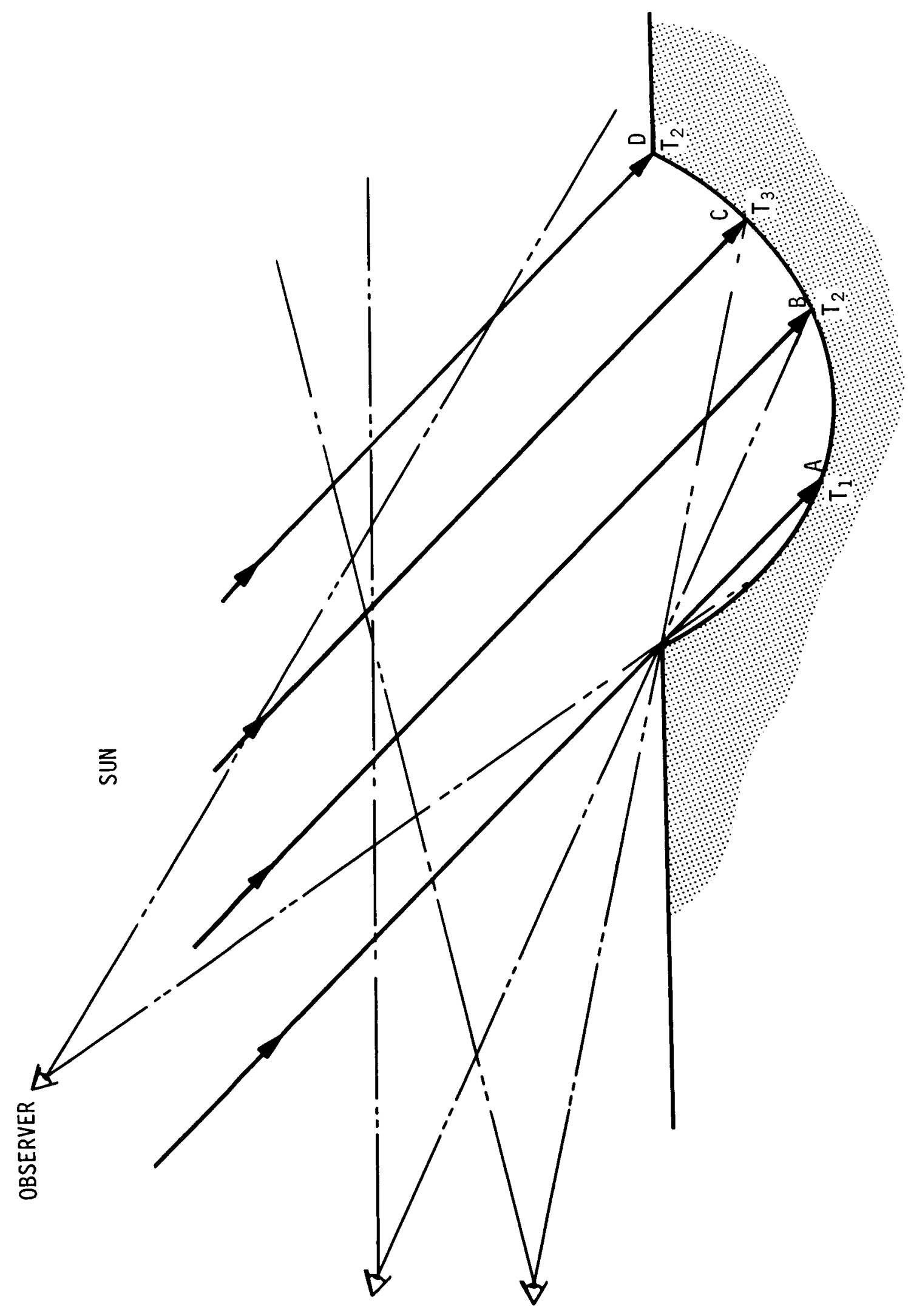

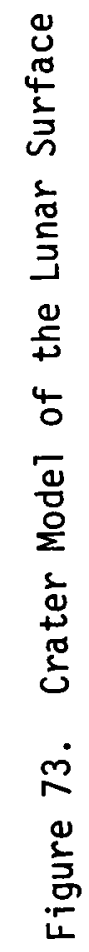




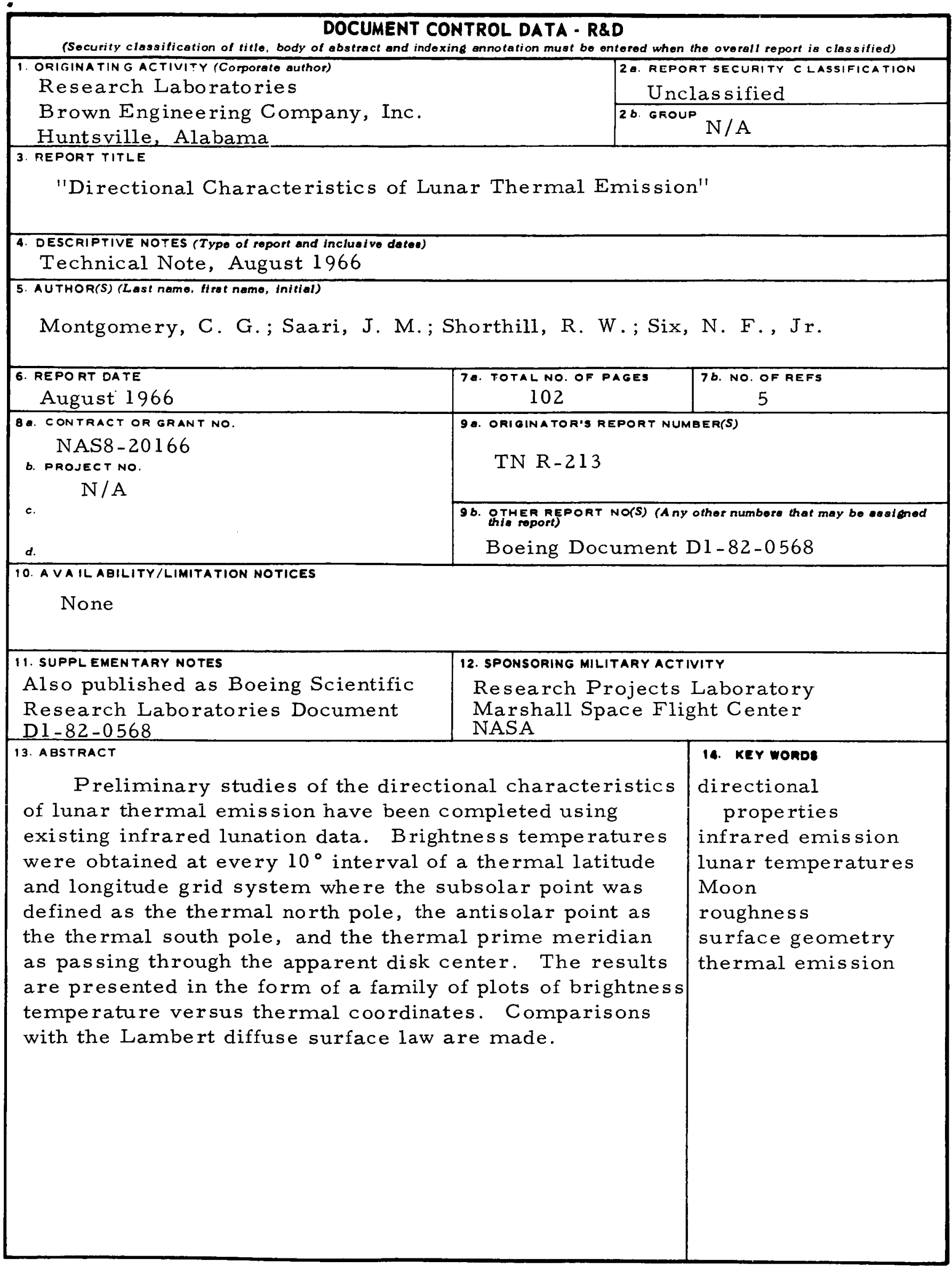

